\title{
Minimum energy multiple crack propagation. Part-II: Discrete Solution with XFEM
}

Danas Sutula ${ }^{\mathrm{a}, \mathrm{b}}$, Pierre Kerfriden ${ }^{\mathrm{b}}$, Tonie van Dam ${ }^{\mathrm{a}}$, and Stéphane P.A. Bordas ${ }^{\mathrm{c}, \mathrm{a}},{ }^{*}$

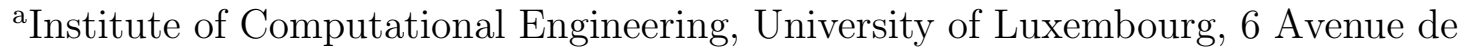
la Fonte, 4362 Esch-sur-Alzette, Luxembourg

bSchool of Engineering, Cardiff University, Queen's Building, The Parade,

Cardiff, CF24 3AA, UK

${ }^{c}$ Visiting Professor, Institute of Research and Development, Duy Tan University,

K7/25 Quang Trung, Danang, Vietnam

*stephane.bordas@alum.northwestern.edu

July 17, 2017

\section{Contents}

\begin{tabular}{lll}
\hline 1 & Introduction & 3
\end{tabular}

$\begin{array}{|lr|}2 & \text { Definitions and assumptions }\end{array}$

\begin{tabular}{llr}
\hline & Discrete solution methods & $\mathbf{8}$
\end{tabular}

3.1 Crack growth by fractured length control . . . . . . . . . . . . . . 8

3.2 Crack growth by external load control . . . . . . . . . . . . . . . . . . . 13

3.3 Energy-gradient based crack growth . . . . . . . . . . . . . . . . . . 14

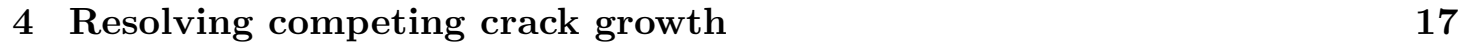

4.1 Detection of crack tip competition . . . . . . . . . . . . . . . . 17

4.2 Determination of critical crack tips . . . . . . . . . . . . . . . . 18

4.3 Summary of solution algorithm . . . . . . . . . . . . . . . . . . 21

4.4 Verification of solution approach $\ldots \ldots \ldots \ldots$. . . . . . . . . . 23

$\begin{array}{llr}5 \text { Discretisation } & \mathbf{2 7}\end{array}$

$5.1 \quad$ Discrete energy release rates . . . . . . . . . . . . . . . . . . . . 27

5.2 Stiffness and force derivatives $\ldots \ldots \ldots$. . . . . . . . . . . . . . . . . 29

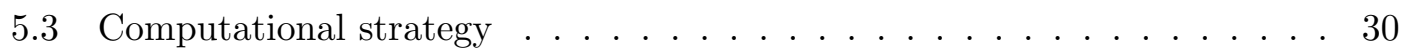

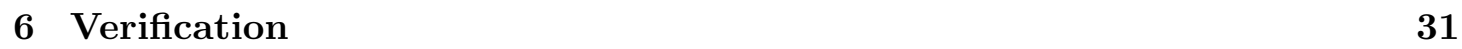


6.1 Rotational energy release rates . . . . . . . . . . . . . . . . . . . . 31

6.2 Comparison to the stress criterion . . . . . . . . . . . . . . . . . . . . . 34

6.3 Conclusion ...................... . . 41

\begin{tabular}{lll}
\hline 7 & Summary & 41
\end{tabular}

\begin{tabular}{lll}
\hline & Supplementary material & 42
\end{tabular}

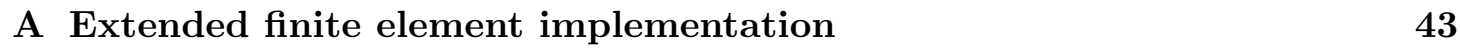

A.1 XFEM approximation . . . . . . . . . . . . . . . . . . . 43

A.2 XFEM equation system . . . . . . . . . . . . . . . . . . . . . . 44

A.3 Element-level derivatives . . . . . . . . . . . . . . . . . . . . . . . . 46

\begin{tabular}{ll}
\hline References & 50
\end{tabular}

\section{Abstract}

The three-part paper deals with energy-minimal multiple crack propagation in a linear elastic solid under quasi-static conditions. The principle of minimum total energy, i.e. the sum of the potential and fracture energies, which stems directly from the Griffith's theory of cracks, is applied to the problem of arbitrary crack growth in 2D. The proposed formulation enables minimisation of the total energy of the mechanical system with respect to the crack extension directions and crack extension lengths to solve for the evolution of the mechanical system over time. The three parts focus, in turn, on (I) the theory of multiple crack growth including competing cracks, (II) the discrete solution by the extended finite element method using the minimum-energy formulation, and (III) the aspects of computer implementation within the Matlab programming language. The Part-II of our three-part paper examines three discrete solution methods for solving fracture mechanics problems based on the principle of minimum total energy. The suitability of each solution approach is determined by the stability property of the fracture configuration at hand. The first method is based on external load-control. It is suitable for stable crack growth and stable fracture configurations. The second method is based on fracture area-control (or length-control in 2D). This method is applicable to stable or unstable fracture growth but the fracture front must be stable. The third solution method is based on a gradient-descent. Although the method is aimed at solving general crack growth problems, its weak point is that the converged solution can not be guaranteed to be optimal in the particular case of competing crack growth and an unstable fracture front configuration. Nonetheless, the main focus is on the implementation and application of the gradient-descent solution approach within the framework of the extended finite element method. Concerning the aforementioned case of competing crack growth, an alternative solution strategy is pursued to supplement the gradient-descent approach. The proposed method, however, is only a proof of concept since its robustness 
is assessed by solving fabricated benchmark problems. The open-source Matlab code, documentation and example cases are included as supplementary material.

\section{Introduction}

Part-I of this three-part paper dealt with the problem of minimum energy multiple crack growth following Griffith's theory of brittle fracture. The main outcomes of Part-I were the derivation of the crack growth direction criterion based on the minimum energy principle and the theoretical analysis of competing crack growth behaviour. Three solution methods were presented for determining competing crack tip growth rates for different cases of fracture front stabilities. The present part, Part-II, of our three-part paper is concerned with the application of the energy minimisation formulations within a discrete framework. We describe three solution methods for the problem of multi-crack growth which we tailor to the stability properties of the fracture configuration. In addition, we propose a method for tackling competing crack growth within a discrete framework that assumes fixed-length crack tip extensions. Although our energy minimisation approaches are implemented within the framework of the extended finite element method (XFEM), the core methodologies are not restricted to XFEM. Indeed, we keep the descriptions of our proposed approaches as general as possible. In particular, the key ingredient to the success of our approach is the stiffness derivative method [23, 20] for computing the crack tip energy release rates. As the method was originally proposed for the finite element method, it is easily generalisable to other mesh-based discretisations and beyond.

Our motivation is to implement a numerical framework for modelling multi-crack growth consistently with Griffith's theory of brittle fracture [15, 16]. To this end, we apply the fundamental principle of energy minimality of the cracked solid $6,29,28$ to determine the evolution of cracks. The commonly used criteria in Linear Elastic Fracture Mechanics (LEFM), such as: maximum hoops stress [11], principle of local symmetry [14], minimum strain energy density [30], are heuristic approximations to Griffith's theory. The maximum energy release rate criterion [19, 21, 18], which implies energy minimality of the cracked solid, is the only criterion that is consistent with Griffith's theory. Even so, the typical numerical implementations of the aforementioned criteria rely on the crack tip stress intensity factors (SIF), which are determined using auxiliary crack tip fields derived from a single-crack problem in an infinite solid [34]. These auxiliary solutions, which are first order accurate, are used within the discrete context regardless of how close the crack tip is to another crack tip or to a boundary of the domain. Furthermore, a closed form relationship between the crack tip SIF and the energy release rate in an arbitrary crack growth direction is not available to the best of our knowledge. The 2004 paper [5] that deals with multi-crack growth within XFEM, relies on crack tip SIF and on the maximum-hoop stress criterion to determine the crack growth direction.

Our approach, in contrast, does not rely on crack tip SIF or on any closed form solution in order to determine the crack growth direction. Instead, the fundamental concern is the 
minimisation of the total energy functional to determine the evolution of cracks.

The outline of this paper is as follows. Section 2 gives a summary of the relationships between the stability of the fracture configuration and the shape of the energy function. In addition, the expressions of the energy release rates are recalled. Section 3 describes three solution methods to the problem of multi-crack growth within a discrete framework; these methods are based namely on: fracture-area (or length in 2D) control, load-control, and on the energy gradient. Section 4 proposes a method for resolving competing crack growth as well as verifies it against different cases of competing crack growth. Section 5 describes the discrete implementation of the algebraic computations of the energy release rates within XFEM. Section 6 verifies the accuracy of the computed energy release rates and then goes on to compare the minimum energy criterion to the classic maximum hoop stress criterion. Section 8 provides some conclusions and sets the stage for Part-III.

\section{Definitions and assumptions}

The evolution of cracks is governed by the time-continuous minimisation of the total energy functional $\mathcal{E}(\mathbf{u}(\ell), \boldsymbol{\theta}(\ell), \ell)$ with respect to the growing crack tip branch lengths $\boldsymbol{\ell}=\left\{\ell_{i}\right\}_{i \in \mathcal{I}_{\text {tip }}}$ where $\mathcal{I}_{\text {tip }}=\left\{1,2, \ldots, n_{\text {tip }}\right\}$ is the set of all crack tips. The displacement field $\mathbf{u}(\ell)$ and the branch angular orientations $\boldsymbol{\theta}(\boldsymbol{\ell})=\left\{\theta_{i}\left(\ell_{i}\right)\right\}_{i \in \mathcal{I}_{\text {tip }}}$ evolve together with $\boldsymbol{\ell}$ such that $\mathcal{E}(\ell)$ is always minimised. The derivation of this functional was presented in Part-I (Sections 5 and 6 ). In summary, the general form can be written as:

$$
\mathcal{E}(\mathbf{u}(\ell), \boldsymbol{\theta}(\ell), \ell)=\Pi(\mathbf{u}(\ell), \boldsymbol{\theta}(\ell), \ell)+\sum_{i=1}^{n_{\text {tip }}} \int_{\ell_{i}} G_{\mathrm{ci}} \mathrm{d} l_{i}
$$

where $\Pi$ is the linear-elastic potential energy of the cracked solid, $G_{\mathrm{c} i}$ is the critical fracture energy of the material at crack tip $i$, and $l_{i}$ is a dummy variable for a crack tip branch length. Although $G_{\mathrm{c} i}$ can generally depend on the fracture path (e.g. if the material is anisotropic or if it can be characterised by a non-constant fracture resistance) we will assume for simplicity a homogeneous material with a constant $G_{\mathrm{c}}$. Moreover, we will not consider the possibility of crack surface contact or compressive crack growth; instead, our focus will be on fracture problems that are driven by tensile loading.

Within a discrete 2D framework that assumes straight finite-length crack tip extensions at sharp kink angles, the evolution of the displacement field $\mathbf{u}$, crack tip branch lengths $\boldsymbol{\ell}$ and their angular orientations $\boldsymbol{\theta}$ can be given as a sequence of solutions of the variable triplet $\{\mathbf{u}, \boldsymbol{\ell}, \boldsymbol{\theta}\}\left(a^{k}\right)$ where $a^{k}=a_{0}+k \Delta a$ is the total fractured length at a discrete time $k \in\{1,2, \ldots\}$ and where $\Delta a$ is the total fracture length increment per time-step $k$.

Since the discrete solution needs to be obtained incrementally, the minimisation of the total energy at the $k^{\prime}$ 'th time-step $\mathcal{E}^{k}=\mathcal{E}\left(\mathbf{u}^{k}, \boldsymbol{\ell}^{k-1}+\Delta \boldsymbol{\ell}^{k}, \boldsymbol{\theta}^{k-1}+\Delta \boldsymbol{\theta}^{k}\right)$ can be carried out 
with respect to the variable triplet $\{\mathbf{u}, \Delta \boldsymbol{\ell}, \Delta \boldsymbol{\theta}\}^{k}$, where $\Delta \boldsymbol{\ell}^{k}$ are the crack tip extension lengths and $\Delta \boldsymbol{\theta}^{k}$ are the crack tip kink angles. Generally, an iterative solution approach needs to be used to minimise $\mathcal{E}^{k}$ because the variables $\{\mathbf{u}, \Delta \boldsymbol{\ell}, \Delta \boldsymbol{\theta}\}^{k}$ are non-linearly coupled. Furthermore, the sought solution needs to satisfy two constraint conditions: (1) crack growth irreversibility: $\forall i \in \mathcal{I}_{\text {tip }} \Delta \ell_{i}^{k} \geq 0$, and (2) a prescribed amount of total fractured length increase: $\sum_{i \in \mathcal{I}_{\text {tip }}} \Delta \ell_{i}^{k}=\Delta a$.

We will outline three solution strategies where the choice of a particular strategy will be affected by the stability of the fracture configuration at hand. We can recall from Part-I of this three-part paper that the idea of fracture stability simply provides a way of characterising the shape of the total energy function with respect to the crack tip branch perturbations $\delta \boldsymbol{\ell}$. Specifically, we classify fracture front stability as follows:

$$
\begin{array}{cll}
\text { stable fracture front : } & \forall \delta \boldsymbol{\ell} \in\left\{\boldsymbol{l} \in \mathbb{R}^{n_{\mathrm{tip}}}: \sum_{i=1}^{n_{\mathrm{tip}}} \delta l_{i}=0\right\} & \frac{\partial^{2} \mathcal{E}}{\partial \ell_{i} \partial \ell_{j}} \delta \ell_{i} \delta \ell_{j}>0 \\
\text { unstable fracture front : } & \forall \delta \boldsymbol{\ell} \in\left\{\boldsymbol{l} \in \mathbb{R}^{n_{\mathrm{tip}}}: \sum_{i=1}^{n_{\mathrm{tip}}} \delta l_{i}=0\right\} & \frac{\partial^{2} \mathcal{E}}{\partial \ell_{i} \partial \ell_{j}} \delta \ell_{i} \delta \ell_{j} \leq 0 \\
\text { partially stable front : } & \exists \delta \boldsymbol{\ell} \in\left\{\boldsymbol{l} \in \mathbb{R}^{n_{\mathrm{tip}}}: \sum_{i=1}^{n_{\mathrm{tip}}} \delta l_{i}=0\right\} & \frac{\partial^{2} \mathcal{E}}{\partial \ell_{i} \partial \ell_{j}} \delta \ell_{i} \delta \ell_{j} \geq 0
\end{array}
$$

A stable fracture front (2) means the energy function curves up with respect to any admissible perturbation of the crack tip branch lengths. An unstable front (3) means the energy function curves down. A partially stable front (4) means the energy function curves up or down - depending on the perturbation. The admissible perturbations are positive at some tips and negative at others such that the net change in the total length of the crack tip branches is zero. Similarly we classify the fracture growth stability:

$$
\begin{array}{rll}
\text { stable fracture growth : } & \forall \delta \boldsymbol{\ell} \in\left\{\boldsymbol{l} \in \mathbb{R}^{n_{\text {tip }}}: \sum_{i=1}^{n_{\text {tip }}} \delta l_{i}=\Delta a\right\} & \frac{\partial^{2} \mathcal{E}}{\partial \ell_{i} \partial \ell_{j}} \delta \ell_{i} \delta \ell_{j}>0 \\
\text { unstable fracture growth : } & \forall \delta \boldsymbol{\ell} \in\left\{\boldsymbol{l} \in \mathbb{R}^{n_{\text {tip }}}: \sum_{i=1}^{n_{\text {tip }}} \delta l_{i}=\Delta a\right\} & \frac{\partial^{2} \mathcal{E}}{\partial \ell_{i} \partial \ell_{j}} \delta \ell_{i} \delta \ell_{j} \leq 0
\end{array}
$$

Stable fracture growth occurs when the energy function curves up with the extension of the crack tips. An unstable growth occurs when the energy function curves down. Figure 1 shows examples of these classifications and their relationship to the behaviour of the energy function. The reader can also refer to Part-I Section 7.1 for more details.

Depending on the stability conditions of the fracture configuration, there are three solution strategies that can be used to solve the discrete fracture growth problem:

1. load-control: for stable fracture front and stable fracture growth

2. length-control: for stable fracture front and (un)stable fracture growth

3. gradient-descent: for (un)stable fracture front and (un)stable fracture growth 


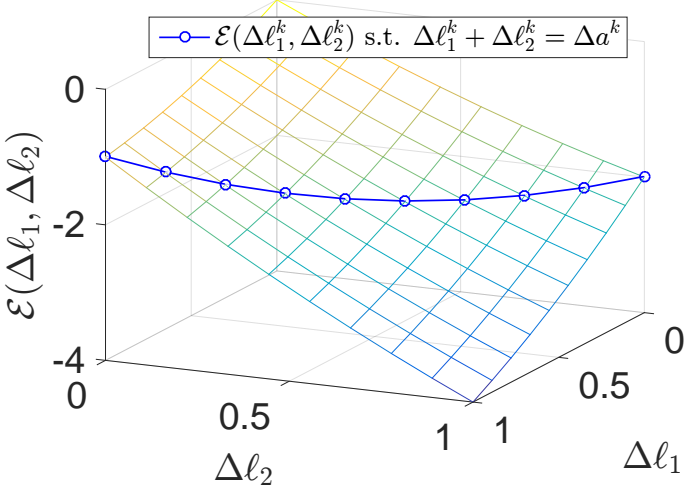

(a) front is stable, growth is stable

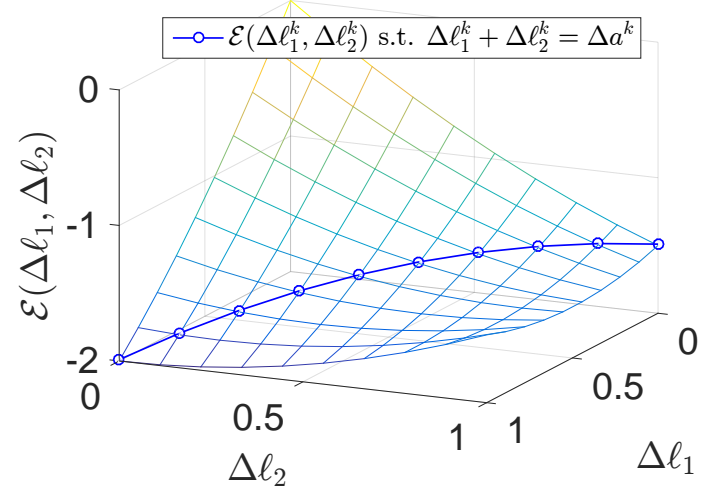

(c) front is unstable, growth is stable

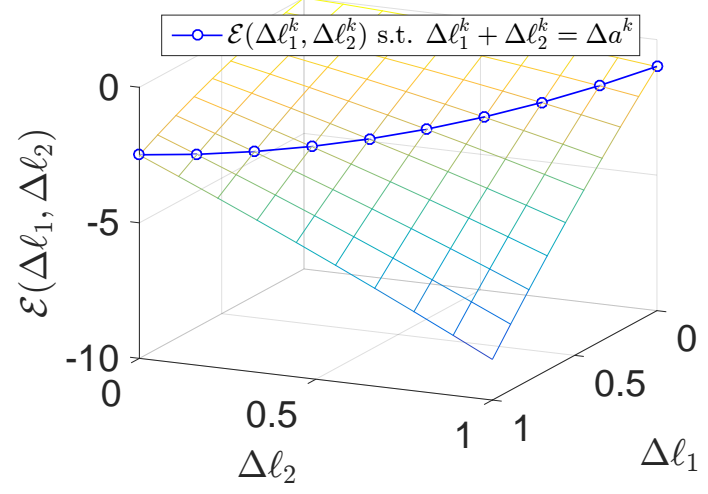

(b) front is stable, growth is unstable

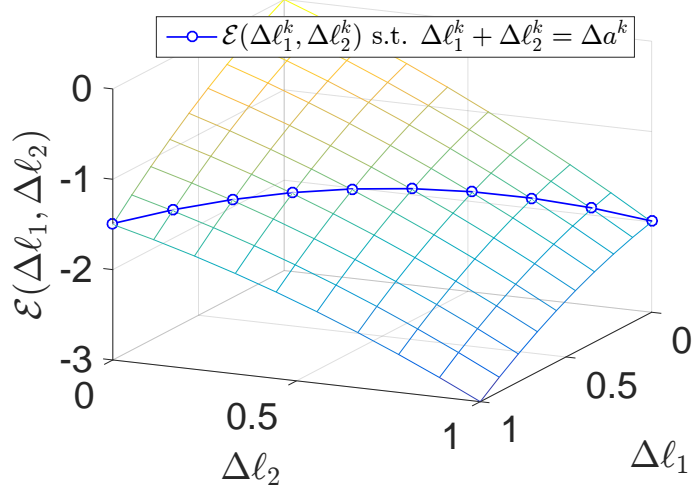

(d) front is unstable, growth is unstable

Figure 1: Hypothetical example cases of possible behaviours of the total energy function (1) considering the extension of two crack tip branches $\ell_{1}$ and $\ell_{2}$. The solid line with circle markers emphasises the behaviour of the energy function subject to the constraint of a fixed amount of total extension of the crack tip branches, i.e. $\Delta \ell_{1}+\Delta \ell_{2}=\Delta a$. 
The technique based on load-control is limited to problems where the energy function $\mathcal{E}^{k}$ is convex (inequalities (2) and (5) hold). On the other hand, the scheme based on the fracture length-control relaxes this requirement insofar that $\mathcal{E}^{k}$ only needs to be convex within the admissible solution space (inequality (2) holds). Finally, the gradient-descent solution approach can be applied to general fracture growth problems where $\mathcal{E}^{k}$ can be non-convex (inequality (4) holds); however, the limitation is that a converged solution can not be guaranteed to be globally optimal (see, for example, Figure 3 ).

The expressions for the energy release rates of finite-length crack tip extensions will be summarised below. Since $G_{\mathrm{c}}$ is assumed to be constant, the derivatives of the total energy function reduce to those of the potential energy function. Firstly, the fracture extension force and the rotational energy release rate are respectively defined as:

$$
\begin{aligned}
G_{\mathrm{s} i} & =-\frac{\partial \Pi(\mathbf{u}, \Delta \boldsymbol{\ell}, \Delta \boldsymbol{\theta})}{\partial \Delta \ell_{i}} \\
G_{\theta i} & =-\frac{\partial \Pi(\mathbf{u}, \Delta \boldsymbol{\ell}, \Delta \boldsymbol{\theta})}{\partial \Delta \theta_{i}}
\end{aligned}
$$

where $i \in \mathcal{I}_{\text {tip }}$ is the crack tip. Similarly, the rates of the energy release rates are:

$$
\begin{gathered}
H_{\mathrm{s} i j}=-\frac{\partial^{2} \mathcal{E}(\mathbf{u}(\Delta \boldsymbol{\ell}, \Delta \boldsymbol{\theta}), \Delta \boldsymbol{\ell}, \Delta \boldsymbol{\theta})}{\mathrm{d} \Delta \ell_{i} \partial \Delta \ell_{j}}=-\frac{\partial^{2} \Pi(\mathbf{u}(\Delta \boldsymbol{\ell}, \Delta \boldsymbol{\theta}), \Delta \boldsymbol{\ell}, \Delta \boldsymbol{\theta})}{\mathrm{d} \Delta \ell_{i} \partial \Delta \ell_{j}} \\
H_{\theta i j}=-\frac{\partial^{2} \mathcal{E}(\mathbf{u}(\Delta \boldsymbol{\ell}, \Delta \boldsymbol{\theta}), \Delta \boldsymbol{\ell}, \Delta \boldsymbol{\theta})}{\mathrm{d} \Delta \theta_{i} \partial \Delta \theta_{j}}=-\frac{\partial^{2} \Pi(\mathbf{u}(\Delta \boldsymbol{\ell}, \Delta \boldsymbol{\theta}), \Delta \boldsymbol{\ell}, \Delta \boldsymbol{\theta})}{\mathrm{d} \Delta \theta_{i} \partial \Delta \theta_{j}} \\
H_{\mathrm{m} i j}=-\frac{\partial^{2} \mathcal{E}(\mathbf{u}(\Delta \boldsymbol{\ell}, \Delta \boldsymbol{\theta}), \Delta \boldsymbol{\ell}, \Delta \boldsymbol{\theta})}{\mathrm{d} \Delta \ell_{i} \partial \Delta \theta_{j}}=-\frac{\partial^{2} \Pi(\mathbf{u}(\Delta \boldsymbol{\ell}, \Delta \boldsymbol{\theta}), \Delta \boldsymbol{\ell}, \Delta \boldsymbol{\theta})}{\mathrm{d} \Delta \ell_{i} \partial \Delta \theta_{j}}
\end{gathered}
$$

The second derivative in the higher order rate terms (9)-(11) is a total derivative; hence, the displacement field needs to be differentiated supposing that static equilibrium holds. Thus, the rate of the energy release rate $H_{\mathrm{s} i j} \equiv \frac{\mathrm{d} G_{\mathrm{s} i}}{\mathrm{~d} \Delta \ell_{j}}$ can also be written as:

$$
\begin{aligned}
& H_{\mathrm{s} i j}=\frac{\mathrm{d}}{\mathrm{d} \Delta \ell_{j}}\left(-\frac{\partial \Pi(\mathbf{u}(\Delta \boldsymbol{\ell}, \Delta \boldsymbol{\theta}), \Delta \boldsymbol{\ell}, \Delta \boldsymbol{\theta})}{\partial \Delta \ell_{i}}\right) \\
& H_{\mathrm{s} i j}=-\left.\frac{\partial^{2} \Pi}{\partial \Delta \ell_{i} \partial \Delta \ell_{j}}\right|_{\mathbf{u}}+\frac{\partial}{\partial \Delta \ell_{i}}\left(\frac{\partial \Pi}{\partial \mathbf{u}}\right)^{\mathrm{T}}\left(\frac{\partial^{2} \Pi}{\partial \mathbf{u}^{2}}\right)^{-1} \frac{\partial}{\partial \Delta \ell_{j}}\left(\frac{\partial \Pi}{\partial \mathbf{u}}\right)
\end{aligned}
$$

Analogous expressions hold for both $H_{\theta i j} \equiv \frac{\mathrm{d} G_{\theta i}}{\mathrm{~d} \Delta \theta_{j}}$ and $H_{\mathrm{m} i j} \equiv \frac{\mathrm{d} G_{\mathrm{s} i}}{\mathrm{~d} \Delta \theta_{j}} \equiv \frac{\mathrm{d} G_{\theta j}}{\mathrm{~d} \Delta \ell_{i}}$ : 


$$
\begin{gathered}
H_{\theta i j}=-\left.\frac{\partial^{2} \Pi}{\partial \Delta \theta_{i} \partial \Delta \theta_{j}}\right|_{\mathbf{u}}+\frac{\partial}{\partial \Delta \theta_{i}}\left(\frac{\partial \Pi}{\partial \mathbf{u}}\right)^{\mathrm{T}}\left(\frac{\partial^{2} \Pi}{\partial \mathbf{u}^{2}}\right)^{-1} \frac{\partial}{\partial \Delta \theta_{j}}\left(\frac{\partial \Pi}{\partial \mathbf{u}}\right) \\
H_{\mathrm{m} i j}=-\left.\frac{\partial^{2} \Pi}{\partial \Delta \ell_{i} \partial \Delta \theta_{j}}\right|_{\mathbf{u}}+\frac{\partial}{\partial \Delta \ell_{i}}\left(\frac{\partial \Pi}{\partial \mathbf{u}}\right)^{\mathrm{T}}\left(\frac{\partial^{2} \Pi}{\partial \mathbf{u}^{2}}\right)^{-1} \frac{\partial}{\partial \Delta \theta_{j}}\left(\frac{\partial \Pi}{\partial \mathbf{u}}\right)
\end{gathered}
$$

Note that a constant external load needs to be supposed in the computations of $H_{\mathrm{s}}, H_{\theta}$ and $H_{\mathrm{m}}$. The load is adjusted (e.g. by scaling it) only after each discrete time increment. The scaling factor is determined such that the crack tip energy release rates are physical, i.e. $G_{\mathrm{s} i} \leq G_{\mathrm{c}} \forall i \in \mathcal{I}_{\text {tip }}$. This is discussed in more detail in Part-I Section 6.2.

\section{Discrete solution methods}

Three solution methods are presented with the aim of achieving energy-minimal fracture propagations. The first strategy assumes energy minimisation subject to fracture lengthcontrol. Here, the energy of the system (1) is minimised for a given (fixed) total fracture length increment. This approach is suitable for both stable (5) and unstable (6) crack growth regimes; however, the fracture front is required to be stable (2). In other words, the energy function (1) needs to be convex at least within the constrained solution space. The second strategy assumes load-control. Here, the external load magnitude is incremented which is then followed by the extension of the crack tips that have supercritical crack tip energy release rates. The method is limited to stable fracture growth (5) and a stable fracture front (2). In other words, the energy function (1) needs to be convex. Finally, the third strategy uses the gradient of the total energy function to find the extended crack tip configuration that minimises the energy function subject to the constraint of a fixed total crack increment length. The method is aimed at solving general crack growth problems involving stable (5) or unstable (6) crack growth regimes as well as stable (2) or unstable (3) or partially stable (4) fracture front configuration. In other words, the energy function (1) can generally be non-convex.

\subsection{Crack growth by fractured length control}

The present formulation supposes that the displacement field $\mathbf{u}^{k}$ is automatically obtained by the solution to the discrete equilibrium problem (refer to Part-I Section 6.1) given the variables $\left\{\Delta \boldsymbol{\ell}^{k}, \Delta \boldsymbol{\theta}^{k}\right\}$. Consequently, the evolution of cracks can be considered as the successive minimisation of $\mathcal{E}^{k}=\mathcal{E}\left(\Delta \boldsymbol{\ell}^{k}, \Delta \boldsymbol{\theta}^{k}\right)$ with respect to $\left\{\Delta \boldsymbol{\ell}^{k}, \Delta \boldsymbol{\theta}^{k}\right\}$ for the discrete times $k \in\{1,2, \ldots\}$. At a given time-step $k$, the constraints imposed on the working-set of crack tips $\mathcal{I}_{\text {tip }}^{k} \subseteq \mathcal{I}_{\text {tip }}$ are: (1) a prescribed unit of fracture growth, i.e. $\sum_{i \in \mathcal{I}_{\text {tip }}^{k}} \Delta \ell_{i}^{k}=\Delta a$, and (2) the condition of fracture growth irreversibility, i.e. $\forall i \in \mathcal{I}_{\text {tip }}^{k}$ $\Delta \ell_{i}^{k} \geq 0$. The minimisation problem can be stated as: 


$$
\begin{aligned}
\text { for : } & k \in\{1,2, \ldots\}, i \in \mathcal{I}_{\text {tip }}^{k} \\
\text { minimise : } & \mathcal{E}^{k}=\mathcal{E}\left(\Delta \boldsymbol{\ell}^{k}, \Delta \boldsymbol{\theta}^{k}\right) \\
\text { subject to : } & \sum \Delta \ell_{i}^{k}=\Delta a, \\
& \Delta \ell_{i}^{k} \geq 0
\end{aligned}
$$

where the displacement field $\mathbf{u}^{k}=\mathbf{u}\left(\Delta \boldsymbol{\ell}^{k}, \Delta \boldsymbol{\theta}^{k}\right)$ is implicitly accounted for in the minimisation of $\mathcal{E}\left(\Delta \boldsymbol{\ell}^{k}, \Delta \boldsymbol{\theta}^{k}\right)$ by requiring $\mathbf{u}^{k}$ to satisfy static equilibrium. As such, the Lagrangian of the constrained minimisation problem (16)-(19) can be defined as:

$$
\forall i \in \mathcal{I}_{\text {tip }}^{k} \quad \mathcal{L}\left(\Delta \ell_{i}^{k}, \Delta \theta_{i}^{k}, \lambda_{s}^{k}, \mu_{i}^{k}\right)=\mathcal{E}\left(\Delta \ell_{i}^{k}, \Delta \theta_{i}^{k}\right)+\lambda_{s}^{k}\left(\mathbf{e}_{i} \Delta \ell_{i}^{k}-\Delta a\right)+\mu_{i}^{k}\left(-\Delta \ell_{i}^{k}\right),
$$

where $\lambda_{s}^{k}>0, \mu_{i}^{k} \geq 0$ are the Karush-Kuhn-Tucker (KKT) multipliers. The necessary (but generally insufficient) set of conditions for a stationary point of $\mathcal{L}^{k}$ to correspond to a global minimiser of $\mathcal{E}^{k}$ (subject to satisfying all constraints) is summarised below:

- The stationarity condition:

$$
\begin{gathered}
\forall i \in \mathcal{I}_{\text {tip }}^{k} \quad \frac{\partial \mathcal{L}^{k}}{\partial \Delta \ell_{i}} \equiv \frac{\partial \mathcal{E}^{k}}{\partial \Delta \ell_{i}}+\lambda_{s}^{k} \mathbf{e}_{i}+\mu_{i}^{k}(-1)=0 \\
\frac{\partial \mathcal{L}^{k}}{\partial \Delta \theta_{i}} \equiv \frac{\partial \mathcal{E}^{k}}{\partial \Delta \theta_{i}}=0 \\
\frac{\partial \mathcal{L}}{\partial \lambda_{s}} \equiv \mathbf{e}_{i} \Delta \ell_{i}^{k}-\Delta a=0
\end{gathered}
$$

- Primal feasibility condition:

$$
\forall i \in \mathcal{I}_{\text {tip }}^{k} \quad \Delta \ell_{i}^{k} \geq 0
$$

- Dual feasibility condition:

$$
\forall i \in \mathcal{I}_{\text {tip }}^{k} \quad \mu_{i}^{k} \geq 0
$$

An example solution to the equation system (21)-(25) concerning the problem of two crack tips is shown graphically in Figure 2 .

In practice, it is convenient to disregard the dual feasibility condition (25) and the associated $\mu$-multipliers from the standard Lagrangian form (20) as this adds no practical advantage over the primal feasibility condition $\Delta \ell_{i}^{k} \geq 0$ by virtue of the simplicity of the inequality constraint. In other words, the inequality constraint $\Delta \ell_{i}^{k} \geq 0$ will be 


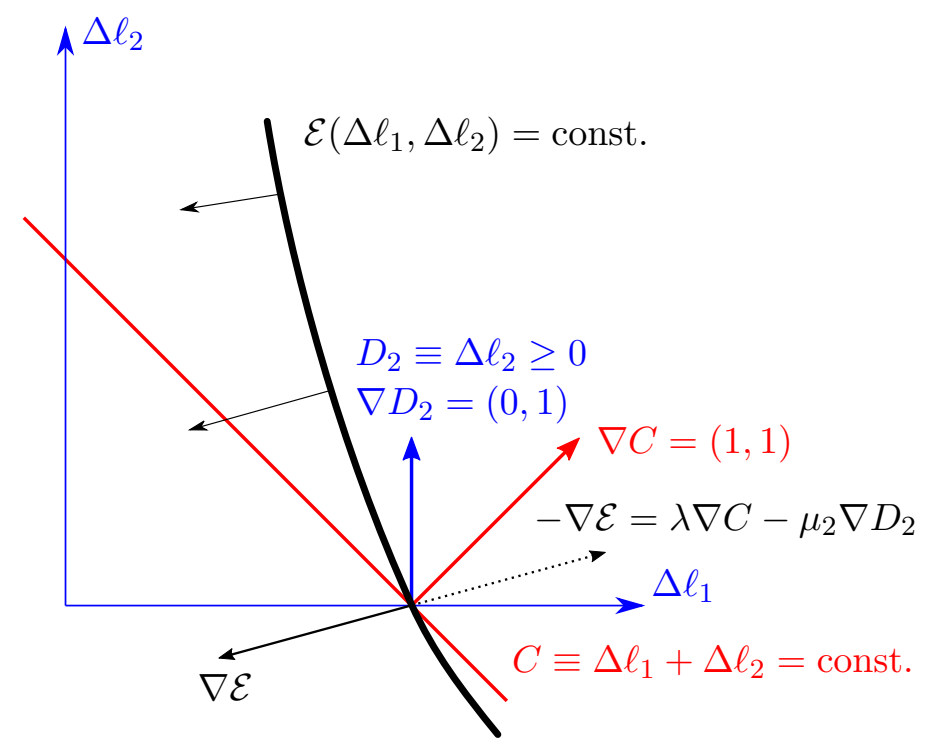

Figure 2: Graphical representation of the critical point of the Lagrangian with one active inequality-constraint. Note that $\lambda>0$ and $\mu_{2}>0$.

enforced directly rather than implicitly via the dual feasibility constraint $\mu_{i}^{k} \geq 0$. The system of non-linear equations (21)-(23) that stems from the stationary principle of the Lagrangian can be linearised and put in an iterative form using Newton's formula:

$$
\left[\begin{array}{ccc}
\frac{\partial \mathcal{E}(\Delta \boldsymbol{\ell}, \Delta \boldsymbol{\theta})}{\partial \Delta \ell_{i} \partial \Delta \ell_{j}} & \frac{\partial \mathcal{E}(\Delta \boldsymbol{\ell}, \Delta \boldsymbol{\theta})}{\partial \Delta \ell_{i} \partial \Delta \theta_{j}} & \mathbf{e}_{i} \\
\frac{\partial \mathcal{E} \Delta \boldsymbol{L}, \Delta \boldsymbol{\theta})}{\partial \Delta \theta_{i} \partial \Delta \ell_{j}} & \frac{\partial \mathcal{E}(\Delta \ell, \Delta \boldsymbol{\theta})}{\partial \Delta \theta_{i} \partial \Delta \theta_{j}} & 0 \\
\mathbf{e}_{j} & 0 & 0
\end{array}\right]_{k, m}\left[\begin{array}{c}
\delta \Delta \ell_{j} \\
\delta \Delta \theta_{j} \\
\delta \lambda_{s}
\end{array}\right]_{k, m}=-\left[\begin{array}{c}
\frac{\partial \mathcal{E}(\Delta \boldsymbol{\ell}, \Delta \boldsymbol{\theta})}{\partial \Delta \ell_{i}}+\mathbf{e}_{i} \lambda_{s} \\
\frac{\partial \mathcal{E}(\Delta \boldsymbol{\ell}, \Delta \boldsymbol{\theta})}{\partial \Delta \theta_{i}} \\
\mathbf{e}_{i} \Delta \ell_{i}-\Delta a
\end{array}\right]_{k, m}
$$

The linearised system of equations can be solved to determine the change in the solution $\left\{\delta \Delta \boldsymbol{\ell}, \delta \Delta \boldsymbol{\theta}, \delta \lambda_{s}\right\}^{k, m}$ at time-step $k \in\{1,2, \ldots\}$ and iteration number $m \in\{1,2, \ldots\}$. However, it is practical to make a few simplifications beforehand. Firstly, the system of equations (26) can be rearranged in order to solve for the multiplier $\lambda_{s}^{k}$ directly. Secondly, it is convenient to suppose that the initial (trial) solution $\Delta \ell^{k, m=1}$ satisfies the equality constraint (23) from the beginning. This way, the residual of the constraint equation vanishes for all iterations $m$ making it easier to maintain the feasibility of the iterated solution with regard to the inequality constraints (24). Finally, the derivatives of the total energy function can be substituted with the corresponding energy release rates, as defined by equations (7)-(15). Altogether, the change in the solution is written as: 


$$
\left[\begin{array}{c}
\delta \Delta \boldsymbol{\ell} \\
\delta \Delta \boldsymbol{\theta} \\
\lambda_{s}
\end{array}\right]_{k, m}=-\left[\begin{array}{ccc}
H_{\mathrm{s}} & H_{\mathrm{m}} & -\mathbf{e} \\
H_{\mathrm{m}}^{\mathrm{T}} & H_{\theta} & 0 \\
-\mathbf{e}^{\mathrm{T}} & 0 & 0
\end{array}\right]_{k, m}^{-1}\left[\begin{array}{c}
G_{\mathrm{s}}-G_{\mathrm{c}} \\
G_{\theta} \\
0
\end{array}\right]_{k, m}
$$

where it can be asserted by inspection of the third equation that the net change in the total crack extension length will be zero, i.e. $\sum_{i \in \mathcal{I}_{\text {tip }}^{k, m}} \delta \Delta \ell_{i}^{k, m}=0$. In other words, the equality constraint (23) that is satisfied by current solution $\Delta \ell^{k, m}$ will likewise be satisfied by the updated solution $\Delta \ell^{k, m+1}=\Delta \boldsymbol{\ell}^{k, m}+\delta \Delta \boldsymbol{\ell}^{k, m}$. Another requirement of $\Delta \ell^{k, m+1}$ is to satisfy all inequality constraints (24), i.e. $\forall i \in \mathcal{I}_{\text {tip }}^{k, m} \Delta \ell_{i}^{k, m+1} \geq 0$. This can be enforced by scaling the solution change $\{\delta \Delta \boldsymbol{\ell}, \delta \Delta \boldsymbol{\theta}\}^{k, m}$ by a factor $w^{m} \geq 0$. The factor is determined by considering the most imminent inequality constraint:

$$
w^{m}=\min \left(\frac{\Delta \ell_{i}^{m}}{-\delta \Delta \ell_{i}^{m}}, 1\right), \text { where } i \in \mathcal{I}_{\text {tip }}^{k, m}
$$

Equation (28) states that the maximum change in the crack tip extension solution that still maintains feasibility of $\Delta \boldsymbol{\ell}^{k, m+1}$ (i.e. $\forall i \in \mathcal{I}_{\text {tip }}^{k, m} \Delta \ell_{i}^{k, m+1} \geq 0$ ) is $w^{m} \delta \Delta \boldsymbol{\ell}^{k, m}$. Thus, the solution after the $m$ 'th iteration can be finally obtained as shown:

$$
\left[\begin{array}{c}
\Delta \boldsymbol{\ell} \\
\Delta \boldsymbol{\theta}
\end{array}\right]^{k, m+1}=\left[\begin{array}{c}
\Delta \boldsymbol{\ell} \\
\Delta \boldsymbol{\theta}
\end{array}\right]^{k, m}+w^{m}\left[\begin{array}{c}
\delta \Delta \boldsymbol{\ell} \\
\delta \Delta \boldsymbol{\theta}
\end{array}\right]^{k, m}
$$

The same scaling factor needs to be applied to the change in crack tip kink angles $\delta \Delta \boldsymbol{\theta}^{k, m}$ since they are linearly related to $\delta \Delta \ell^{k, m}$. Note that if $w^{m}=1$ then none of the inequality constraints (24) limits the optimal change in the solution given by (27).

For the subsequent iteration $m+1$, the same working-set of crack tips $\mathcal{I}_{\text {tip }}^{k, m+1} \leftarrow \mathcal{I}_{\text {tip }}^{k, m}$ can be assumed. If it is found that the change in the solution is prevented by some inequality constraint, say at crack tip $i \in \mathcal{I}_{\text {tip }}^{k, m+1}$ because $w^{m+1}=0$, then this crack tip is discarded from the working-set $\mathcal{I}_{\text {tip }}^{k, m+1}$. The solution at iteration $m+1$ is recomputed considering the updated working-set $\mathcal{I}_{\text {tip }}^{k, m+1} \leftarrow \mathcal{I}_{\text {tip }}^{k, m+1} \backslash i$. The $m$-iterations are continued (updating the working-set as needed) until the solution converges for time-step $k$. Upon convergence, the solution is advanced to the subsequent time-step $k+1$ and the iterative solution process repeated for a new set of candidate crack tips $\mathcal{I}_{\text {tip }}^{k+1, m=1} \subseteq \mathcal{I}_{\text {tip }}^{k}$.

For the present algorithm to converge to the globally minimising solution of the energy function $\mathcal{E}^{k}=\mathcal{E}\left(\Delta \boldsymbol{\ell}^{k}, \Delta \boldsymbol{\theta}^{k}\right)$ using a single trial solution, the function needs to be convex within the admissible solution space, as defined by the constraint equations (18) and (19). The second order sufficiency condition for $\mathcal{E}^{k}$ to be minimised with respect to the set $\mathcal{I}_{\text {tip }}^{k, m \rightarrow \infty}$ of $n_{\text {tip }}^{k, m}=\left|\mathcal{I}_{\text {tip }}^{k, m \rightarrow \infty}\right|$ of crack tip extensions can be generally given as: 


$$
\begin{gathered}
\forall \delta \Delta \boldsymbol{\ell} \in\left\{\boldsymbol{v} \in \mathbb{R}^{n_{\mathrm{tip}}^{k, m}}: \sum_{i=1}^{n_{\mathrm{tip}}^{k, m}} v_{i}=0\right\}, \quad \forall \delta \Delta \boldsymbol{\theta} \in \mathbb{R}^{n_{\mathrm{tip}}^{k, m}} \\
{\left[\begin{array}{ll}
\delta \Delta \ell_{i} & \delta \Delta \theta_{i}
\end{array}\right]\left[\begin{array}{cc}
H_{\mathrm{s} i j} & H_{\mathrm{m} i j} \\
H_{\mathrm{m} j i} & H_{\mathrm{s} i j}
\end{array}\right]_{k, m} \quad\left[\begin{array}{l}
\delta \Delta \ell_{j} \\
\delta \Delta \theta_{j}
\end{array}\right] \leq 0}
\end{gathered}
$$

The admissible variation $\delta \Delta \boldsymbol{\ell}$ can be expressed in terms of an arbitrary variation $\delta \Delta \boldsymbol{\ell}^{*} \in$ $\mathbb{R}^{n_{\text {tip }}^{k, m}}$ by considering the projection of $\delta \Delta \ell^{*}$ onto the admissible $(n-1)$-dimensional plane defined by the zero-variation of the constraint equation $(18)$, i.e. $\sum_{i \in \mathcal{I}_{\text {tip }}^{k, m}} \delta \Delta \ell_{i}=0$ :

$$
\begin{aligned}
\delta \Delta \ell_{i} & =\left(I_{i j}-\frac{\mathbf{e}_{i} \mathbf{e}_{j}}{\mathbf{e}_{i} \mathbf{e}_{i}}\right) \delta \Delta \ell_{j}^{*} \\
\delta \Delta \ell_{i} & =\delta \Delta \ell_{i}^{*}-\operatorname{mean}\left(\delta \Delta \ell^{*}\right)
\end{aligned}
$$

Hence, the second order sufficiency condition (30) can be equivalently written as:

$$
\begin{gathered}
\forall \delta \Delta \boldsymbol{\ell}, \delta \Delta \boldsymbol{\theta} \in \mathbb{R}^{n_{\mathrm{tip}}^{k, m}} \\
{\left[\begin{array}{ll}
\delta \Delta \ell_{i} & \delta \Delta \theta_{i}
\end{array}\right]\left[\begin{array}{cc}
H_{\mathrm{s} i j}^{*} & H_{\mathrm{m} i j}^{*} \\
H_{\mathrm{m} j i}^{*} & H_{\theta i j}
\end{array}\right]_{k, m}\left[\begin{array}{l}
\delta \Delta \ell_{j} \\
\delta \Delta \theta_{j}
\end{array}\right] \leq 0}
\end{gathered}
$$

where $H_{\mathrm{s}}^{*}$ and $H_{\mathrm{m}}^{*}$ are defined as follows:

$$
\begin{aligned}
& H_{\mathrm{s} i j}^{*}=\left(I_{i q}-\frac{\mathbf{e}_{i} \mathbf{e}_{q}}{\mathbf{e}_{i} \mathbf{e}_{i}}\right) H_{\mathrm{s} q r}\left(I_{r j}-\frac{\mathbf{e}_{r} \mathbf{e}_{j}}{\mathbf{e}_{i} \mathbf{e}_{i}}\right) \\
& H_{\mathrm{m} i j}^{*} \equiv\left(H_{\mathrm{m}}^{* \mathrm{~T}}\right)_{j i}=\left(I_{i q}-\frac{\mathbf{e}_{i} \mathbf{e}_{q}}{\mathbf{e}_{i} \mathbf{e}_{i}}\right) H_{\mathrm{m} q j}
\end{aligned}
$$

In summary, the algorithm described in this section is suitable for stable/unstable competing/non-competing crack growth; however, it is required of $\mathcal{E}\left(\Delta \boldsymbol{\ell}^{k}, \Delta \boldsymbol{\theta}^{k}\right)$ to be convex within the solution space defined by $(18)$ and $(19)$. If this condition is met, the solution that minimises $\mathcal{E}\left(\Delta \boldsymbol{\ell}^{k}, \Delta \boldsymbol{\theta}^{k}\right)$ is determined in a single trial. Otherwise, if $\mathcal{E}\left(\Delta \boldsymbol{\ell}^{k}, \Delta \boldsymbol{\theta}^{k}\right)$ is non-convex, the minimisation algorithm is generally not robust (even if multiple trials are attempted) since the solution can converge to a local minimiser.

In solving non-convex optimisation problems it can be more reliable to use a gradientdescent solution algorithm whereby the iterated solution is improved upon by advancing it along the admissible direction that leads to the greatest decrease in the objective function. Before we describe our gradient-descent solution strategy it is worth outlining another approach for the sake of completeness. The following method is based on external load-control and it is exclusively used to resolve stable crack growth (5) under the conditions of a stable fracture front (2), i.e. the energy function must be convex. 


\subsection{Crack growth by external load control}

Crack growth stability depends on a number of factors. Stable crack growth in a solid can be attained for certain geometrical and loading configurations. Some examples include: the double cantilever problem or the wedge-splitting problem $(2 \mathrm{D} / 3 \mathrm{D})$ under displacement control 33, a small embedded crack $(2 \mathrm{D} / 3 \mathrm{D})$ with counter-balancing point loads applied on the top and bottom crack surfaces [31, a pull-out test (3D) whereby a conical shape crack surface develops with an expanding circular fracture front 27 , 3]. Another aspect that tends to promote stable crack growth (at least in the short term) is an increasing $G_{\mathrm{c}}$ by virtue of an expanding plastic region around the crack tip. Finally, environmental conditions can also play a part in crack growth stability in the phenomenon called static fatigue 25,32 . The chemically active environment can locally reduce the material's resistance to fracture growth well below its critical value that can be observed in an inert environment. Thus, as a crack propagates and exposes virgin material, the effective $G_{\mathrm{c}}$ tends increase. A common example of environmentally assisted crack growth is the slow fracture of glass in the presence of moisture $7,8,32$.

Let us suppose a solid geometry and boundary conditions that give rise to a convex total energy function $\mathcal{E}(\Delta \boldsymbol{\ell}, \Delta \boldsymbol{\theta})$ with respect to all possible crack tip extensions $\Delta \boldsymbol{\ell} \in \mathbb{R}^{n_{\mathrm{tip}}^{k}}$ and their angular orientations $\Delta \boldsymbol{\theta} \in \mathbb{R}^{n_{\text {tip }}^{k}}$, where $n_{\text {tip }}^{k}$ is the number of crack tips that may grow at time-step $k$. In this case, the Hessian of $\mathcal{E}(\Delta \boldsymbol{\ell}, \Delta \boldsymbol{\theta})$ is positive definite with respect to the spatial perturbations of the crack tips. Equivalently, it can be stated that the matrix of the rates of the crack tip energy release rates is negative definite:

$$
\begin{gathered}
\forall \delta \Delta \boldsymbol{\ell}, \delta \Delta \boldsymbol{\theta} \in \mathbb{R}^{n_{\mathrm{tip}}^{k}} \\
{\left[\begin{array}{ll}
\delta \Delta \ell_{i} & \delta \Delta \theta_{i}
\end{array}\right]\left[\begin{array}{cc}
H_{\mathrm{s} i j} & H_{\mathrm{m} i j} \\
H_{\mathrm{m} j i} & H_{\mathrm{s} i j}
\end{array}\right]_{k}\left[\begin{array}{l}
\delta \Delta \ell_{j} \\
\delta \Delta \theta_{j}
\end{array}\right] \leq 0}
\end{gathered}
$$

At time $k$ the external load is increased by $\Delta \mathbf{t}^{k}$. Subsequently, the system is generally not in a state of equilibrium. The new equilibrium state will be obtained by minimising $\mathcal{E}\left(\Delta \boldsymbol{\ell}^{k}, \Delta \boldsymbol{\theta}^{k}\right)$ with respect to $\{\Delta \boldsymbol{\ell}, \Delta \boldsymbol{\theta}\}^{k}$ subject to the irreversibility constraint: $\forall i \in \mathcal{I}_{\text {tip }}^{k}$ $\Delta \ell_{i}^{k} \geq 0$. Since the energy function is convex, it suffices to consider a single trial solution. For example, the maximum hoop stress criterion can be used to uniformly extend all crack tips in the working-set $\mathcal{I}_{\text {tip }}^{k, m=1} \subseteq \mathcal{I}_{\text {tip }}^{k}$ as an initial solution. The linearised system of equations to be solved at an iteration step $m \in\{1,2, \ldots\}$ is similar to that of the previous formulation that was based on fracture-length control. The difference is that the equality-constraint 19 is left out. The resulting set of equations simply reads:

$$
\left[\begin{array}{ll}
\frac{\partial \mathcal{E}(\Delta \boldsymbol{\ell}, \Delta \boldsymbol{\theta})}{\partial \Delta \ell_{i} \partial \Delta \ell_{j}} & \frac{\partial \mathcal{E}(\Delta \boldsymbol{\ell}, \Delta \boldsymbol{\theta})}{\partial \Delta \ell_{i} \partial \Delta \theta_{j}} \\
\frac{\partial \mathcal{E}(\Delta \boldsymbol{\ell}, \Delta \boldsymbol{\theta})}{\partial \Delta \theta_{i} \partial \Delta \ell_{j}} & \frac{\partial \mathcal{E}(\Delta \boldsymbol{\ell}, \Delta \boldsymbol{\theta})}{\partial \Delta \theta_{i} \partial \Delta \theta_{j}}
\end{array}\right]_{k, m}\left[\begin{array}{l}
\delta \Delta \ell_{j} \\
\delta \Delta \theta_{j}
\end{array}\right]_{k, m}=-\left[\begin{array}{l}
\frac{\partial \mathcal{E}(\Delta \boldsymbol{\ell}, \Delta \boldsymbol{\theta})}{\partial \Delta \ell_{i}} \\
\frac{\partial \mathcal{E}\left(\Delta, \boldsymbol{\ell}_{i}, \Delta \boldsymbol{\theta}\right)}{\partial \Delta \theta_{i}}
\end{array}\right]_{k, m}
$$


The solution change $\{\delta \Delta \boldsymbol{\ell}, \delta \Delta \boldsymbol{\theta}\}^{k, m}$ can be obtained straightforwardly from (37). For convenience, we substitute the definitions of the energy release rates (7)-(15) in place of the derivatives of the energy function appearing in (37). This subsequently yields:

$$
\left[\begin{array}{l}
\delta \Delta \boldsymbol{\ell} \\
\delta \Delta \boldsymbol{\theta}
\end{array}\right]_{k, m}=-\left[\begin{array}{cc}
H_{s} & H_{m} \\
H_{m}^{\mathrm{T}} & H_{\theta}
\end{array}\right]_{k, m}^{-1}\left[\begin{array}{c}
G_{\mathrm{s}}-G_{c} \\
G_{\theta}
\end{array}\right]_{k, m}
$$

Since the total energy function is assumed to be convex, the tangent matrix in (38) will be negative definite. Consequently, the problem of determining the new equilibrium crack tip positions for a given external load increment $\Delta \mathbf{t}^{k}$ effectively involves growing cracks to the point that no crack tip exceeds the critical value of the energy release rate $G_{\mathrm{c}}$. The solution process for time-step $k$ is analogous to that of Section 3.1. In short, the solution $\{\Delta \boldsymbol{\ell}, \Delta \boldsymbol{\theta}\}^{k}$ is determined iteratively by solving $(38)$ for $\{\delta \Delta \boldsymbol{\ell}, \delta \Delta \boldsymbol{\theta}\}^{k, m}$ and updating crack tip positions according to equations $(28)$ and $(29)$ in order to uphold the constraint of fracture irreversibility, i.e. $\forall i \in \mathcal{I}_{\text {tip }}^{k, m} \Delta \ell_{i}^{k, m+1} \geq 0$. The solution converges for time-step $k$ when there are no super-critical crack tips, i.e. $\forall i \in \mathcal{I}_{\text {tip }}^{k} G_{\mathrm{s} i}^{k} \leq G_{\mathrm{C}}$, and the rotational energy release rates vanish, i.e. $\forall i \in \mathcal{I}_{\text {tip }}^{k} G_{\theta i}^{k}=0$. Thereafter, the solution time-step is advanced to $k+1$, the external load is incremented by $\Delta \mathbf{t}^{k+1}$ and the iterative solution process is repeated for a new set of crack tips $\mathcal{I}_{\text {tip }}^{k+1, m=1} \subseteq \mathcal{I}_{\text {tip }}^{k}$.

After each time-step, it is important to increase the load magnitude by a small amount in order to achieve small crack tip increments so that the evolution of the fracture surface can be captured precisely. The interested reader can refer to the works $[9,10]$ for details on a 3D implementation regarding planar crack growth. The main shortcoming of the load-control scheme is that its application is limited to a stable fracture front configuration (2) and stable crack growth (5), i.e. the energy function needs to be convex. In practice, however, a convex energy function rarely arises in fracture problems, unless the material's resistance to fracture increases at a sufficiently rapid rate.

\subsection{Energy-gradient based crack growth}

Neither one of the two preceding solution strategies is suitable for solving the general case of crack growth since these methods will generally fail if the energy function is nonconvex. To overcome this limitation, an alternative solution method is pursued whereby a mixture of an extremum-based and a gradient-descent energy minimisation techniques (i.e. a staggered solution approach) is used. The two techniques are employed alternately to determine the directions and the lengths of the crack tip extensions in turn. The two sets of variables, namely: the crack tip extension angles $\Delta \boldsymbol{\theta}=\left\{\Delta \theta_{i}\right\}_{i \in \mathcal{I}_{\text {tip }}}$ and the crack tip extension lengths $\Delta \boldsymbol{\ell}=\left\{\Delta \ell_{i}\right\}_{i \in \mathcal{I}_{\text {tip }}}$ are considered as decoupled sets of variables at each iteration, i.e. the inter-dependence (11) is ignored. However, with each iteration, the crack tip extension angles are updated to account for the changes in the extension 
lengths. This is possible because the interactions between $\Delta \boldsymbol{\ell}$ and $\Delta \boldsymbol{\theta}$ will vanish in the limit the crack tip extensions are very small (regardless of their relative lengths).

At a typical time-step $k \in\{1,2, \ldots\}$, the first part of an iteration $m \in\{1,2, \ldots\}$ involves determining the crack growth directions for the working-set of crack tips $\mathcal{I}_{\text {tip }}^{k, m} \subseteq \mathcal{I}_{\text {tip }}$. A convex minimisation approach is applied to minimise $\mathcal{E}^{k, m}=\mathcal{E}\left(\Delta \boldsymbol{\theta}^{k, m} ; \Delta \boldsymbol{\ell}^{k, m}\right)$ with respect to $\Delta \boldsymbol{\theta}^{k, m}$ for the crack tip extension lengths $\Delta \boldsymbol{\ell}^{k, m}$. Specifically, the problem is to find the crack extension directions that yield zero rotational energy release rates:

$$
\begin{aligned}
& \forall i \in \mathcal{I}_{\text {tip }}^{k, m} \text { find } \Delta \theta_{i}^{k, m} \in \mathbb{R}_{(-\pi, \pi)} \text { such that } \forall j \in \mathcal{I}_{\text {tip }}^{k, m} G_{\theta j}^{k, m}=0 \\
& \text { assuming } \forall \delta \Delta \boldsymbol{\theta} \in \mathbb{R}^{n_{\text {tip }}^{k, m}} H_{\theta_{i j}}^{k, m} \delta \Delta \theta_{i} \delta \Delta \theta_{j} \leq 0 \text { where } i, j \in \mathcal{I}_{\text {tip }}^{k, m}
\end{aligned}
$$

The solution to the non-linear problem $(39)$ in terms of the crack tip extension angles $\Delta \boldsymbol{\theta}^{k, m}$ is determined using Newton's method. Thus, the $n^{\prime}$ th iteration step reads:

$$
\Delta \theta_{i}^{k, m, n+1}=\Delta \theta_{i}^{k, m, n}-\left(H_{\theta}^{-1}\right)_{i j}^{k, m, n} G_{\theta_{j}}^{k, m, n} \quad \text { where } i, j \in \mathcal{I}_{\text {tip }}^{k, m}
$$

The $n$-iterations are repeated until the change in two consecutive solutions is sufficiently small, e.g. $\left\|\Delta \theta^{k, m, n+1}-\Delta \theta^{k, m, n}\right\|_{\infty}<\epsilon$ where $\epsilon$ is a small positive number. In the second part of the $m$ 'th iteration, the crack tip extension lengths are updated by applying a gradient-descent scheme to minimise the energy of the system with respect to crack tip extension lengths $\Delta \boldsymbol{\ell}^{k, m}$ while keeping constant $\Delta \boldsymbol{\theta}^{k, m} \leftarrow \Delta \boldsymbol{\theta}^{k, m, n \rightarrow \infty}$, as determined in (41). In this case, the change in the crack tip extension lengths should theoretically take place in the direction of the vector $d_{i}^{k, m}=G_{\mathrm{s}_{i}}{ }^{k, m}-\operatorname{mean}\left(G_{\mathrm{s}}{ }^{k, m}\right)$ where $i \in \mathcal{I}_{\text {tip }}^{k, m}$ such that the constraint of a unit total fracture increment is preserved, i.e. $\sum_{i \in \mathcal{I}_{\text {tip }}^{k, m}} d_{i}^{k, m}=0$.

However, the present numerical discretisation (XFEM) assumes fixed-length crack tip extensions, i.e. crack tip growth can only be switched either on or off. For this reason, the gradient-descent approach needs to be adapted. Our solution strategy is to update the crack tip extension lengths according to the following boolian-like criterion 1

$$
\forall i \in \mathcal{I}_{\text {tip }}^{k, m} \quad \Delta \ell_{i}^{k, m+1}= \begin{cases}\Delta \ell_{\text {inc }} & \text { if } G_{\mathrm{s} i}^{k, m} \geq \operatorname{mean}\left(G_{\mathrm{s}}{ }^{k, m}\right) \\ 0 & \text { otherwise. }\end{cases}
$$

This step effectively results in the annihilation of the crack tip extensions that are energetically favoured to undergo some amount of closure and the retention of the crack tip extensions that are energetically favoured to undergo further opening. The new

\footnotetext{
${ }^{1} \mathrm{~A}$ possible alternative criterion when crack tip interactions can be considered insignificant (e.g. the inequality 43 is false) is: $G_{\mathrm{s}_{i}}{ }^{k, m} \geq \beta \max \left(G_{\mathrm{s}}{ }^{k, m}\right)$, where $0<\beta<1$ is a slack factor, e.g. $\beta=0.98$.
} 


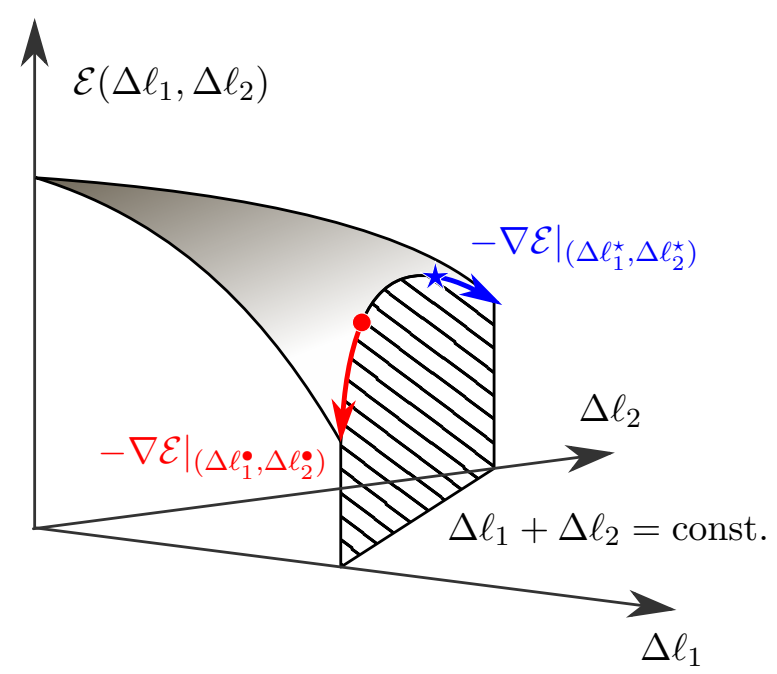

Figure 3: Converged solution dependence on the starting point. The idealised fracture problem is governed by a concave energy function within the admissible solution space. Using a gradient-descent method can lead to a sub-optimal solution, depending on the starting point. Hence, the gradient-descent approach will generally not be robust.

working-set of crack tips $\mathcal{I}_{\text {tip }}^{k, m+1} \subseteq \mathcal{I}_{\text {tip }}^{k, m}$ for the next iteration $m+1$ consists only of the remaining crack tip extensions, i.e. $\mathcal{I}_{\text {tip }}^{k, m+1}=\left\{i \in \mathcal{I}_{\text {tip }}^{k, m}: \Delta \ell_{i}^{k, m+1}=\Delta \ell_{\text {inc }}\right\}$.

Although the proposed gradient-descent approximation in 42 is rough, the method will converge to the energetically optimal fracture paths provided that the crack tip extensions are small and at least one of the following two conditions holds true:

1. crack tip competition is absent (43), or

2. the total energy function is convex (36)

In other words, under condition (1.) the energy function can be convex or non-convex (e.g. concave or a generalised saddle function), and under condition (2.) crack tip competition can be resolved naturally by the proposed gradient-descent approach (42). For any case that satisfies (1.) or (2.) a single trial solution is sufficient to converge to the optimal solution for a given discretisation. For example, uniformly incrementing all promising crack tips in the set $\mathcal{I}_{\text {tip }}^{k, m=1} \subseteq \mathcal{I}_{\text {tip }}^{k}$ is an adequate initial guess. On the other hand, the current method can not be applied robustly to solve the case of competing crack growth at the instance of a non-convex energy function. This is due to the possibility of multiple locally optimal solutions that the algorithm can converge to, depending on the starting solution (see for example Figure 3).

The advantage of the proposed gradient-descent approach is that the method is more versatile than the extremum-based convex minimisation methods that were described in 
Sections 3.1 and 3.2 since it can be applied even if the total energy function is non-convex (provided of course that crack competition does not arise). The main shortcoming is the reduced accuracy due to the assumption of fixed-length crack tip extensions - a constraint imposed entirely by the present numerical discretisation - and the reduced convergence due to the staggered iterative solution method (i.e. the crack tip extension lengths $\Delta \boldsymbol{\ell}^{k, m}$ and the extension directions $\Delta \boldsymbol{\theta}^{k, m}$ are decoupled during the $m$-iterations).

The satisfactory performance of our proposed solution approach is verified by a number of fabricated test cases that have been designed to test the method against competing/noncompeting crack growth for convex/non-convex behaviours of the energy function. The numerical results are presented in Section 4. The following section proposes a solution method for resolving competing crack growth in the case of a non-convex energy function while subject to the discretisation constraint of fixed-length crack tip extensions.

\section{Resolving competing crack growth}

Three solution strategies have been described for solving multi-crack growth problems within a discrete framework. The different methods lend themselves to certain types of fracture growth problems better than to others (depending on the shape of the energy function). However, neither of the preceding strategies is completely robust in the case of competing crack growth and an unstable (3) or a partially stable (4) fracture configuration, i.e. when the energy function is non-convex. In solving non-convex minimisation problems, the method of choice is usually some form of a multi-trial gradient-descent approach. However, within the current discretisation that assumes fixed-length crack tip extensions, a multi-trial approach is not directly viable because the essential constraint of a unit total increase in the fracture lengths can not be satisfied by all trial solutions. Because of this difficulty, our approach is to locally approximate the energy function by a quadratic function and use a multi-trial gradient-descent method to minimise it. This enables finding an approximate solution to the fracture extensions and identifying the critical crack tips that should grow. The proposed strategy proves effective in resolving competing crack growth mainly because it can identify the critical crack tips accurately. It turns out that extending only the critical crack tips is more important to the overall solution accuracy than the accuracy of the crack tip extension lengths themselves. The following two sub-sections focus on the criterion for detecting crack tip competition and on the solution method for determining the competing crack tip extension lengths.

\subsection{Detection of crack tip competition}

A multi-trial gradient-descent solution approach is necessary when two conditions are met: (1) the crack tips in the set $\mathcal{I}_{\text {tip }}^{k}$ are competing and (2) the energy function $\mathcal{E}\left(\Delta \boldsymbol{\ell}^{k} ; \Delta \boldsymbol{\theta}^{k}\right)$ is non-convex with respect to $\Delta \boldsymbol{\ell}^{k}$ (as determined following the solution to (41) for $\Delta \boldsymbol{\theta}^{k}$ ). Within a discrete framework crack tips can effectively be considered as 
competing when their energy release rates $\left\{G_{\mathrm{s} i}\right\}_{i \in \mathcal{I}_{\mathrm{tip}}^{k}}$ are sufficiently close such that the effects of the higher order terms, namely: $H_{\mathrm{s}}, H_{\mathrm{m}}$ and $H_{\theta}$, acting over the finite-length crack tip extensions become important to the solution outcome. A simple check for assessing crack tip competition (i.e. the significance their interactions) is:

$$
\underset{i \in \mathcal{I}_{\mathrm{tip}}^{k}}{\arg \max }\left(G_{\mathrm{s} i}^{k}\right) \neq \underset{i \in \mathcal{I}_{\mathrm{tip}}^{k}}{\arg \max }\left(G_{\mathrm{s} i}^{k}-\Delta \ell_{\mathrm{inc}} \sum_{j \in \mathcal{I}_{\mathrm{tip}}^{k}}\left|H_{\mathrm{s} i j}^{k}\right|\right)
$$

This inequality indicates whether the crack tip with the maximum energy release rate (i.e. the critical crack tip) is likely to change during the extension of any of the crack tips in the set $\mathcal{I}_{\text {tip }}^{k}$. In other words, the crack tip interactions are important to the outcome of the solution if the inequality (43) is true. The next step is to assess if $\mathcal{E}\left(\Delta \boldsymbol{\ell}^{k}, \Delta \boldsymbol{\theta}^{k}\right)$ is non-convex. To this end, the second order KKT sufficiency condition (33) can be used. However the condition simplifies if the crack tip extension lengths can be considered to be small. Specifically, the second order cross-interactions due to $H_{\mathrm{m} i j}$ where $i \neq j$ can be ignored since they are remote, whereas the self-interaction $H_{\mathrm{m} i i} \equiv \frac{\partial}{\partial \theta_{i}}\left(-\frac{\partial \mathcal{E}}{\partial \ell_{i}}\right) \equiv \frac{\partial G_{\mathrm{s} i}}{\partial \theta_{i}}$ for $i \in \mathcal{I}_{\text {tip }}^{k}$ can be ignored for the following reason. A viable fracture extension solution must minimise $\mathcal{E}\left(\Delta \boldsymbol{\ell}^{k}, \Delta \boldsymbol{\theta}^{k}\right)$ with respect to $\Delta \boldsymbol{\theta}^{k}$ meaning that the crack tip energy release rates $G_{\mathrm{s} i}$ at the individual crack tips $i \in \mathcal{I}_{\text {tip }}^{k}$ must be maximised (or stationary), i.e. $\forall i \in \mathcal{I}_{\text {tip }}^{k} \lim _{\Delta \ell_{i} \rightarrow 0^{+}} \partial G_{\mathrm{s} i} /\left.\partial \theta_{i}\right|_{\Delta \ell}=0$. Therefore, if the crack tip extensions are small, the following criterion is sufficient to assess if the energy function is non-convex:

$$
\exists \delta \Delta \ell \in \mathbb{R}^{n_{\mathrm{tip}}^{k}} \quad H_{\mathrm{s} i j}^{* k} \delta \Delta \ell_{i} \delta \Delta \ell_{j}>0
$$

The matrix $H_{\mathrm{s}}^{*}$ (defined by (34)) is the projection of $H_{\mathrm{s}}$ onto the constrained solution space that imposes a fixed unit of fracture length advance as specified by (18). For example, the particular case of competing crack growth that is depicted in Figure 3 satisfies 44); hence, a multi-trial gradient-descent solution approach is generally required.

\subsection{Determination of critical crack tips}

Extending the wrong crack tips in the case of competing crack growth and a non-convex energy function can result in the complete departure from the critical fracture path. If, on the other hand, the critical set of tips can be identified exactly, the precision of the crack tip extension lengths has a lesser effect on the global accuracy of the fracture path. In other words, so long as only the critical crack tips are advanced, the critical fracture path will not be lost. Therefore, the main challenge with fixed-length crack tip extensions within a discretisation is the identification of the critical crack tips.

To determine the set of critical crack tips that should growth at the instance of competing crack growth 43 it is proposed to construct an explicit approximation to the energy 
function and find the crack tip extension solution that minimises this energy function. Although the solution to the crack tip extension lengths - herein referred to as the offline solution - will be approximate, it should suffice to identify the critical crack tips exactly. Once the critical tips are determined, the transition from the offline solution $\left(\Delta \tilde{\ell}^{k}\right)$ to the discrete solution $\left(\Delta \ell^{k}\right)$ can be made via a coarsening operation which essentially involves rounding-off the offline solution to fit the discretisation (e.g. XFEM).

Assuming small crack tip extensions and small changes in the fracture front configuration relative to a reference solution point $\left\{\Delta \boldsymbol{\ell}_{0}^{k}, \Delta \boldsymbol{\theta}_{0}^{k}\right\}$ the energy function $\mathcal{E}\left(\Delta \boldsymbol{\ell}^{k}, \Delta \boldsymbol{\theta}^{k}\right)$ can be sufficiently well approximated by a quadratic function $\tilde{\mathcal{E}}\left(\Delta \boldsymbol{\ell}^{k}, \delta \Delta \boldsymbol{\theta}^{k}\right)$ using Tailor's series expansion, where $\Delta \boldsymbol{\ell}^{k}=\Delta \boldsymbol{\ell}_{0}^{k}+\delta \Delta \boldsymbol{\ell}^{k}$ and $\Delta \boldsymbol{\theta}^{k}=\Delta \boldsymbol{\theta}_{0}^{k}+\delta \Delta \boldsymbol{\theta}^{k}$ :

$$
\begin{gathered}
\tilde{\mathcal{E}}\left(\Delta \boldsymbol{\ell}^{k}, \delta \Delta \boldsymbol{\theta}^{k}\right)=\mathcal{E}^{k-1}-\left[\left(G_{\mathrm{s} i}^{0}-G_{\mathrm{c}}-H_{\mathrm{s} i j}^{0} \Delta \ell_{0 j}^{k}\right) \Delta \ell_{i}^{k}+G_{\theta i}^{0} \delta \Delta \theta_{i}^{k}+\ldots\right. \\
\left.+\frac{1}{2} H_{\mathrm{s} i j}^{0} \Delta \ell_{i}^{k} \Delta \ell_{j}^{k}+H_{\mathrm{m} i j}^{0} \Delta \ell_{i}^{k} \delta \Delta \theta_{j}^{k}+\frac{1}{2} H_{\theta i j}^{0} \delta \Delta \theta_{i}^{k} \delta \Delta \theta_{j}^{k}\right]
\end{gathered}
$$

Suppose the reference fracture configuration $\left\{\Delta \boldsymbol{\ell}_{0}^{k}, \Delta \boldsymbol{\theta}_{0}^{k}\right\}$ is the trial crack tip extension solution at the instance crack tip competition is detected (43). Furthermore, suppose that all terms in (45) that are superscripted with a nought are computed in the reference configuration, i.e. $(\cdot)^{0}=(\cdot)\left(\Delta \boldsymbol{\ell}_{0}^{k}, \Delta \boldsymbol{\theta}_{0}^{k}\right)$. Since $\mathcal{E}\left(\Delta \boldsymbol{\ell}_{0}^{k}, \Delta \boldsymbol{\theta}_{0}^{k}\right)$ is minimised (stationary) at $\Delta \boldsymbol{\theta}_{0}^{k}$ following the solution to (41), the rotational energy release rates must vanish in (45), i.e. $\forall i \in \mathcal{I}_{\text {tip }}^{k} G_{\theta i}^{0}=0$. Subsequently, the changes in the crack tip kink angles $\delta \Delta \boldsymbol{\theta}^{k}$ can be expressed in terms of the crack tip extension lengths $\Delta \boldsymbol{\ell}^{k}$ by supposing that $\tilde{\mathcal{E}}\left(\Delta \boldsymbol{\ell}^{k}, \Delta \boldsymbol{\theta}^{k}-\Delta \boldsymbol{\theta}_{0}^{k}\right)$ remains stationary with respect to $\Delta \boldsymbol{\theta}^{k}$ for any change in $\Delta \boldsymbol{\ell}^{k}$ :

$$
\begin{gathered}
\left.\delta \Delta \theta_{i}^{k} \frac{\partial}{\partial \theta_{i}}\left(\frac{\partial \tilde{\mathcal{E}}}{\partial \theta_{j}}\right)\right|_{\Delta \ell_{0}^{k}, \Delta \boldsymbol{\theta}_{0}^{k}}+\left.\delta \Delta \ell_{i}^{k} \frac{\partial}{\partial \ell_{i}}\left(\frac{\partial \tilde{\mathcal{E}}}{\partial \theta_{j}}\right)\right|_{\Delta \ell_{0}^{k}, \Delta \boldsymbol{\theta}_{0}^{k}}=0 \\
\delta \Delta \theta_{i}^{k}=-\left(\Delta \ell_{j}^{k}-\Delta \ell_{0 j}^{k}\right) H_{\mathrm{m} j I}^{0}\left(H_{\theta}^{0-1}\right)_{I i}, \quad \text { where } i, j, I \in \mathcal{I}_{\text {tip }}^{k, m}
\end{gathered}
$$

At this point it can be deduced that the variation $\delta \Delta \boldsymbol{\theta}^{k}$ is negligible due to the following three reasons. (1) The long-distance interactions between different crack tips are negligible if the crack tip extensions are small relative to the problem geometry, i.e. $H_{\mathrm{m} i j}^{0} \equiv \frac{\partial G_{\mathrm{s} i}^{0}}{\partial \theta_{j}} \rightarrow 0$ and $H_{\theta i j}^{0} \equiv \frac{\partial G_{\theta_{i}}^{0}}{\partial \theta_{j}} \rightarrow 0$ for $i \neq j$. (2) The self-interaction $H_{\mathrm{m} i i}^{0}$ will tend to vanish for small crack tip extensions since the state of minimum energy with respect to the crack tip kink angles implies that the energy release rates at the individual crack tips are maximum, i.e. $G_{\mathrm{s} i}^{0}$ is stationary such that $H_{\mathrm{m} i i}^{0} \equiv \frac{\partial G_{\mathrm{s} i}^{0}}{\partial \theta_{i}} \rightarrow 0$. Finally, (3) the effect of the self-interaction $\left(H_{\theta}^{0-1}\right)_{i i}$ which is of order $\mathcal{O}\left(1 / \Delta \ell_{0}^{k}\right)$ will cancel out with $\left(\Delta \ell_{j}^{k}-\Delta \ell_{0 j}^{k}\right)$ which is of order $\mathcal{O}\left(\Delta \ell_{0}^{k}\right)$ such that the product of the two terms is of order $\mathcal{O}(1)$, which has no significant influence in (47) since $H_{\mathrm{m}}^{0}$ has been shown to vanish. 
Based on the same reasoning, the last two terms in the expression for $\tilde{\mathcal{E}}\left(\Delta \boldsymbol{\ell}^{k}, \delta \Delta \boldsymbol{\theta}^{k}\right)$ in equation (45) can be disregarded since their influence will be small relative to that of the remaining terms. Consequently, $\tilde{\mathcal{E}}\left(\Delta \boldsymbol{\ell}^{k}, \delta \Delta \boldsymbol{\theta}^{k}\right)$ is predominately governed by $\Delta \boldsymbol{\ell}^{k}$ :

$$
\tilde{\mathcal{E}}\left(\Delta \ell^{k}\right)=\mathcal{E}^{k-1}-\left[\left(G_{\mathrm{s} i}^{0}-G_{\mathrm{c}}-H_{\mathrm{s} i j}^{0} \Delta \ell_{0 j}^{k}\right) \Delta \ell_{i}^{k}+\frac{1}{2} H_{\mathrm{s} i j}^{0} \Delta \ell_{i}^{k} \Delta \ell_{j}^{k}\right]
$$

The approximation above contains sufficient information about the local behaviour of $\mathcal{E}\left(\Delta \boldsymbol{\ell}^{k}, \Delta \boldsymbol{\theta}^{k}\right)$. Therefore, by minimising $\tilde{\mathcal{E}}\left(\Delta \boldsymbol{\ell}^{k}\right)$ it should be possible to obtain the critical crack tips exactly. We will denote by $\Delta \tilde{\ell}^{k} \in \mathbb{R}^{n_{\text {tip }}^{k}}$ the crack extension lengths that are determined as the solution to the following offline energy minimisation problem:

$$
\begin{aligned}
\text { minimise : } & \tilde{\mathcal{E}}^{k}=\tilde{\mathcal{E}}\left(\Delta \tilde{\ell}^{k}\right) \\
\text { subject to : } & \sum_{i \in \mathcal{I}_{\text {tip }}^{k}} \Delta \tilde{\ell}_{i}^{k}=\Delta a \\
& \forall i \in \mathcal{I}_{\text {tip }}^{k} \quad \Delta \tilde{\ell}_{i}^{k} \geq 0 \\
\text { assuming : } & \forall i \in \mathcal{I}_{\text {tip }}^{k} \quad \Delta \ell_{0 i}^{k}=\Delta \ell_{\mathrm{inc}} \\
& \forall i \in \mathcal{I}_{\text {tip }}^{k} \quad \Delta \theta_{0 i}^{k}=\arg \left(G_{\theta i}^{0}=0\right)
\end{aligned}
$$

Although the term in the parenthesis of equation (48) will almost never have all equal elements, which is to say that competing crack growth at any discrete time instance will almost never arise, the purpose of 49 is to resolve crack tip competition as it may arise between two discrete times, i.e. $k$ and $k+1$. With regard to the constant $\Delta a$ in (50), ideally it should be chosen such that the offline solution $\Delta \tilde{\ell}^{k}$ can be reproduced by the discrete solution $\Delta \boldsymbol{\ell}^{k}$. However, this is not generally possible if the offline solution consists of multiple crack tips advancing at different rates (as opposed to the extension of single crack tip). Nonetheless, the value for $\Delta a$ can be chosen to equal the length of a discrete crack tip extension, e.g. $\Delta a=\Delta \ell_{\text {inc }}$. Now, if the offline solution indicates multiple crack tip growth then this leads to infer that the energy function is convex in the vicinity of the critical solution; thus, reproducing the crack tip extensions exactly is not as crucial as growing the right crack tips. For this reason, the coarsening of the offline crack tip extension lengths to fit the discrete framework can work in practice.

The offline energy minimisation problem (49)-(53) can be solved using standard iterative methods such as those used for solving constrained quadratic programming problems. For example, the active-set method that was described in Part-I Section 7.2.3 of this three-part paper can be applied. Refer to Appendix C of Part-I for the solution algorithm or follow the links provided in Section 8 for the Matlab implementation.

As the offline solution is of an arbitrary resolution that generally can not be reproduced by the numerical discretisation, the offline solution will need to be coarsened so as to 
fit it into the discrete framework. One way to do this is to (1) scale the offline crack tip extensions such that the maximum extension equals the discrete increment length and (2) round-off the remaining crack tip extensions relative to the discrete increment length. For example, the offline solution $\Delta \tilde{\ell}^{k}$ can be coarsened to obtain $\Delta \boldsymbol{\ell}^{k}$ as shown:

$$
\Delta \ell_{i}^{k}=\operatorname{round}\left(\frac{\Delta \tilde{\ell}_{i}^{k}}{\max \left(\Delta \tilde{\ell}^{k}\right)}\right) \Delta \ell_{\mathrm{inc}}, \quad \text { where } i \in \mathcal{I}_{\text {tip }}^{k}
$$

Once the discrete crack tip extension lengths $\Delta \boldsymbol{\ell}^{k}$ have been obtained, it is desirable to make a final update to the crack tip extension directions since the reference solution $\Delta \boldsymbol{\theta}_{0}^{k}$ is sub-optimal for the current solution $\Delta \boldsymbol{\ell}^{k}$ (unless $\Delta \boldsymbol{\ell}^{k}=\Delta \boldsymbol{\ell}_{0}^{k}$ ). As the changes in the crack tip kink angles are expected to be small (by virtue of small crack tip extension lengths and, thus, diminishingly small interactions between the extension lengths and the angles), a few iterations of equation (41) should suffice to obtain the optimal solution for $\Delta \boldsymbol{\theta}^{k}$. Any further change in the crack tip extension lengths $\Delta \boldsymbol{\ell}^{k}$ can be ignored.

In summary, at the instance competing crack growth is detected (43) and the energy function happens to be non-convex (44) the single-trial gradient-descent solution approach (refer to Section 3.3) needs to be switched to a multi-trial approach to solve an approximated energy minimisation problem (49)-(53). The so-called offline solution to the crack tip extension lengths is obtained. Finally, this solution is coarsened (54) so that it can be fitted within the discrete framework of fixed-length crack tip extensions.

\subsection{Summary of solution algorithm}

The flowchart of the discrete solution algorithm is given by Figure 4 below. Note that the critical load $\mathbf{t}^{k}$ needs to be determined at the end of every time-step $k$. This can be done by scaling the applied nominal load $\mathbf{t}_{0}^{k}$ by a factor $\lambda_{t}^{k}$. The critical load factor can be determined as $\lambda_{t}^{k}=\sqrt{G_{c} / \max G_{\mathrm{s}}{ }^{k}}$ since the crack tip energy release rates $\left\{G_{\mathrm{s} i}\right\}_{i \in \mathcal{I}_{\text {tip }}^{k}}$ in a linear-elastic solid are proportional to the square of the applied load. Finding the critical load ensures the fracture state is physically admissible, i.e. $\forall i \in \mathcal{I}_{\text {tip }}^{k} G_{\mathrm{s} i} \leq G_{\mathrm{c}}$, and that the evolution of the fractures satisfies the assumption of quasi-statics.

The subsequent section verifies the proposed formulation for solving competing crack growth problems within the discrete framework. Several representative case studies are considered that are designed to test the method's robustness in competing/noncompeting crack growth for convex/non-convex behaviours of the energy function. 


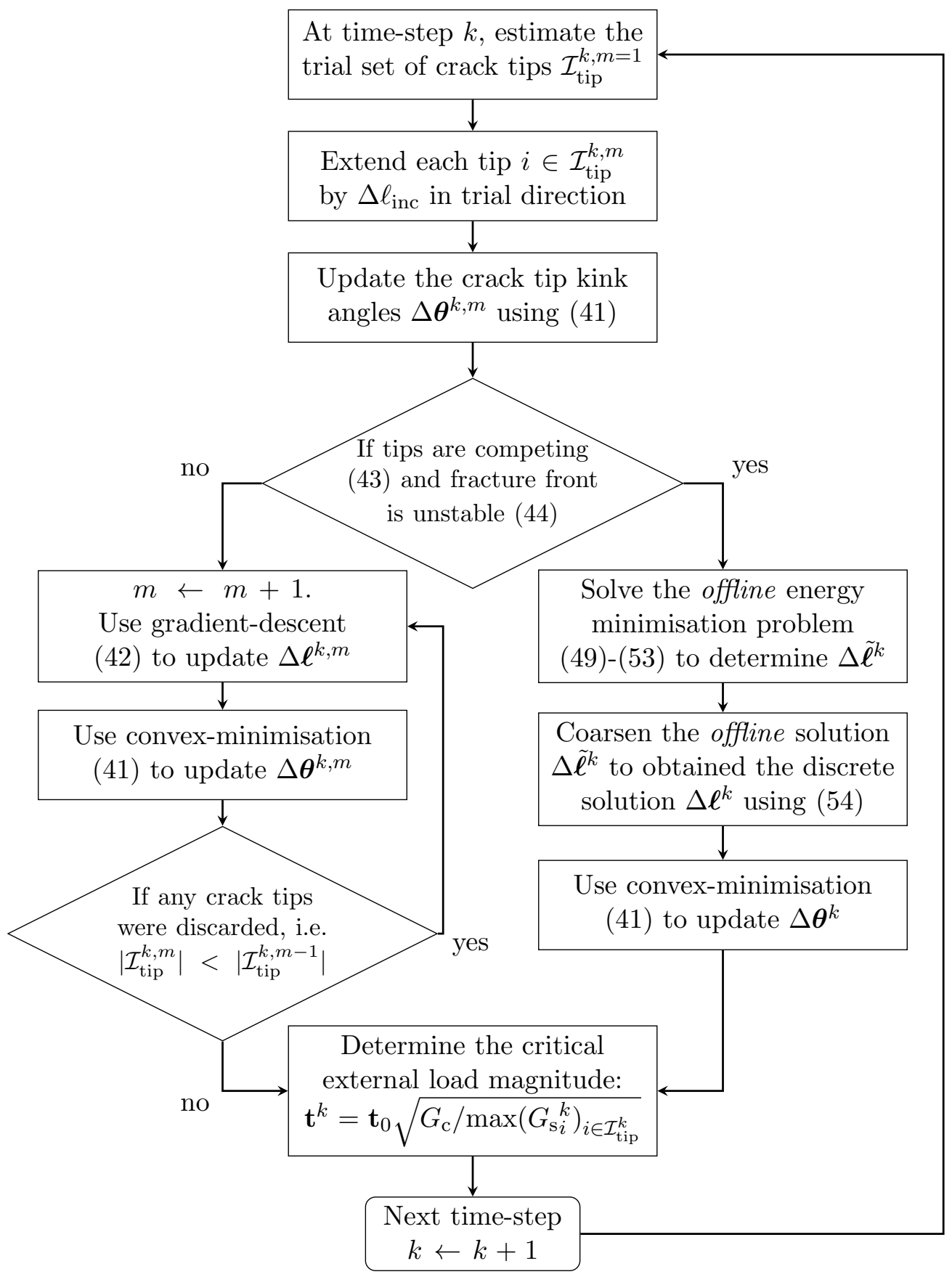

Figure 4: Flowchart of the discrete solution algorithm. 


\subsection{Verification of solution approach}

The discrete single-trial gradient-based energy minimisation approach (Section 3.3) and the multi-trial offline energy minimisation approach (Section 4.2) are assessed by solving several fabricated test cases. The fundamental assumption in all test cases is that the energy function $\tilde{\mathcal{E}}\left(\Delta \boldsymbol{\ell}^{k}, \Delta \boldsymbol{\theta}^{k}\right)$ is convex with respect to $\Delta \boldsymbol{\theta}^{k} \in \mathbb{R}_{(-\pi, \pi)}^{n_{\mathrm{tip}}}$ (refer to (40)). In other words, it is assumed that a unique stationary point of $\tilde{\mathcal{E}}\left(\Delta \boldsymbol{\ell}^{k}, \Delta \boldsymbol{\theta}^{k}\right)$ can be found with respect to $\Delta \boldsymbol{\theta}^{k}$ such that the energy will be minimised for a given $\Delta \boldsymbol{\ell}^{k}$. In turn, the test cases can be simplified to only consider $\Delta \boldsymbol{\ell}^{k}$ since the solution to $\Delta \boldsymbol{\theta}^{k}$ will be obtained implicitly. Moreover, it is sufficient to suppose a quadratic energy function as this is enough for consistency with $\mathcal{E}\left(\Delta \boldsymbol{\ell}^{k}, \Delta \boldsymbol{\theta}^{k}\right)$ and, hence, for testing the viability of the two solution approaches. Thus, the energy function is assumed to take the form:

$$
\mathcal{E}(\ell)=\mathcal{E}_{0}-\left(G_{\mathrm{s} i}-G_{\mathrm{c}}\right) \ell_{i}-\frac{1}{2} H_{\mathrm{s} i j} \ell_{i} \ell_{j} \quad \text { where } i, j \in \mathcal{I}_{\text {tip }}^{n_{\mathrm{tip}}}
$$

The aim of this verification study is to show under what properties of $\mathcal{E}(\ell)$ the two energy minimisation approaches can reliably capture the critical fracture paths for fixed-length crack tip incrementation. We consider 4 fabricated benchmark problems: competing/non-competing crack growth for convex/non-convex behaviours of $\mathcal{E}(\ell)$. Each of the 4 cases assesses the solution convergence (or non-convergence) for 3, 6 and 12 crack tip problems. The numerical results that are to follow shortly correspond to the worstcase results that could be obtained from multiple randomly generated cases. The Matlab codes that produce the results are included as supplementary material in Section 8 .

For the sake of brevity we use the term discrete in the context of the gradient-based energy minimisation approach to denote that the method assumes fixed-length crack tip extensions and that a single trial solution of uniformly extending all tips is used. In principle, we apply the solution method described in Section 4.2. The term offline will denote a gradient-based energy minimisation approach that is subjected to the constraint of a fixed-unit of total fracture length advance per time-step. In addition, a line-search routine will be used to find locally optimal solutions along convex parts of the energy function. Moreover, multiple trial solutions will be attempted, e.g. extending each crack tip as an individual initial solution. Finally, the offline approach will involve coarsening (54) of the solution to obtained the best representation of fixed-length crack tip extensions. In principle, we apply the solution method described in Section 3.3 .

The discrete and the offline solution methods are benchmarked against the so-called standard approach, which is very similar to the offline approach except that no coarsening of the minimum energy solution is performed after each time-step. In other words, the solutions obtained by the standard gradient-based method serve as reference solutions against which the discrete and the offline solution variants are compared.

In the following benchmark cases, cracks are allowed to grow until the total crack length 
reaches $\Delta a_{\text {tot }}=1$. Then, the discrepancy in the final crack tip positions relative to the reference solution is computted and plotted against different fracture extension lengths per time-step. The results for different properties of $\mathcal{E}(\ell)$ are given in Figures 5

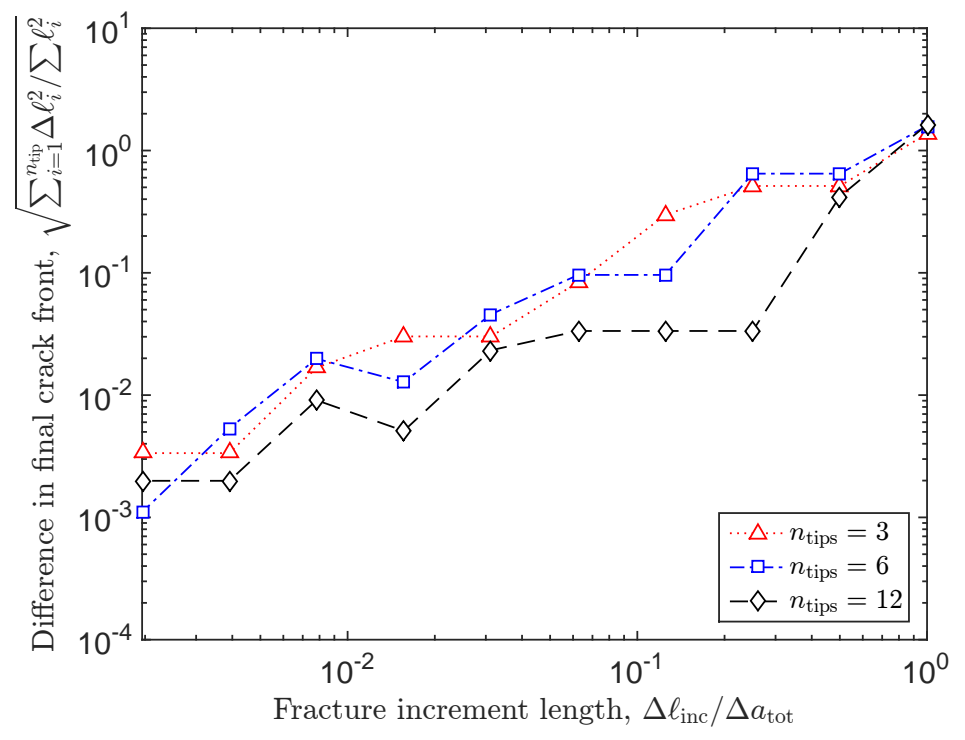

Figure 5: Non-competing crack growth and a convex energy function. Convergence of the crack tip paths to the same fracture front as obtained by standard gradient-based solution method and its discrete variant. The discrete approach is robust.

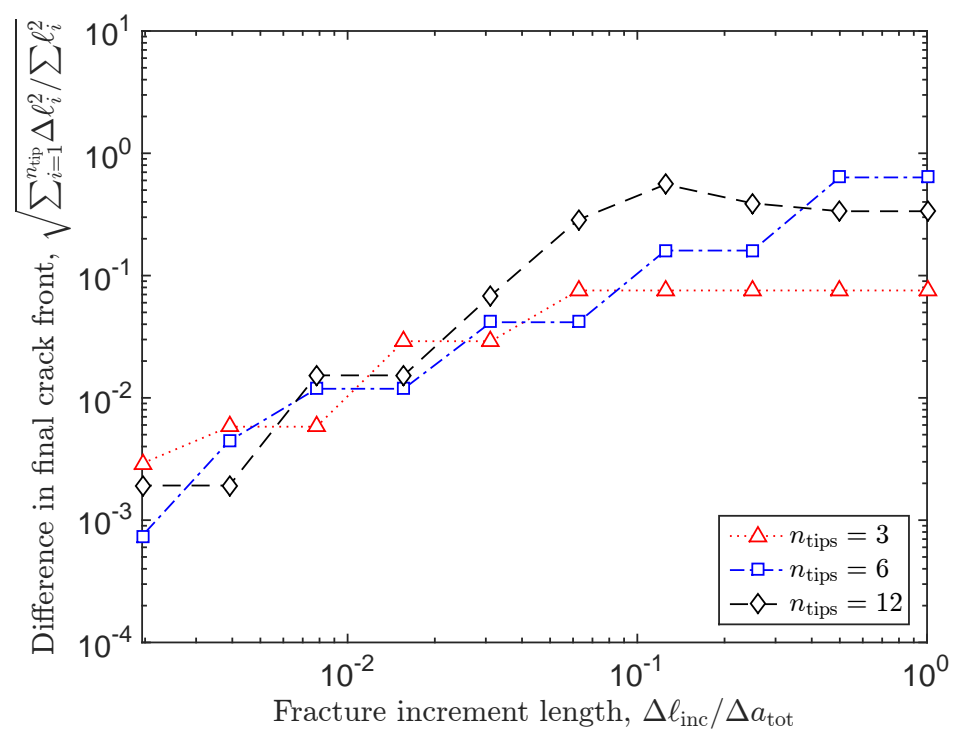

Figure 6: Non-competing crack growth and a non-convex energy function. Convergence of the crack tip paths to the same fracture front as obtained by standard gradientbased solution method and its discrete variant. The discrete approach is robust. 


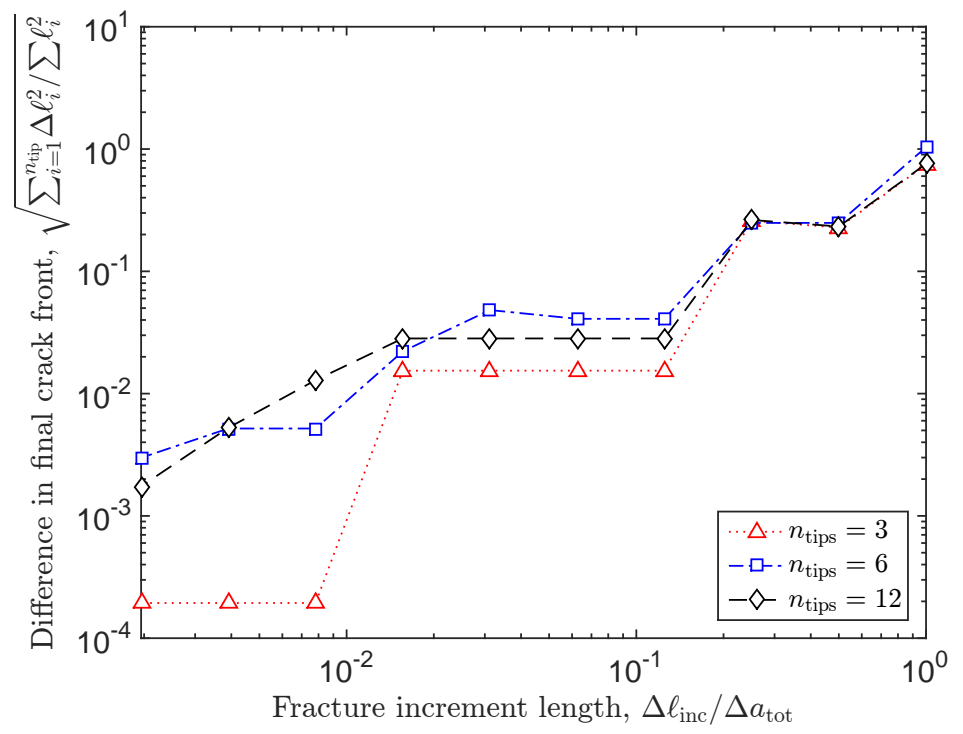

Figure 7: Competing crack growth and a convex energy function. Convergence of the crack tip paths to the same fracture front as obtained by standard gradient-based solution method and its discrete variant. The discrete approach is robust.

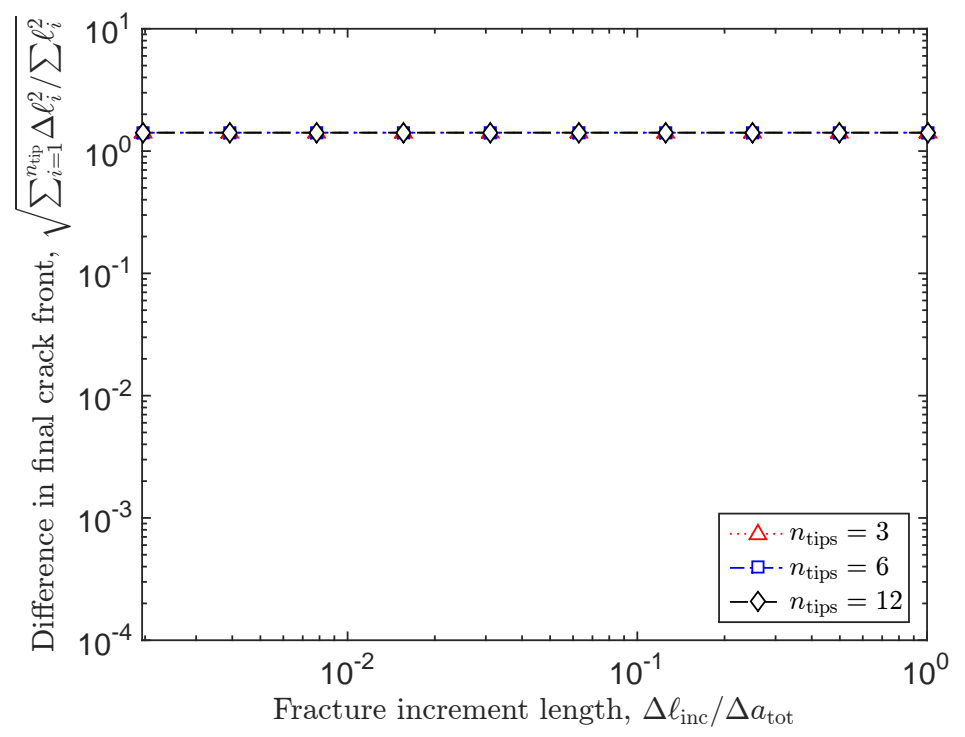

Figure 8: Competing crack growth and a concave energy function. No convergence of the crack tip paths towards the same solution as obtained by standard gradient-based solution method and its discrete variant. The discrete approach is not robust. 


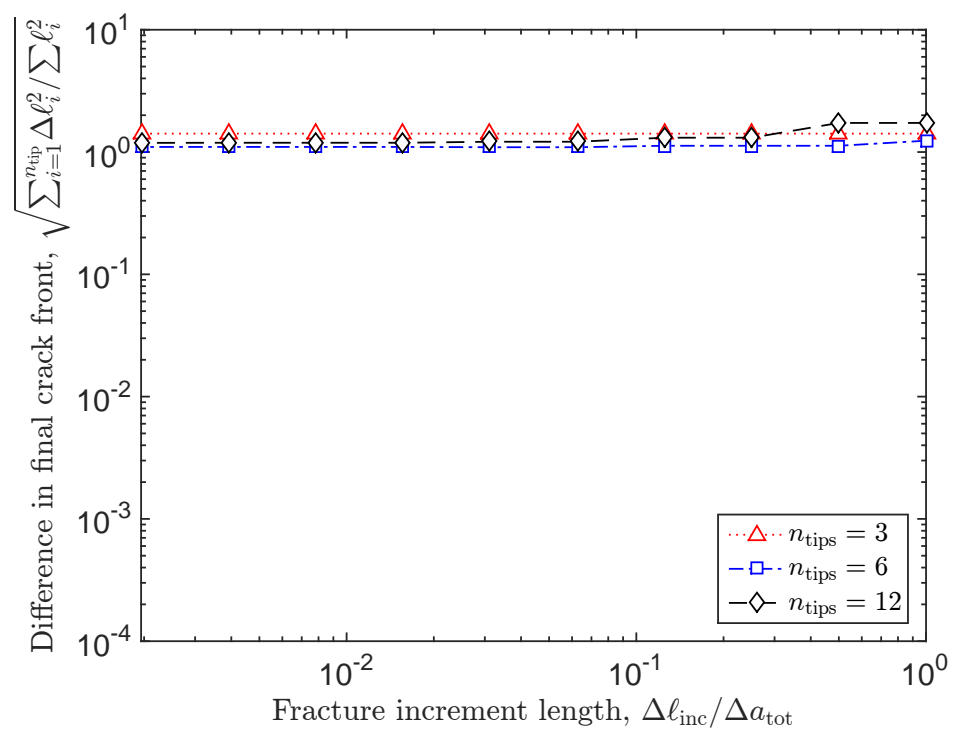

Figure 9: Competing crack growth and a non-convex energy function. No convergence of the crack tip paths to the same fracture front as obtained by standard gradient-based solution method and its discrete variant. The discrete approach is not robust. Figure 10 shows the results for the same test cases but applying the offline solution approach.

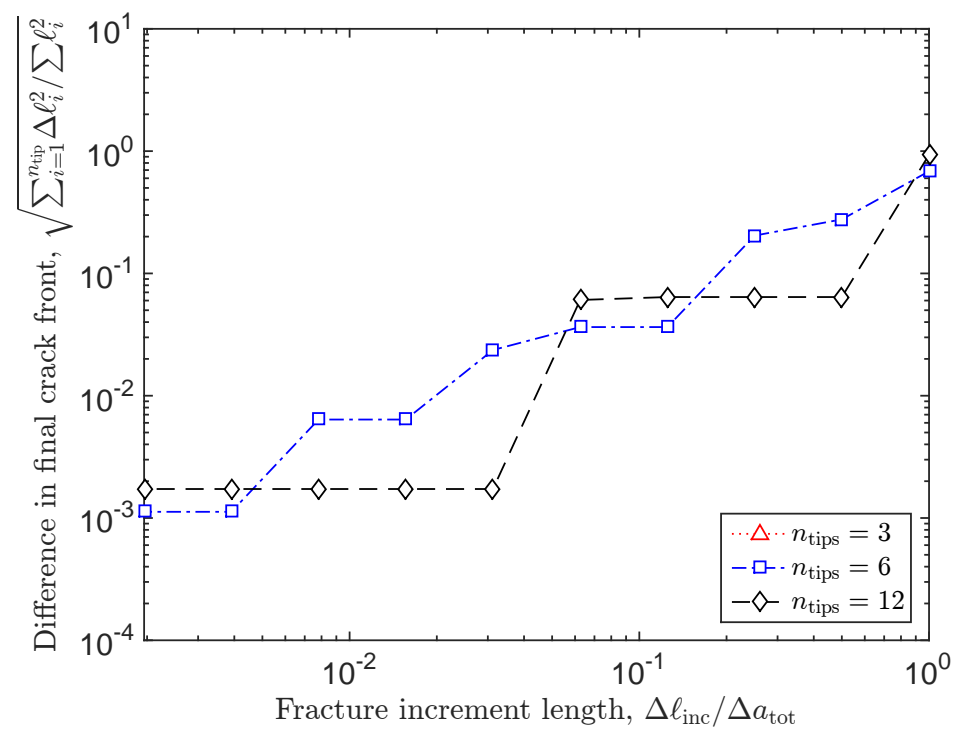

Figure 10: Competing crack growth and a non-convex energy function. Convergence of the crack tip paths to the same fracture front as obtained by standard gradient-based solution method and the proposed offline energy minimisation approach. (Note that the results for $\mathrm{n}_{\text {tip }}=3$ do not show since the solutions by the two methods are the same.) 
It can be observed from the preceding benchmark cases that the discrete gradient-based solution approach (Section 3.3) is robust in cases of non-competing crack growth. Moreover, the method works for competing crack growth provided the energy function is convex (e.g. Figure 7). On the other hand, the method is not robust when two conditions are met: competing crack growth and a non-convex (or concave) energy function (e.g. Figures 8 and 9). The method is not robust because in such cases there can exist multiple locally optimal solutions such that the converged solution can depend on the initial solution. In the case of competing crack growth and a non-convex energy function, a multi-trial gradient-based solution strategy needs to be used for its robustness. Within the discrete framework of fixed-length crack tip incrementation, the proposed offline solution approach (Section 4.2 has been shown to be able to resolve the non-convex case (e.g. Figure 10) $\left.\right|^{2}$ Thus, our complete strategy for solving general multi-crack growth problems, which can be reviewed in Figure 4 , has been shown to be adequate.

\section{Discretisation}

The outline of the extended finite element method (XFEM) is attached in Appendix A Here, we direct our attention to the computations of the energy release rates via the algebraic differentiation of the discrete potential energy as obtained through XFEM.

\subsection{Discrete energy release rates}

The potential energy of a discrete mechanical system can be generally written as:

$$
\Pi=\frac{1}{2} \mathbf{q}^{\mathrm{T}} \mathbf{K} \mathbf{q}-\mathbf{q}^{\mathrm{T}} \mathbf{f}
$$

where $\mathbf{q}, \mathbf{K}$, and $\mathbf{f}$ are, respectively, the generalised displacements vector, the stiffness matrix, and the nodal force vector. The so-called rotational energy release rate (8) of a crack extension $\Delta \ell_{i}$ is computed based on the stiffness derivative approach [23, 20]:

$$
\begin{aligned}
G_{\theta i} & =-\frac{1}{2} \mathbf{q}^{\mathrm{T}} \delta_{i} \mathbf{K q}+\mathbf{q}^{\mathrm{T}} \delta_{i} \mathbf{f}-\delta_{i} \mathbf{q}^{\mathrm{T}}(\mathbf{K q}-\mathbf{f}) \\
G_{\theta i} & =-\frac{1}{2} \mathbf{q}^{\mathrm{T}} \delta_{i} \mathbf{K q}+\mathbf{q}^{\mathrm{T}} \delta_{i} \mathbf{f}
\end{aligned}
$$

where for the sake of brevity $\delta_{i}$ is used to denote a derivative with respect to the free variable; in this case, $\theta_{i}$. Note that the last term in (57) vanishes due to the assumed

\footnotetext{
${ }^{2}$ Note that results for the cases of competing crack growth and a concave energy function have not been presented because the solutions by the standard and the offline strategies are always identical.
} 
equilibrium of the discrete system, i.e. $\mathbf{K q}=\mathbf{f}$. The rates of the rotational energy release rates for a multiply cracked solid can be computed similarly:

$$
\begin{aligned}
& H_{\theta i j}=-\left(\frac{1}{2} \mathbf{q}^{\mathrm{T}} \delta_{i j}^{2} \mathbf{K q}-\mathbf{q}^{\mathrm{T}} \delta_{i j}^{2} \mathbf{f}\right)-\delta_{j} \mathbf{q}^{\mathrm{T}}\left(\delta_{i} \mathbf{K q}-\delta_{i} \mathbf{f}\right) \\
& H_{\theta i j}=-\left(\frac{1}{2} \mathbf{q}^{\mathrm{T}} \delta_{i j}^{2} \mathbf{K q}-\mathbf{q}^{\mathrm{T}} \delta_{i j}^{2} \mathbf{f}\right)+\left(\delta_{j} \mathbf{K} \mathbf{q}-\delta_{j} \mathbf{f}\right)^{\mathrm{T}} \mathbf{K}^{-1}\left(\delta_{i} \mathbf{K} \mathbf{q}-\delta_{i} \mathbf{f}\right)
\end{aligned}
$$

where in $(59), \delta_{j} \mathbf{q}$ is determined from the condition that the variations of the equilibrium equations must be zero, i.e. $\delta_{i}(\mathbf{K q}+\mathbf{f})=\mathbf{0}$. The force variations $\delta_{i} \mathbf{f}$ and $\delta_{i j}^{2} \mathbf{f}$ need only be accounted for if the applied loads act on the virtual crack rotations, such as in the case of crack surface tractions and/or body-type loads. In (60), the first term containing the second order derivatives captures the local interaction between the rotations of different crack increments; on the other hand, the second term encompassing the products of first order variations represents the remote interaction. Concerning the second order crossdifferentials $\delta_{i j}(\cdot)$ where $i \neq j$, the cross derivatives are zero unless there is a strong geometrical coupling between the different virtual crack rotations, e.g. when crack tips are sufficiently close. On the other hand, the self-interaction $\delta_{i i}^{2}(\cdot)$ always exists.

Concerning the second order variations $\delta_{i j}^{2}(\cdot)$ it will be considered for simplicity's sake that the fracture configuration at hand is one where the crack extensions are sufficiently far apart such that the cross-variations do not arise, i.e. $\delta_{i j}^{2}(\cdot)=0$ for $i \neq j$. However, when the local-interactions do arise (eventually), say for a pair of nearby crack tips, the tips tend to already be too close for practical handling and so a crack intersection is forced instead. Note that the remote coupling between the different rates of rotational energy release rates $H_{\theta i j}$ when $i \neq j$ is retained by the products of the first order variations that appear as the second term of 60. For convenience, the discrete energy release rates and the rates of the energy release rates can be simplified to read:

$$
\begin{aligned}
G_{i} & =-\frac{1}{2} \mathbf{q}^{\mathrm{T}} \delta_{i} \mathbf{K q}+\mathbf{q}^{\mathrm{T}} \delta_{i} \mathbf{f} \\
H_{i j} & =-\left(\frac{1}{2} \mathbf{q}^{\mathrm{T}} \delta_{i i}^{2} \mathbf{K q}-\mathbf{q}^{\mathrm{T}} \delta_{i i}^{2} \mathbf{f}\right)+\left(\delta_{j} \mathbf{K q}-\delta_{j} \mathbf{f}\right)^{\mathrm{T}} \mathbf{K}^{-1}\left(\delta_{i} \mathbf{K} \mathbf{q}-\delta_{i} \mathbf{f}\right)
\end{aligned}
$$

where in 62 only the self interaction $\delta_{i i}^{2}(\cdot)$ needs to be resolved. Henceforth, the subscripts in $\delta_{i}$ and $\delta_{i i}^{2}$ can be omitted, i.e. we will use $\delta$ and $\delta^{2}$ respectively. The global stiffness matrix $\mathbf{K}$ can be obtained by summing the element-level stiffness matrices:

$$
\begin{aligned}
& \mathbf{K}=\sum_{i=1}^{n_{\mathrm{el}}} \mathbf{K}_{\mathrm{el}} \\
& \mathbf{K}=\sum_{i=1}^{n_{\mathrm{el}}} \int_{\bar{\Omega}_{\mathrm{el}}} \mathbf{B}^{\mathrm{T}} \mathbf{C B} \operatorname{det}(\mathbf{J}) \mathrm{d} \xi \mathrm{d} \eta
\end{aligned}
$$


where $n_{\mathrm{el}}$ is the number of elements, $\bar{\Omega}_{\mathrm{el}}$ is the element domain in the parametric coordinate space $(\xi, \eta), \mathbf{B}$ is a strain operator matrix, $\mathbf{C}$ is the constitutive matrix and $\mathbf{J}$ is the Jacobian of the mapping between the parametric and the Cartesian $(x, y)$ spaces.

\subsection{Stiffness and force derivatives}

The first and second order variations of the element-level stiffness matrix $\mathbf{K}_{\mathrm{el}}$ are:

$$
\begin{aligned}
\delta \mathbf{K}_{\mathrm{el}} & =\int_{\Omega_{\mathrm{el}}}\left(\delta \mathbf{B}^{\mathrm{T}} \mathbf{C B}+\mathbf{B}^{\mathrm{T}} \mathbf{C} \delta \mathbf{B}\right) \operatorname{det}(\mathbf{J}) \mathrm{d} \xi \mathrm{d} \eta \\
& +\int_{\Omega_{\mathrm{el}}} \mathbf{B}^{\mathrm{T}} \mathbf{C B} \delta \operatorname{det}(\mathbf{J}) \mathrm{d} \xi \mathrm{d} \eta \\
\delta^{2} \mathbf{K}_{\mathrm{el}} & =\int_{\Omega_{\mathrm{el}}}\left(\delta^{2} \mathbf{B}^{\mathrm{T}} \mathbf{C B}+2 \delta \mathbf{B}^{\mathrm{T}} \mathbf{C} \delta \mathbf{B}+\mathbf{B}^{\mathrm{T}} \mathbf{C} \delta^{2} \mathbf{B}\right) \operatorname{det}(\mathbf{J}) \mathrm{d} \xi \mathrm{d} \eta \\
& +\int_{\Omega_{\mathrm{el}}} 2\left(\delta \mathbf{B}^{\mathrm{T}} \mathbf{C B}+\mathbf{B}^{\mathrm{T}} \mathbf{C} \delta \mathbf{B}\right) \delta \operatorname{det}(\mathbf{J}) \mathrm{d} \xi \mathrm{d} \eta \\
& +\int_{\Omega_{\mathrm{el}}} \mathbf{B}^{\mathrm{T}} \mathbf{C B} \delta^{2} \operatorname{det}(\mathbf{J}) \mathrm{d} \xi \mathrm{d} \eta
\end{aligned}
$$

The global force vector due to the local surface tractions, e.g. pressure $p$, is obtained by summing the element level contributions of the elements that are cut by the cracks:

$$
\begin{aligned}
& \mathbf{f}=\sum_{i=1}^{n_{\mathrm{el}}^{\mathrm{cut}}} \mathbf{f}_{\mathrm{el}} \\
& \mathbf{f}=\sum_{i=1}^{n_{\mathrm{el}}^{\text {cut }}} \int_{\Gamma_{\mathrm{c}}^{i}} \llbracket \mathbf{N} \rrbracket^{\mathrm{T}} \mathbf{n}^{-} p \frac{\mathrm{d} l}{\mathrm{~d} \zeta} \mathrm{d} \zeta
\end{aligned}
$$

where $\llbracket \mathbf{N} \rrbracket$ denotes a jump in the displacement-like matrix $\mathbf{N}$ across the fracture interface, $\frac{\mathrm{d} l}{\mathrm{~d} \zeta}$ is the Jacobian of the mapping between the parametric $(\zeta)$ and the physical coordinate $(l)$ that is on the part of the fracture surface that cuts a particular element. The first and second differentials of the force vector $\mathbf{f}_{\mathrm{el}}$ can be generally computed as follows:

$$
\begin{aligned}
\delta \mathbf{f}_{\mathrm{el}} & =\int_{\Gamma_{\mathrm{c}}}\left(\delta \llbracket \mathbf{N} \rrbracket^{\mathrm{T}} \mathbf{T}+\llbracket \mathbf{N} \rrbracket^{\mathrm{T}} \delta \mathbf{T}\right) \mathbf{p} \frac{\mathrm{d} l}{\mathrm{~d} \zeta} \mathrm{d} \zeta+\int_{\Gamma_{\mathrm{c}}} \llbracket \mathbf{N} \rrbracket^{\mathrm{T}} \mathbf{T} \mathbf{p} \frac{\mathrm{d}}{\mathrm{d} \zeta}(\delta l) \mathrm{d} \zeta \\
\delta^{2} \mathbf{f}_{\mathrm{el}} & =\int_{\Gamma_{\mathrm{c}}}\left(\delta^{2} \llbracket \mathbf{N} \rrbracket^{\mathrm{T}} \mathbf{T}+2 \delta \llbracket \mathbf{N} \rrbracket^{\mathrm{T}} \delta \mathbf{T}+\llbracket \mathbf{N} \rrbracket^{\mathrm{T}} \delta^{2} \mathbf{T}\right) \mathbf{p} \frac{\mathrm{d} l}{\mathrm{~d} \zeta} \mathrm{d} \zeta \\
& +\int_{\Gamma_{\mathrm{c}}}\left(\delta \llbracket \mathbf{N} \rrbracket^{\mathrm{T}} \mathbf{T}+\llbracket \mathbf{N} \rrbracket^{\mathrm{T}} \delta \mathbf{T}\right) \mathbf{p} \frac{\mathrm{d}}{\mathrm{d} \zeta}(\delta l) \mathrm{d} \zeta+\int_{\Gamma_{\mathrm{c}}} \llbracket \mathbf{N} \rrbracket^{\mathrm{T}} \mathbf{T} \mathbf{p} \frac{\mathrm{d}}{\mathrm{d} \zeta}\left(\delta^{2} l\right) \mathrm{d} \zeta
\end{aligned}
$$

where the crack surface tractions $\mathbf{p}=[0, p]^{\mathrm{T}}$ are assumed to be independent of the crack extension angle $\theta . \mathbf{T}(\theta)=\left[\begin{array}{cc}\cos \theta & -\sin \theta \\ \sin \theta & \cos \theta\end{array}\right]$ is a local-to-global transformation matrix. The detailed element-level derivative computations are provided in Appendix A.3. 


\subsection{Computational strategy}

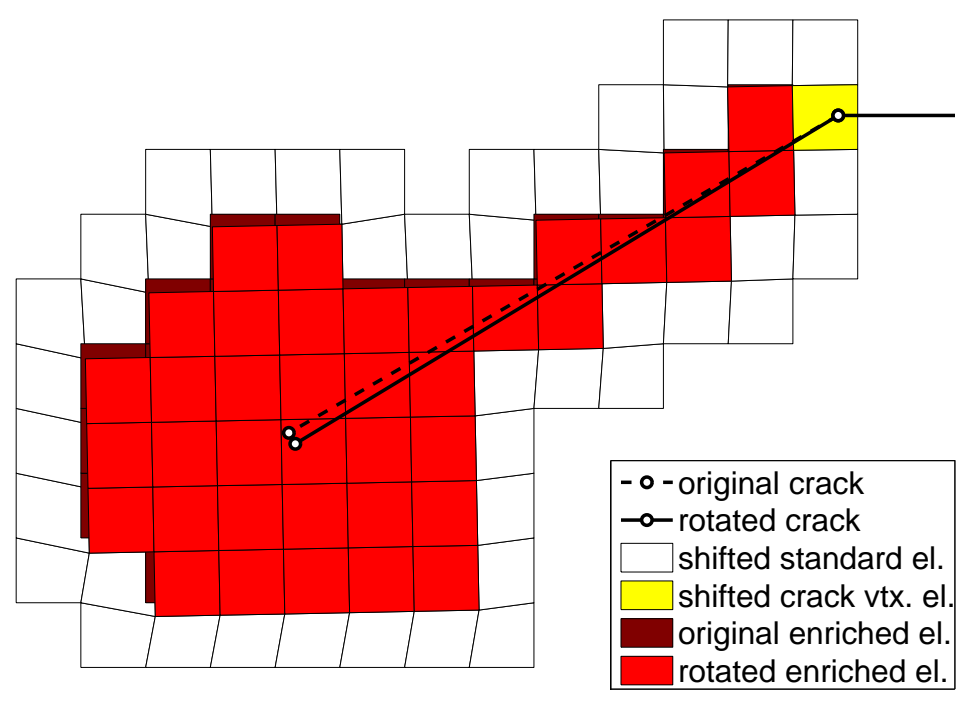

Figure 11: Virtual perturbation of the mesh in the crack tip vicinity for computing the derivatives of the stiffness matrix and the force vector. The enriched elements (in red) translate coherently with the rotation of the crack tip segment; as such, they experience no change in shape but only a rigid rotation. The ring of unenriched elements (in white), which surrounds the patch of enriched elements, has its outer boundary fixed to the rest of the mesh whereas its inner boundary conformally follows the patch of enriched elements; as such, the surrounding elements experience shape distortion.

To compute the rotational energy release rates: $G_{\theta i}$ and $H_{\theta i j}$, the derivatives of the global stiffness matrix $\mathbf{K}$ and force vector $\mathbf{f}$ are required with respect to the angular positions $\theta_{i}$ of the crack tip extensions $i, j \in \mathcal{I}_{\text {tip }}$. Similarly to the stiffness-derivative method for computing energy release rates [26, 23], we consider virtual displacements of the mesh in the vicinity of the crack tip domain. Considering the XFEM discretisation, a possible mesh perturbation surrounding a finite-length crack extension is shown in Figure 11. The derivatives of $\mathbf{K}$ and $\mathbf{f}$ are obtained by summing the element-level variations of $\mathbf{K}_{\mathrm{el}}$ and $\mathbf{f}_{\mathrm{el}}$ of the elements affected by the virtual displacements of the mesh.

It can be observed that the finite elements in the perturbed domain are subjected to two types of transformations: (1) shape distortions (2) and rigid translations. It is useful to take this into account in computing the algebraic derivatives of $\mathbf{K}_{\mathrm{el}}$ and $\mathbf{f}_{\mathrm{el}}$. For the first kind of transformation (shape distortion), the derivatives are computed using expressions: $(65),(66),(69)$ and $(70)$. Although the same approach can be applied to the second kind of transformation (rigid translation), it is unnecessarily cumbersome. Note that for an element in pure rotation the derivative of the Jacobian must vanish. 
Thus, only the derivatives of the generalised $B$-matrix are required:

$$
\begin{aligned}
\delta \mathbf{B}_{I} & =\left.\mathbf{B}_{I} \delta \mathbf{T}^{\prime}\right|_{\theta=0} \\
\delta^{2} \mathbf{B}_{I} & =\left.\mathbf{B}_{I} \delta^{2} \mathbf{T}^{\prime}\right|_{\theta=0}
\end{aligned}
$$

where $\mathbf{T}^{\prime}(\alpha)=\left[\begin{array}{cc}\cos \alpha & \sin \alpha \\ -\sin \alpha & \cos \alpha\end{array}\right]$ is the global-to-local axis transformation matrix and $\alpha$ is the rotation angle of the crack tip extension. The derivatives of $\mathbf{T}^{\prime}(\alpha)$ are: $\delta \mathbf{T}^{\prime}=\left[\begin{array}{cc}0 & 1 \\ -1 & 0\end{array}\right]$ and $\delta^{2} \mathbf{T}^{\prime}=\left[\begin{array}{cc}-1 & 0 \\ 0 & -1\end{array}\right]$. With regard to $\mathbf{f}_{\mathrm{el}}$, only the terms containing $\delta \mathbf{T}^{\prime}$ and $\delta^{2} \mathbf{T}^{\prime}$ need to be evaluated, i.e. the variations of the Jacobian and the generalised $N$-matrix are zero. In practice, the derivatives of $\mathbf{K}_{\mathrm{el}}$, can be obtained by directly manipulating the rows and columns of $\mathbf{K}_{\mathrm{el}}$; specifically, in taking (71) and $(72)$ into account we can obtain:

$$
\begin{aligned}
& \delta \mathbf{K}_{\mathrm{el} I J}=\mathbf{K}_{\mathrm{el} I J} \delta \mathbf{T}^{\prime}+\left(\mathbf{K}_{\mathrm{el} I J} \delta \mathbf{T}^{\prime}\right)^{\mathrm{T}} \\
& \delta^{2} \mathbf{K}_{\mathrm{el} I J}=2\left(\delta \mathbf{T}^{\prime \mathrm{T}} \mathbf{K}_{\mathrm{el} I J} \delta \mathbf{T}^{\prime}-\mathbf{K}_{\mathrm{el} I J}\right)
\end{aligned}
$$

Since the variations of $\mathbf{K}_{\mathrm{el}}$ and $\mathbf{f}_{\mathrm{el}}$ of enriched finite elements are computationally more expensive to determine relative to the unenriched elements, the mesh perturbation domain is chosen strategically. Specifically, we choose the domain (as depicted in Figure 11) such that the entire patch of the branch-enriched elements centred at the crack tip plus the Heaviside-enriched elements that are entirely cut by the crack extension undergo pure rotation/translation, whereas the surrounding ring of unenriched elements plus the Heaviside-enriched crack vertex element undergo shape distortion. Note that algebraic differentiation is required of the enriched crack vertex element since it experiences shape distortion. Appendix A.3 details the computational procedure within XFEM.

\section{Verification}

The verification of the minimum energy criterion is in two parts. The first part is concerned with the accuracy of the algebraically computed rotational energy release rates. The second part compares the minimum energy criterion to the maximum hoop stress criterion in terms of the incipient crack growth direction and the energy release rate. The minimum energy criterion is verified to yield a greater energy dissipation in the direction of crack growth than the solution by the maximum hoop stress criterion.

\subsection{Rotational energy release rates}

The aim of this section is to verify the computations of the rotational energy release rates: $G_{\theta}$ and $H_{\theta}=\partial G_{\theta} / \partial \theta$ within the XFEM framework. The numerical case studies assume a square plate with an edge crack (see Figure 12) subjected to two loading conditions. In case- 1 , the plate is subjected to a vertical tension, and in case- 2 the loading is only 
by internal crack pressure. In each case, the methodology consists of extending the edge crack by $\Delta \ell$ over a range of inclination angles $\theta_{\text {inc }} \in\{-75,-74, \ldots, 0\}$ of the crack tip branch. The corresponding values of $G_{\theta}$ and $\partial G_{\theta} / \partial \theta$ are computed in two ways: (1) based on the stiffness derivative approach (herein called as the algebraic solution), and (2) using numerical central finite differencing of the potential energy. The verification is carried out on several meshes and XFEM enrichment schemes, namely: enrichment of a fixed radius patch (called geometrical enrichment) and enrichment of a fixed number of elements around the crack tip (called topological enrichment). The results for the algebraically and numerically determined $G_{\theta}$ and $\partial G_{\theta} / \partial \theta$ are presented as follows.

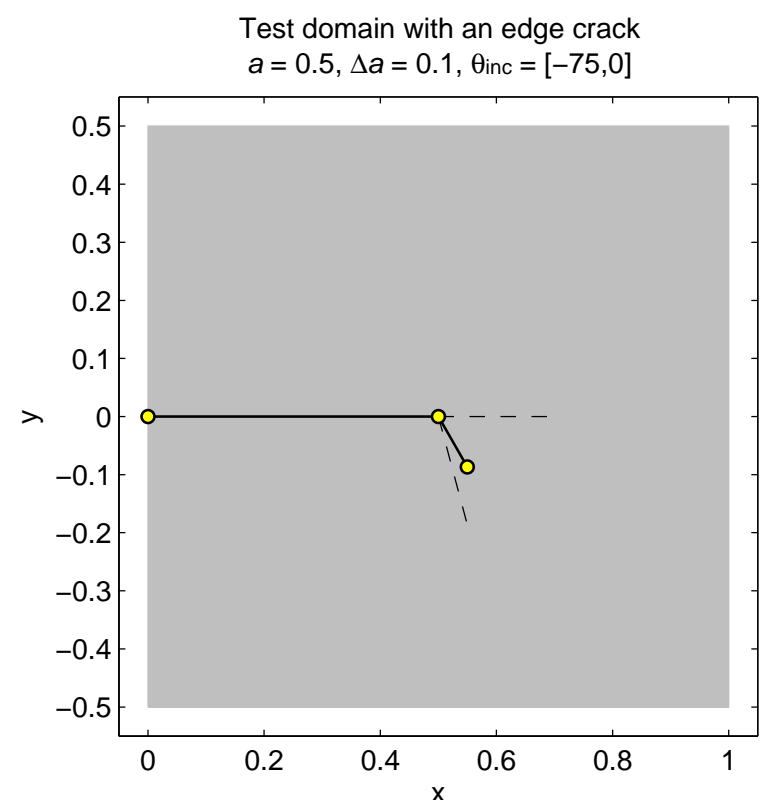

Figure 12: Test problem for assessing the accuracy of the computations of the rotational energy release rates for the crack tip extension $\Delta \ell$ at different inclination angles $\theta_{\text {inc }}$.

For test case-1, Figure 13 shows the rotational energy release rates for different crack tip increment angles as obtained on different mesh sizes and XFEM crack tip enrichment strategies. It is found that the algebraically determined $G_{\theta}$ is a much more regular function of $\theta_{\text {inc }}$ than the finite-differenced solution. Topological and geometrical variants of XFEM both yield almost indistinguishable solutions for $G_{\theta}$. Figure 16 shows the results for the rates of the rotational energy release rates $\mathrm{d} G_{\theta} / \mathrm{d} \theta$ corresponding to the different discretisations. It is found that with mesh refinement $\mathrm{d} G_{\theta} / \mathrm{d} \theta$ converges when geometrical enrichment is used and diverges in the case of topological enrichment; Figure 17 summarises these findings. Similarly, for test case-2, Figure 15 compares $G_{\theta}$, Figure $16-\mathrm{d} G_{\theta} / \mathrm{d} \theta$, and Figure 18 summarises the behaviour of the discrepancy in $\mathrm{d} G_{\theta} / \mathrm{d} \theta$ between topological and geometrical XFEM enrichment strategies.

In general, the algebraically determined $G_{\theta}$ is significantly more precise than the solution obtained by finite differencing of the potential energy. This holds irrespective of the 
XFEM enrichment strategy or mesh size. As concerns algebraically determined $\mathrm{d} G_{\theta} / \mathrm{d} \theta$, only geometrical enrichment is found to yield a convergent solution that approaches the solution obtained by finite differencing of the algebraically computed $G_{\theta}$. Unfortunately, the algebraic solution for $\mathrm{d} G_{\theta} / \mathrm{d} \theta$ diverges with mesh refinement when topological enrichment is used. Although the solution to $\mathrm{d} G_{\theta} / \mathrm{d} \theta$ (as obtained by topological XFEM) is rough, it useful nonetheless because it can approximate the gradient of $G_{\theta}$ sufficiently well for the purposes of determining the crack tip extension directions via (41).

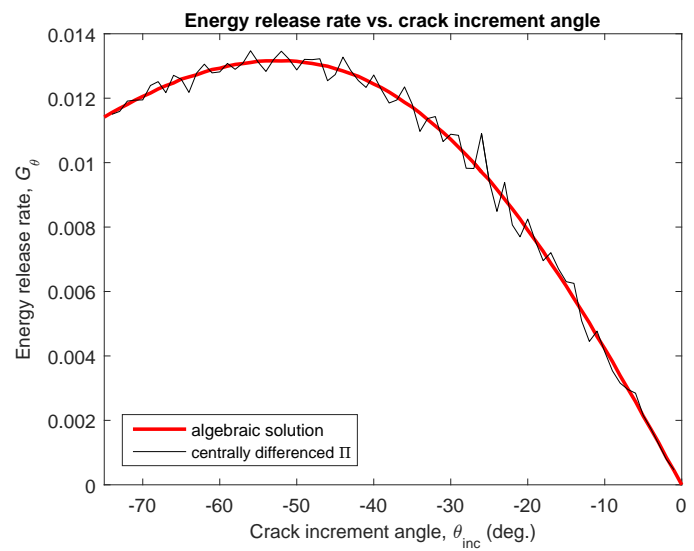

(a) $G_{\theta}$ for mesh $n_{\text {elem }}=200 \times 200$ and topological XFEM.

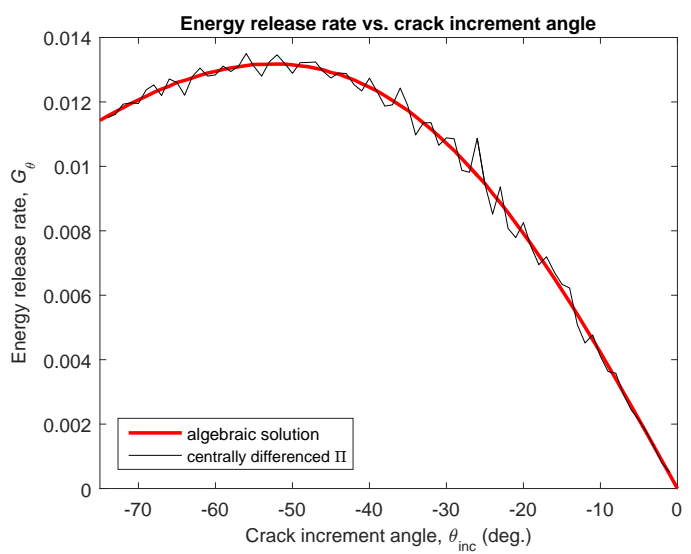

(c) $G_{\theta}$ for mesh $n_{\text {elem }}=200 \times 200$ and geometrical XFEM.

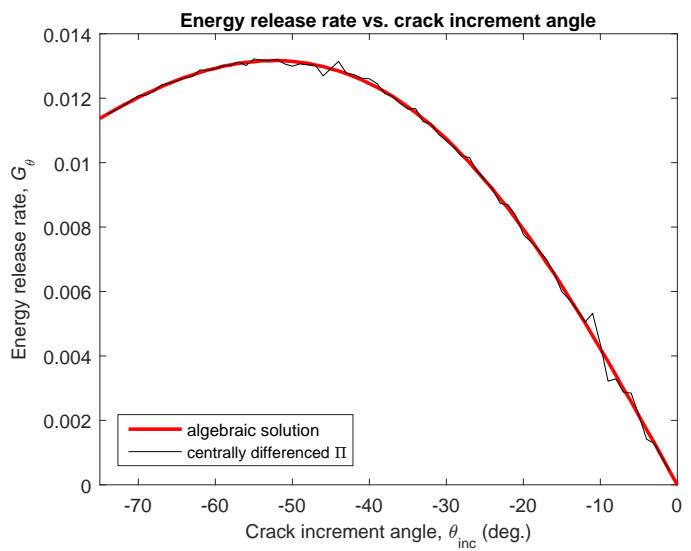

(b) $G_{\theta}$ for mesh $n_{\text {elem }}=400 \times 400$ and topological XFEM.

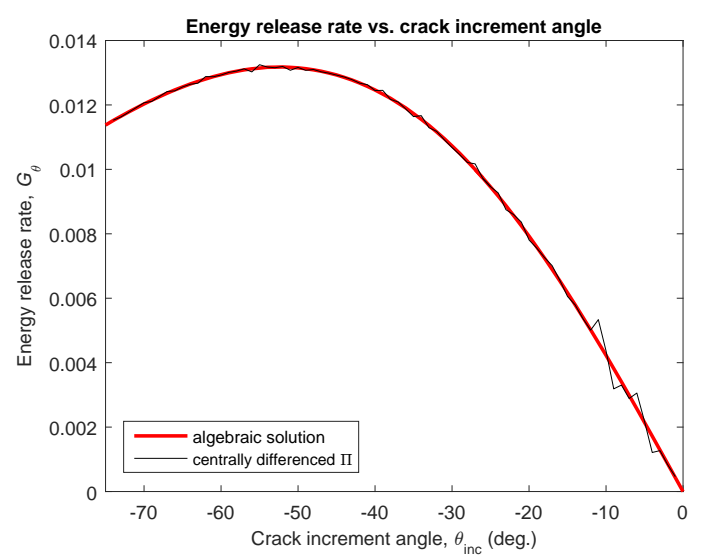

(d) $G_{\theta}$ for mesh $n_{\text {elem }}=400 \times 400$ and geometrical XFEM.

Figure 13: Comparison of the rotational energy release rate $G_{\theta}$ as computed algebraically and by finite (central) differencing of the potential energy. The test problem is a simply supported square plate with an edge crack that is subjected to vertical tension. 


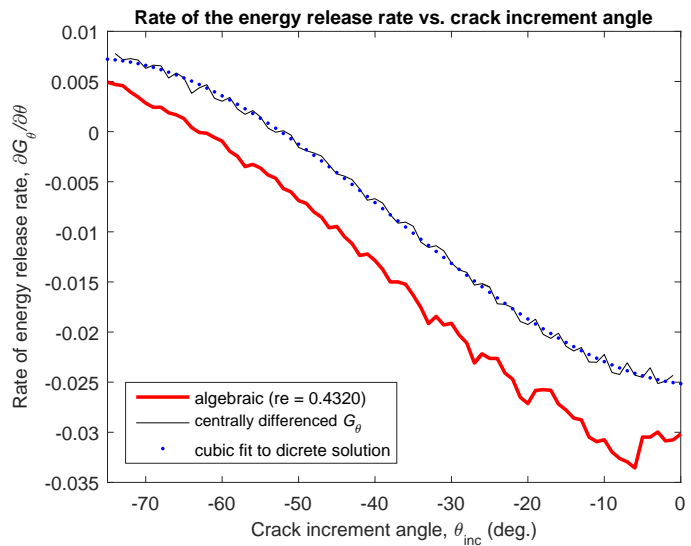

(a) $\mathrm{d} G_{\theta} / \mathrm{d} \theta$ for mesh $n_{\text {elem }}=200 \times 200$ and topological XFEM.

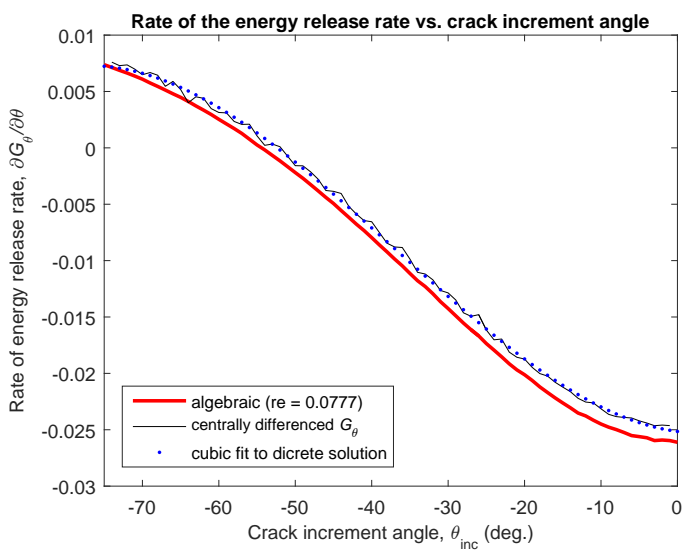

(c) $\mathrm{d} G_{\theta} / \mathrm{d} \theta$ for mesh $n_{\text {elem }}=200 \times 200$ and geometrical XFEM.

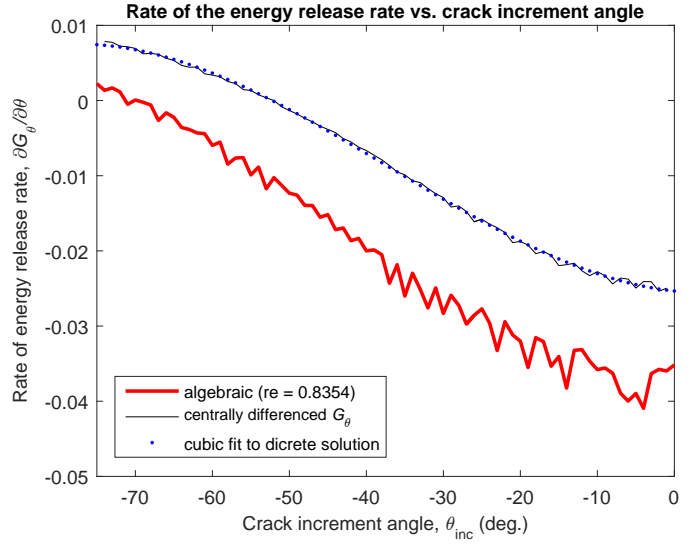

(b) $\mathrm{d} G_{\theta} / \mathrm{d} \theta$ for mesh $n_{\text {elem }}=400 \times 400$ and topological XFEM.

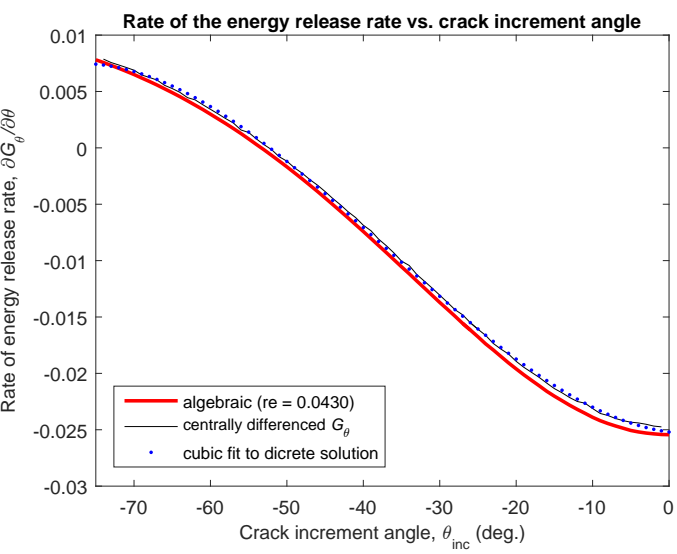

(d) $\mathrm{d} G_{\theta} / \mathrm{d} \theta$ for mesh $n_{\text {elem }}=400 \times 400$ and geometrical XFEM.

Figure 14: Comparison of the rates of rotational energy release rate $\mathrm{d} G_{\theta} / \mathrm{d} \theta$ as computed algebraically and by finite differencing of the algebraically obtained $G_{\theta}$. The test problem is a simply supported square plate with an edge crack that is subjected to vertical tension.

\subsection{Comparison to the stress criterion}

The purpose of the following numerical test cases is to compare the minimum energy and the maximum hoop stress crack propagation criteria in terms of the incipient direction of crack growth and the corresponding energy release rate for different modes of loading. Three test cases are presented. The first case considers a square domain with an initial edge crack. The square domain is subjected to displacement loading conditions at the top and bottom faces such they induce simultaneous opening (mode-I) and sliding (mode-II) loading conditions at the crack tip. The second case considers a square plate in a vertical 


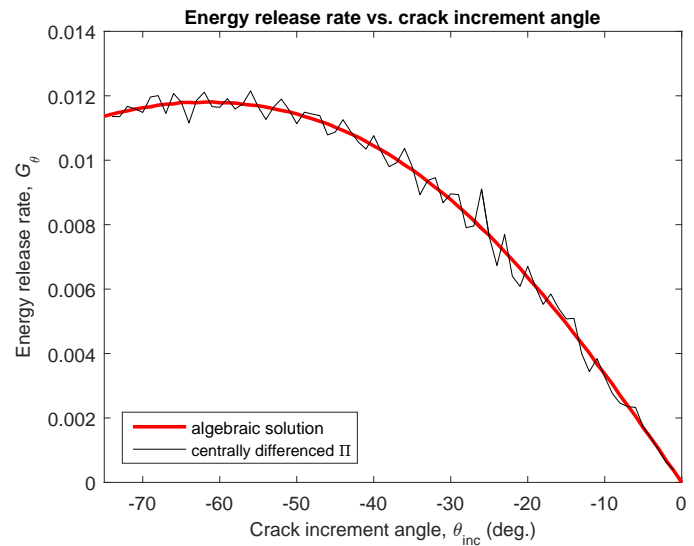

(a) $G_{\theta}$ for mesh $n_{\text {elem }}=200 \times 200$ and topological XFEM.

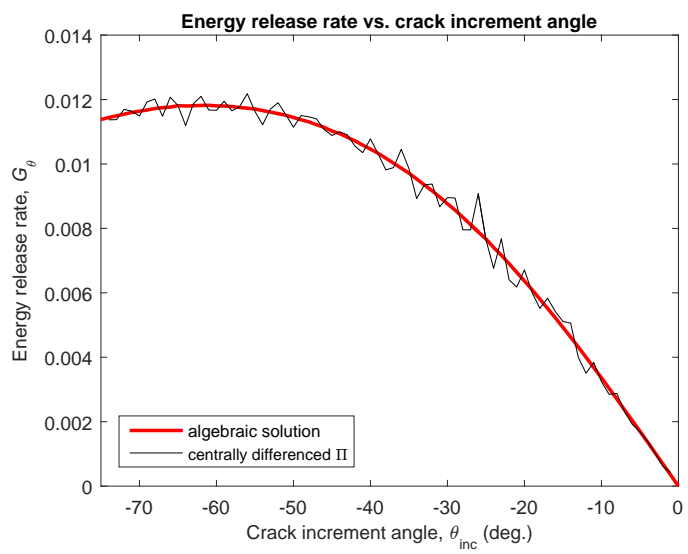

(c) $G_{\theta}$ for mesh $n_{\text {elem }}=200 \times 200$ and geometrical XFEM.

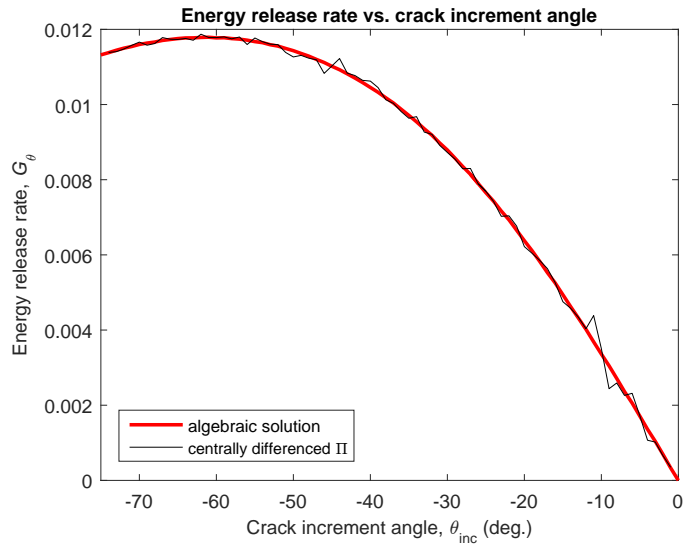

(b) $G_{\theta}$ for mesh $n_{\text {elem }}=400 \times 400$ and topological XFEM.

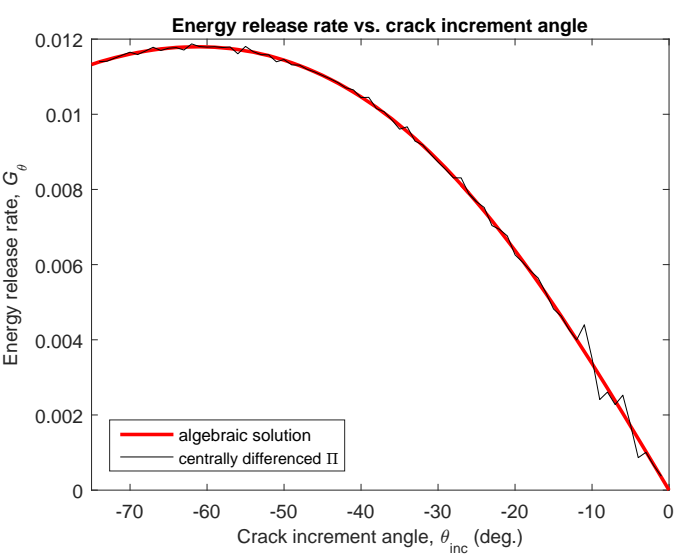

(d) $G_{\theta}$ for mesh $n_{\text {elem }}=400 \times 400$ and geometrical XFEM.

Figure 15: Comparison of the rotational energy release rate $G_{\theta}$ as computed algebraically and by finite (central) differencing of the potential energy. The test problem is a simply supported square plate with an edge crack that is under internal pressure.

tension with an initial inclined centre crack. The crack tips are subjected to a range of mixed mode-loading conditions depending on the inclination angle of the crack. The third case is just like the second except the crack is also preloaded with pressure.

The results of case- 1 , case- 2 and case- 3 are given by Figures 19,20 and 21. The subfigures for each case show the incipient fracture advance direction, the difference in the crack tip kink angle, and the relative energy release rate, as obtained by the two criteria for a range of mixed-mode loading conditions. Considering the incipient crack tip kink angles, the differences tend to increase with more significant mode-II loading. Although for smaller crack tip extension lengths these differences tend to reduce, they, however, 


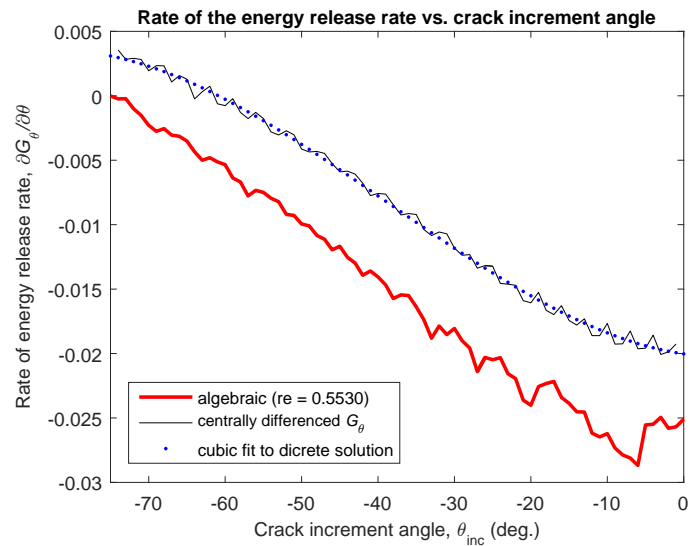

(a) $\mathrm{d} G_{\theta} / \mathrm{d} \theta$ for mesh $n_{\text {elem }}=200 \times 200$ and topological XFEM.

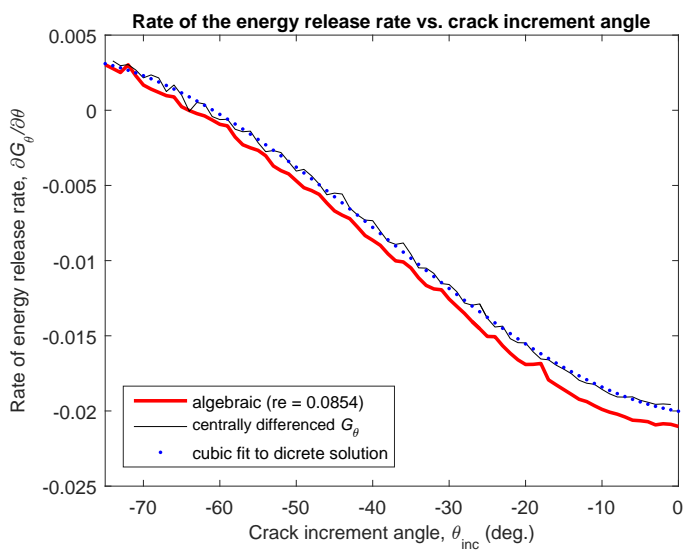

(c) $\mathrm{d} G_{\theta} / \mathrm{d} \theta$ for mesh $n_{\text {elem }}=200 \times 200$ and geometrical XFEM.

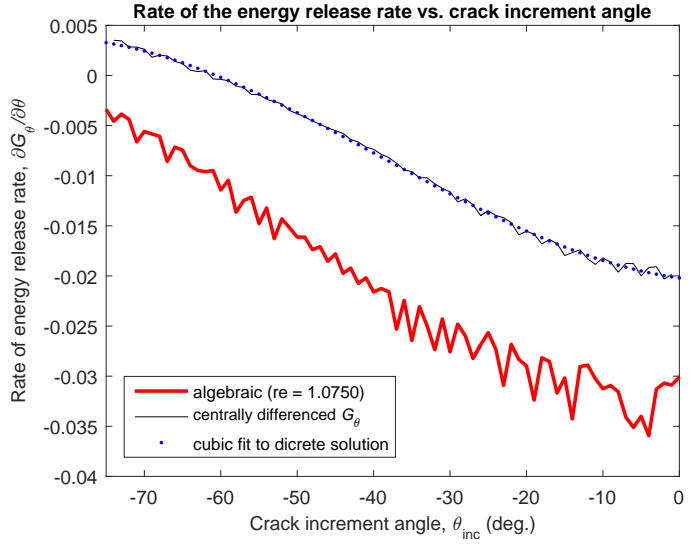

(b) $\mathrm{d} G_{\theta} / \mathrm{d} \theta$ for mesh $n_{\text {elem }}=400 \times 400$ and topological XFEM.

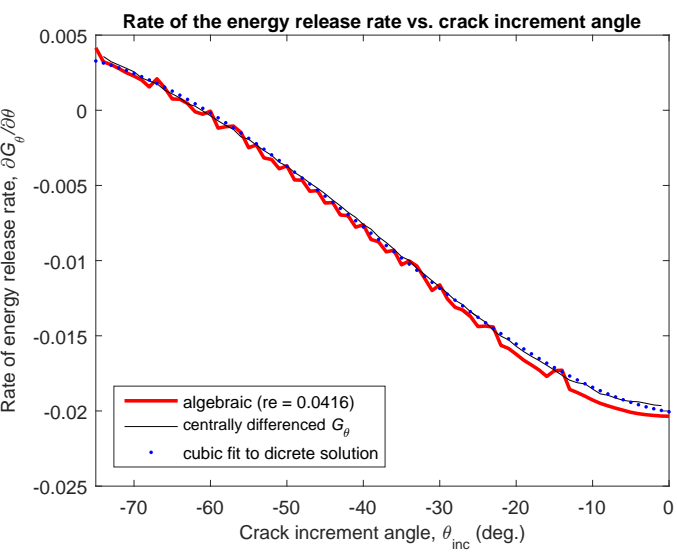

(d) $\mathrm{d} G_{\theta} / \mathrm{d} \theta$ for mesh $n_{\text {elem }}=400 \times 400$ and geometrical XFEM.

Figure 16: Comparison of the rates of rotational energy release rate $\mathrm{d} G_{\theta} / \mathrm{d} \theta$ as computed algebraically and by finite differencing of the algebraically determined $G_{\theta}$. The test problem is a simply supported square plate with an edge crack under internal pressure.

do not vanish. For crack tip kink angles larger than around $70^{\circ}$ the solutions by the two criteria depart most significantly. This is because the maximum stress criterion has a theoretical upper bound of $70.53^{\circ}$ on the absolute crack tip kind angle whereas the discrete implementation of the minimum energy criterion is not explicitly bounded and, hence, kink angles greater than $90^{\circ}$ are generally possible (e.g. see Figure 20).

In some problems the energy functional can have multiple minima. As such, it is possible for the numerical solution to converge to a local energy minimiser rather than the global one. An example is case-3 (refer to Figure 21); the Newton iterations (41) overshoot and the solution converges to a local energy minimum, i.e. a sub-optimal solution. However, 


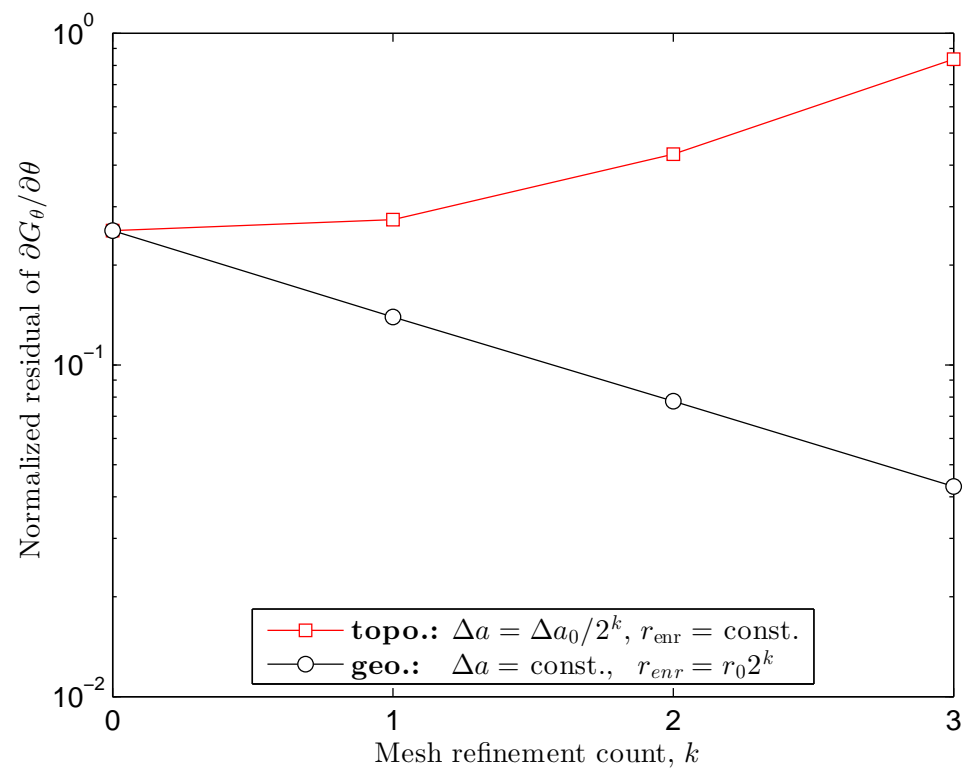

Figure 17: Discrepancy in $\mathrm{d} G_{\theta} / \mathrm{d} \theta$ between the algebraically computed solution and the best-fit solution to the finite-differencing of the algebraically determined $G_{\theta}$ for geometrical and topological XFEM enrichment schemes. The test case (case-1) assumes remote tensile loading conditions on top and bottom surfaces of the square plate.

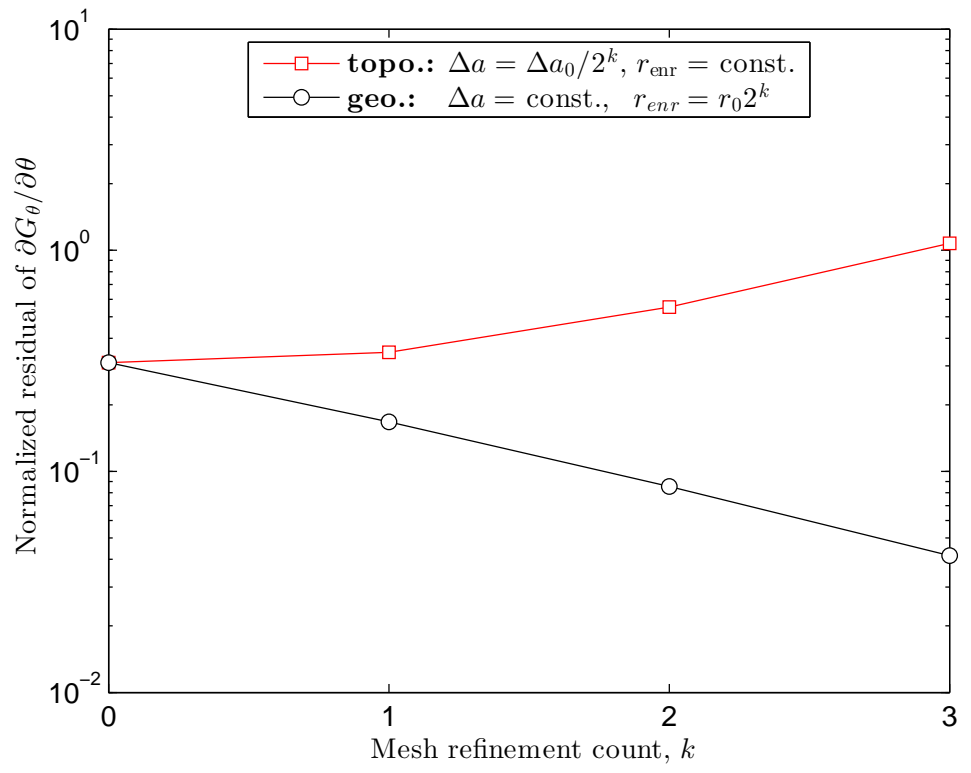

Figure 18: Discrepancy in $\mathrm{d} G_{\theta} / \mathrm{d} \theta$ between the algebraically computed solution and the best-fit solution to the finite-differencing of the algebraically determined $G_{\theta}$ for geometrical and topological XFEM enrichment schemes. The test case (case-2) assumes that the edge crack is loaded by internal crack surface pressure. 
this was observed only for the coarsest discretisation; when the discretisation was refined the solution algorithm had no trouble to converge to the globally optimal solution.
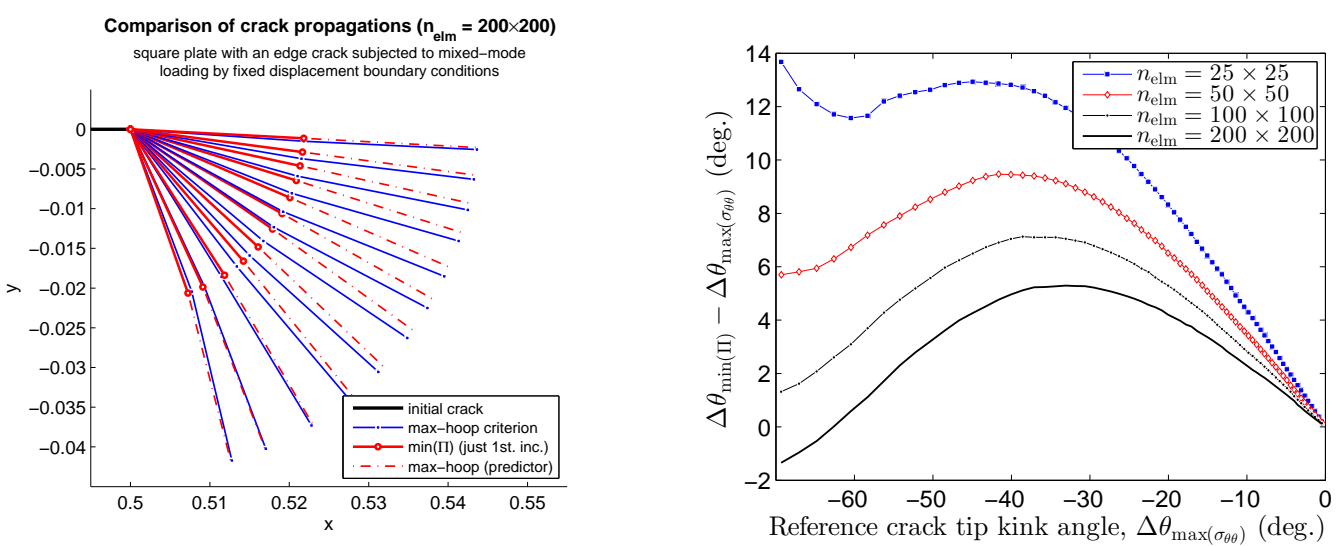

(a) Comparison of incipient crack kink angles. (b) Differences in incipient crack kink angles.

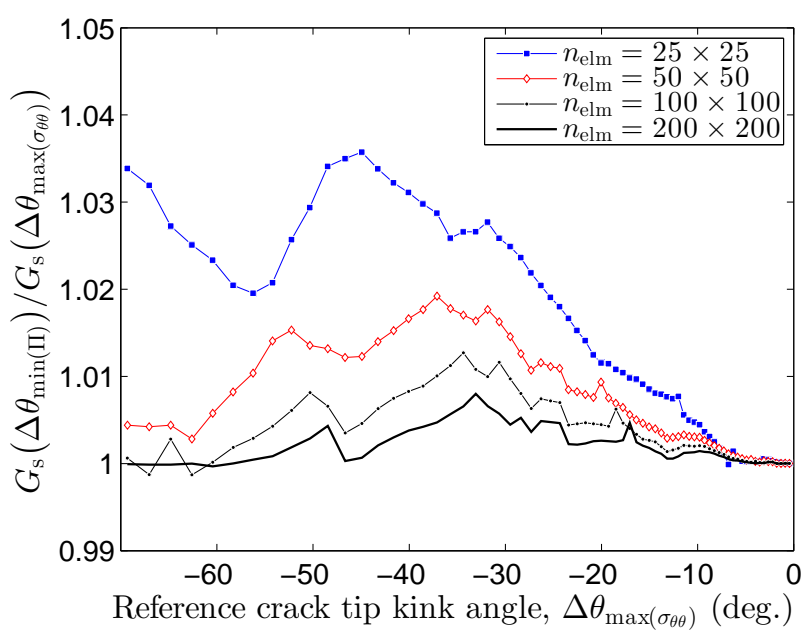

(c) Comparison of the mean energy release rates, as obtained by the minimum energy criterion relative to the maximum hoop stress criterion. The mean energy release rates are computed by differencing the potential energy between the incremented and the pre-incremented cracks (hence the reason why the plots are not so smooth).

Figure 19: Comparison of crack growth criteria for test case \#1 of a square plate with an edge crack that is subjected to mixed mode loading by prescribed displacement boundary conditions on the top and bottom edges of the plate such that they induce simultaneous opening (mode-I) and sliding (mode-II) deformations at the tip of the initial crack. 


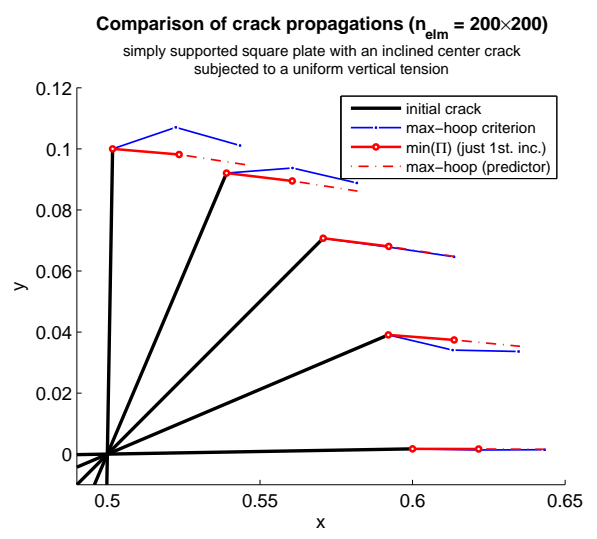

(a) Comparison of incipient crack kink angles.

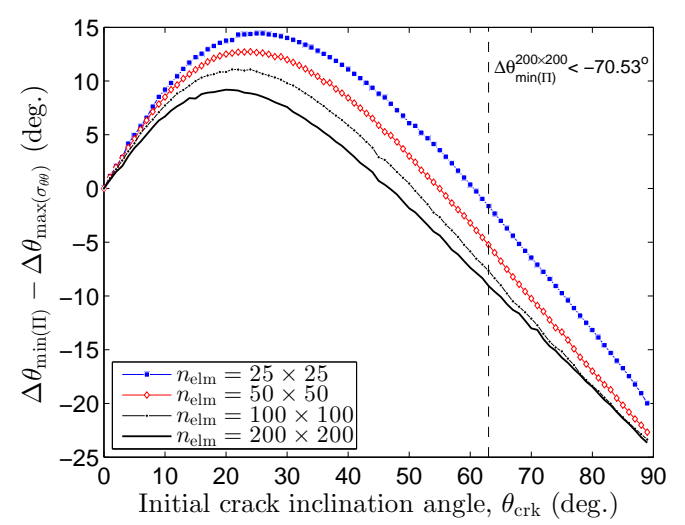

(b) Differences in incipient crack kink angles.

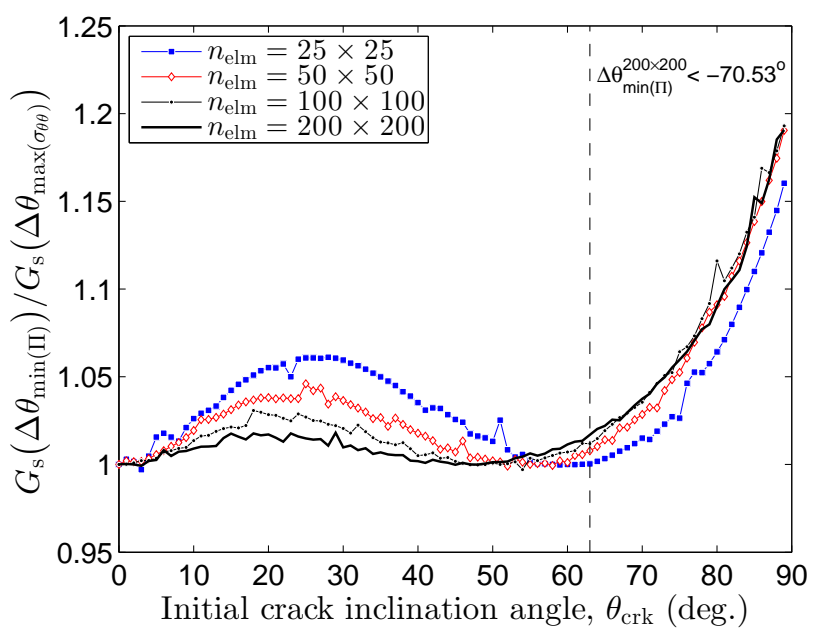

(c) Comparison of the mean energy release rates, as obtained by the minimum energy criterion relative to the maximum hoop stress criterion. It is found that for large crack inclinations $\theta_{\text {crk }}>60^{\circ}$ (i.e. significant mode-II loading) the minimum energy based criterion converges to a crack tip kink direction that exceeds the theoretical limit of the stress-based solution $\left|\Delta \theta_{\max \left(\sigma_{\theta \theta}\right)}\right|<70.53^{\circ}$.

Figure 20: Comparison of crack growth criteria for test case \#2 of a simply supported square plate with an inclined centre crack subjected to a uniform vertical tension. Depending on the crack orientation, the uniaxial tension causes mixed-mode crack tip loading conditions. The mode mixity can range for pure mode-I (for a horizontal crack) to predominantly mode-II (for a nearly vertical crack). 

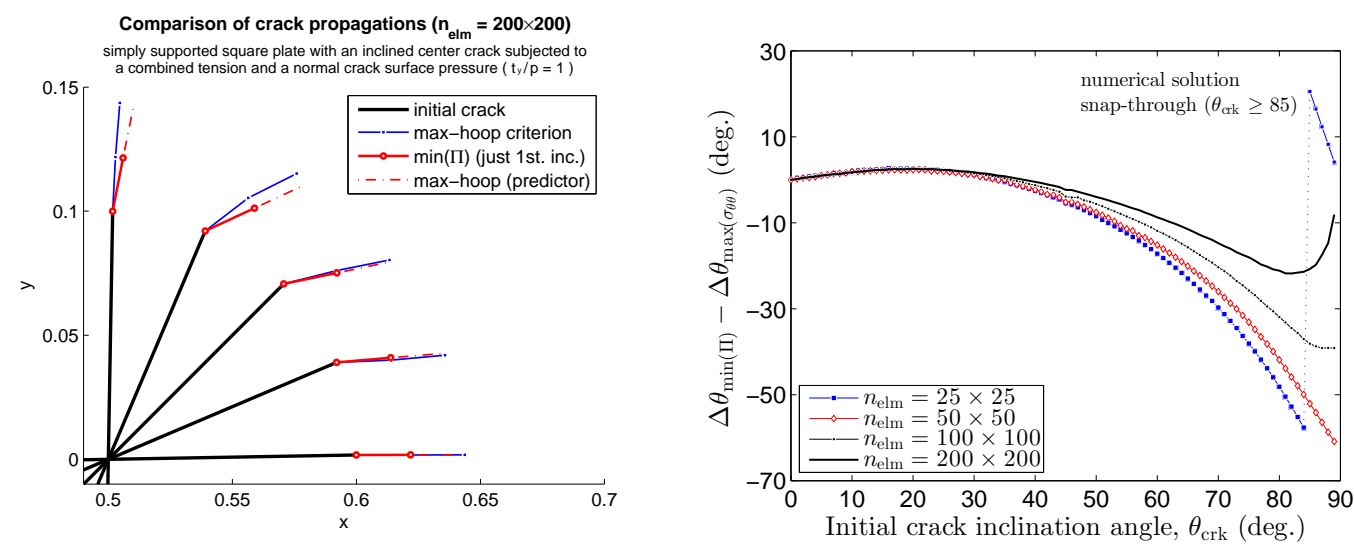

(a) Comparison of incipient crack kink angles

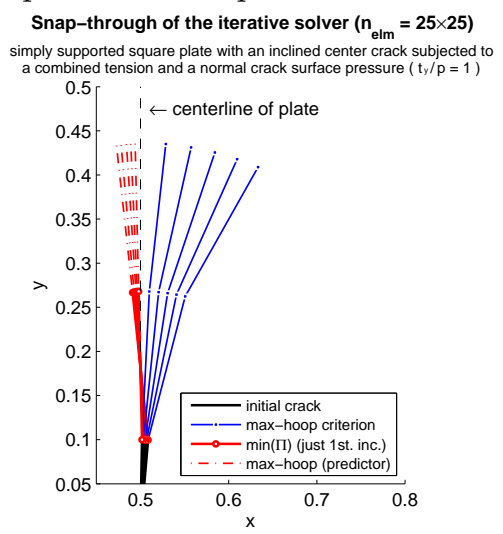

(b) Differences in incipient crack kink angles.

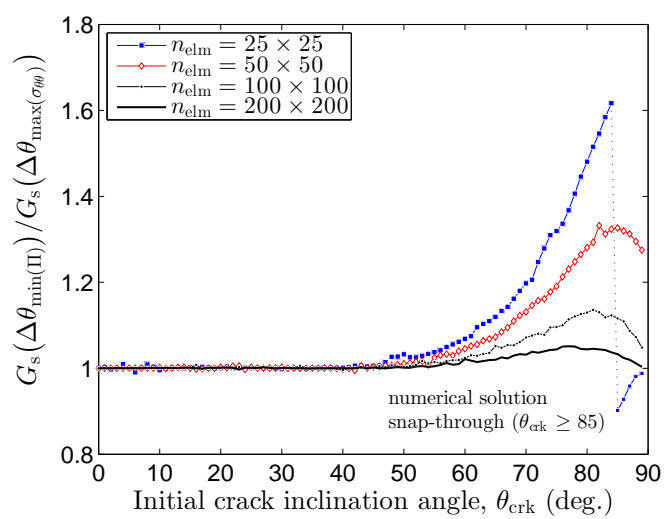

(c) Example of a snap-through of the iterated (d) Comparison of the mean energy release solution and convergence to a local minimiser. rates, as obtained by the minimum energy criterion relative to the maximum hoop stress criterion. Note that for a large crack inclination $\theta_{\text {crk }} \geq 85^{\circ}$ (i.e. for significant mode-II loading) the iterated solution undergoes a snap-through.

Figure 21: Comparison of crack growth criteria for test case \#3 of a simply supported square plate with an inclined centre crack subjected to a uniform vertical tension load and to an equal internal crack surface pressure. Depending on the crack orientation, the uniaxial tension induces a mode-II loading component at the tip of the crack. Interestingly, it is found that for the coarsest discretisation (e.g. for a $25 \times 25$ mesh) and when the initial crack orientation is close to vertical (e.g. $\theta_{\text {crk }} \geq 85$ ) the iterated solution for the crack tip kink angle converges to a sub-optimal solution by snapping to left of the vertical centre line (refer to Figure 21c for a close-up). However, for finer discretisations, the solution converges to the globally optimal solution. 


\subsection{Conclusion}

The XFEM implementation of the algebraic differentiation of the potential energy was shown to be sufficiently accurate for obtaining $G_{\theta}$ and $\mathrm{d} G_{\theta} / \mathrm{d} \theta$. In contrast, the finite differenced solution was oscillatory (i.e. mesh dependent) and, therefore, less accurate. In the test cases involving the plate with an edge crack (refer to Figure 12), minimum potential energy was attained at the crack extension angle $\theta_{\text {inc }}=0$. This satisfied the condition of a vanishing rotational energy release rate, $G_{\theta}=0$, as confirmed by Figures 13 and 15. Subsequently, the principle of vanishing rotational energy release rate was applied in a few more benchmark problems as a criterion for determining the crack growth directions under mixed-mode loading conditions. The solutions were shown to yield higher mean energy dissipation rates than the maximum hoop stress criterion. Note that the maximum hoop stress criterion [11] is a kind of a heuristic since it is inconsistent with Griffith's theory of brittle fracture; nonetheless, it usually serves as a close approximation for predominately mode-I driven crack growth. The seminal 2004 paper [5] dealing with multi-crack growth within XFEM used the maximum stress criterion. This is one of the key differences between our approach and theirs.

\section{Summary}

Three methods have been described for solving the discrete fracture growth problem. The methods were based on: crack length-control, load-control, and on the energygradient. Each method's application was tailored to the type of stability of the fracture configuration at hand. The first method, crack length-control, was aimed at fracture problems where the fracture front was stable. For the method to work the energy function was required to be convex in the solution space defined by a fixed unit of total fracture advance. The method could be used to solve stable or unstable crack growth problems provided the fracture front were stable. The second strategy, external loadcontrol, was limited to fracture problems that involved stable crack growth and a stable fracture front configuration, i.e. the total energy function had to be convex. In this case, the fracture solution could be captured by incrementing the load parameter at each timestep and growing the crack tips whose energy release rates were above the critical value of the material. The third solution strategy relied on the gradient of the energy function to determine the change in the fracture extensions that would give the greatest rate of decrease in the total energy function. The method was shown to be robust for solving non-competing crack growth problems in cases of stable or unstable fracture fronts and stable or unstable fracture growths. The main downside of the method was the lack of robustness in solving competing crack growth at the instance of an unstable fracture front since the converged solution would inherently depended on the initial solution.

The paper focused on the verification of the gradient-descent solution approach in solving general fracture growth problems within a discrete framework of fixed-length crack tip 
incrementation. The biggest concern was the unsatisfactory performance of the proposed gradient-descent solution approach in the particular case of competing crack growth and an unstable fracture front configuration. As a multi-trial solution approach could not be attempted within the discrete framework, an alternative solution strategy was put forth, the so-called offline multi-trial energy minimisation. The offline approach consisted of locally approximated the governing energy function by a quadratic form; then, minimising this energy function with respect to the crack tip extension lengths using multiple trial attempts; finally, coarsening the crack tip extension lengths to fit the discrete framework. The satisfactory performance of the offline approach was verified against the case of competing crack growth and an unstable fracture front.

The computational strategy for determining the fracture energy release rates using the stiffness derivative approach within the extended finite elemet method was described. Good accuracy of the algebraically computed rotational energy release rates was obtained. The minimum energy criterion was then applied in several benchmark problems to determine the crack growth directions under mixed-mode loading conditions. The minimum energy solution normally dissipated more energy than the maximum hoop stress solution; interestingly, the energy criterion also gave rise to crack kink angles greater than $90^{\circ}$ in some cases, whereas the stress criterion caused the crack to kink a few times before the crack aligned to the energetically more favourable direction.

In Part-III of this three-part paper, the computer implementation of the complete solution algorithm is described. A number of multi-crack growth benchmark problems are solved to demonstrate the accuracy and robustness of the implementation, particularly in cases of crack coalescence. The fracture paths and their convergence rates as obtained by the minimum energy approach and the classic maximum hoop stress criterion are compared. Finally, a numerical improvement to the crack growth direction criterion is proposed that offers significant improvements in accuracy and convergence rate of the fracture paths, especially on coarse discretisations.

\section{Supplementary material}

The open-source code XFEM_Fracture2D and supporting material can be found here:

- XFEM_Fracture2D: https://figshare.com/s/0b4394e8fab7191d2692

- competing cracks: https://figshare.com/s/4a7dd5fb0a8634c9fae4

- demo screenshots: https://figshare.com/s/6397737c78beb59f3b58

- demo movies: https://figshare.com/s/73d7b50a7729070c2173 


\section{A Extended finite element implementation}

\section{A.1 XFEM approximation}

The corrected XFEM approximation [13, 12] reads:

$$
\begin{aligned}
\mathbf{u}^{h}(\mathbf{x})= & \sum_{I \in \mathcal{N}_{u}} N_{I}(\mathbf{x}) \mathbf{u}_{I}+\sum_{i=1}^{n_{\text {crk }}} \sum_{I \in \mathcal{N}_{a}^{i}} N_{I}(\mathbf{x})\left(H^{i}(\mathbf{x})-H^{i}\left(\mathbf{x}_{I}\right)\right) \mathbf{a}_{I}^{i} \\
& +\sum_{i=1}^{n_{\text {tip }}} \sum_{l=1}^{4} \sum_{I \in \mathcal{N}_{b}^{i}} N_{I}(\mathbf{x})\left(B^{i, l}(\mathbf{x})-B^{i, l}\left(\mathbf{x}_{I}\right)\right) R^{i}(\mathbf{x}) \mathbf{b}_{I}^{i, l},
\end{aligned}
$$

where $N_{I}(\mathbf{x})$ are the classic piece-wise continuous finite element interpolation functions and where $\mathcal{N}_{u}, \mathcal{N}_{a}^{i}$ and $\mathcal{N}_{b}^{i}$ denote the nodal sets corresponding, respectively, to the standard degrees of freedom (DOF) $\mathbf{u}_{I}$, the Heaviside enrichment DOF $\mathbf{a}_{I}^{i}$ for cracks $i \in\left\{1,2, \ldots, n_{\mathrm{crk}}\right\}$, and the branch enrichment DOF $\mathbf{b}_{I}^{i, l}$ for crack tips $i \in\left\{1,2, \ldots, n_{\text {tip }}\right\}$ and branch functions $l \in\{1,2,3,4\}$. The branch functions are the singular near-tip asymptotic functions characterising the local displacements close to the crack tip [34]; the basis functions are given in terms of polar coordinates $(r, \theta)$ centered at the tip:

$$
\boldsymbol{B}(r, \theta)=\left[\sqrt{r} \sin \frac{\theta}{2}, \sqrt{r} \cos \frac{\theta}{2}, \sqrt{r} \sin \frac{\theta}{2} \sin \theta, \sqrt{r} \cos \frac{\theta}{2} \sin \theta\right]
$$

In addition to the branch enrichment, which makes sense only close to the crack tip, a Heaviside (or discontinuous) enrichment is used to introduce a jump in the displacement field at a general location along a crack [4]. The Heaviside function $H(\mathbf{x})$ is +1 if $\mathbf{x} \in \Omega$ lies on one side of the fracture surface and -1 otherwise. As such, the Heaviside can be defined as the sign of the signed-distance function:

$$
H(\mathbf{x})= \begin{cases}+1 & \text { if }\left(\mathbf{x}-\mathbf{x}_{\mathrm{c}}\right) \cdot \mathbf{n}_{\mathrm{c}}^{-} \geq 0 \\ -1 & \text { otherwise }\end{cases}
$$

where $\mathbf{n}_{\mathrm{c}}^{-}$is the unit normal to the bottom side of the fracture surface and where $\mathbf{x}_{\mathrm{c}} \in \Gamma_{\mathrm{c}}$ is the point on the fracture surface that is the shortest distance to $\mathbf{x}$.

The jumps in the branch enrichment functions $(76)$ across the fracture interface are:

$$
\llbracket \boldsymbol{B} \rrbracket(r, \theta)=\boldsymbol{B}(r, \pi / 2)-\boldsymbol{B}(r,-\pi / 2)=[2 \sqrt{r}, 0,0,0]
$$

The jump in the Heaviside enrichment function (77) across the fracture interface is: 


$$
\llbracket H \rrbracket=H\left(\mathbf{x}^{+}\right)-H\left(\mathbf{x}^{-}\right)=2
$$

The ramp function $R(\mathbf{x})$ is a scalar function that is used to modify the branch enrichment functions such that they vanish outside the support of $R(\mathbf{x})$ [12. The ramp function $R^{i}(\mathbf{x})$ for the $i$ 'th crack tip can be defined using the finite element shape functions:

$$
R^{i}(\mathbf{x})=\sum_{I \in \mathcal{N}_{R}^{i}} N_{I}(\mathbf{x})
$$

where $\mathcal{N}_{R}^{i}$ is the set of the nodes that fall inside the disk of a chosen radius around the $i$ 'th tip. One way to define the set of nodes $\mathcal{N}_{B}^{i}$, i.e. the nodes where the branch enrichment DOF are introduced, is to consider the elements that are within the support domain of the ramp function; in this case, $\mathcal{N}_{B}^{i}$ consists of all the nodes of this patch of elements. On the other hand, the nodal set $\mathcal{N}_{H}^{i}$, i.e. the nodes where the Heaviside enrichment DOF are introduced, are the nodes whose support is cut by the $i$ 'th crack and whose support does not cover any of the crack's tips. Note that a crack tip is localised only by the branch enrichment. In the current implementation we choose a branch enrichment domain that encompasses a few rings of elements around a crack tip [24, 35, 17]. This helps resolve the crack tip field with better accuracy than with topological enrichment 22 , which usually means enriching the nodes of a single element at the crack tip. On the other hand, the present strategy still preserves a relatively well-conditioned system of equations [2, 1, 17] in comparison to geometric enrichment [22], which typically refers to enriching the nodes of a patch of elements irrespectively of the underlying mesh.

In the corrected XFEM approximation (75) the weighted branch enrichment allows the patch of enriched elements to have all of their nodes enriched; consequently, the classic notion of blending elements whose some (but not all) nodes are enriched does not apply. Nonetheless, we loosely refer to the branch enriched elements that have all of their nodes enriched but on which the weight function transitions to zero as blending elements. Similarly, the nodes where the weight function vanishes are called the blending nodes. Regarding Heaviside enrichment, the classic definition of a blending element applies since a node of an element will not be enriched if the node's support covers the crack tip.

\section{A.2 XFEM equation system}

The discrete system of equations of the linear elastostatics problem can be given as:

$$
\underbrace{\left[\begin{array}{lll}
\mathbf{K}_{u u} & \mathbf{K}_{u a} & \mathbf{K}_{u b} \\
\mathbf{K}_{u a} & \mathbf{K}_{a a} & \mathbf{K}_{a b} \\
\mathbf{K}_{b u} & \mathbf{K}_{b a} & \mathbf{K}_{b b}
\end{array}\right]}_{\mathbf{K}} \underbrace{\left[\begin{array}{c}
\mathbf{u} \\
\mathbf{a} \\
\mathbf{b}
\end{array}\right]}_{\mathbf{q}}=\underbrace{\left[\begin{array}{c}
\mathbf{f}_{u} \\
\mathbf{f}_{a} \\
\mathbf{f}_{b}
\end{array}\right]}_{\mathbf{f}}
$$


where $\mathbf{K}$ is the stiffness matrix, $\mathbf{q}$ is the vector of the generalised DOF, and where $\mathbf{f}$ is the nodal force vector. $\mathbf{q}$ is separated into three vectors containing: the standard DOF $\mathbf{u}$, the Heaviside enriched DOF $\mathbf{a}$ and the branch-enriched DOF $\mathbf{b}$, such that

$$
\begin{array}{rll}
\mathbf{u} & =\left[\ldots, u_{I x}, u_{I y}, \ldots\right]^{\mathrm{T}} & I \in \mathcal{N}_{u} \\
\mathbf{a}=\left[\ldots, a_{I x}^{i}, a_{I y}^{i}, \ldots\right]^{\mathrm{T}} & I \in \mathcal{N}_{a}^{i} & i=1, \ldots, n_{\text {crk }} \\
\mathbf{b}=\left[\ldots, b_{I x}^{i, l}, b_{I y}^{i, l}, \ldots\right]^{\mathrm{T}} & I \in \mathcal{N}_{b}^{i, l} & l=1,2,3,4 \quad i=1, \ldots, n_{\text {tip }}
\end{array}
$$

The sub-matrices $\mathbf{K}_{\alpha \beta}$ in the global stiffness matrix $\mathbf{K}$ are:

$$
\mathbf{K}_{\alpha \beta}=\int_{\Omega} \mathbf{B}_{\alpha}(\mathbf{x})^{\mathrm{T}} \mathbf{C B}_{\beta}(\mathbf{x}) \mathrm{d} V \quad \alpha, \beta=u, a, b
$$

The standard part $\mathbf{f}_{u}$ of the global force vector $\mathbf{f}$ is:

$$
\mathbf{f}_{u}=\int_{\Gamma_{t}} \mathbf{N}_{u}^{\mathrm{T}}(\mathbf{x}) \mathbf{t} \mathrm{d} s
$$

whereas the enriched parts can be given as:

$$
\mathbf{f}_{\alpha}=\int_{\Gamma_{\mathrm{c}}^{-}} \llbracket \mathbf{N}_{\alpha} \rrbracket^{\mathrm{T}}(\mathbf{x}) \mathbf{n}^{-} p \mathrm{~d} s \quad \alpha=a, b
$$

where $\llbracket \cdot \rrbracket=(\cdot)^{+}-(\cdot)^{-}$is the jump in the value of a given function from the bottom $\left(\Gamma_{\mathrm{c}}^{-}\right)$ to the top $\left(\Gamma_{\mathrm{c}}^{+}\right)$surface of a crack and where $p$ is the crack pressure. The displacement matrices $\mathbf{N}_{u}, \mathbf{N}_{a}$ and $\mathbf{N}_{b}$, and the strain matrices $\mathbf{B}_{u}, \mathbf{B}_{a}$ and $\mathbf{B}_{b}$ are defined as:

$$
\begin{array}{lll}
\mathbf{N}_{u}=\left[\ldots, \mathbf{N}_{u I}, \ldots\right] & I \in \mathcal{N}_{u} \\
\mathbf{N}_{a}=\left[\ldots, \mathbf{N}_{a I}^{i}, \ldots\right] & I \in \mathcal{N}_{a}^{i} & i=1, \ldots, n_{\text {crk }} \\
\mathbf{N}_{b}=\left[\ldots, \mathbf{N}_{b I}^{i, l}, \ldots\right] & I \in \mathcal{N}_{b}^{i, l} & l=1,2,3,4 \quad i=1, \ldots, n_{\text {tip }} \\
& \\
\mathbf{B}_{u}=\left[\ldots, \mathbf{B}_{u I}, \ldots\right] & I \in \mathcal{N}_{u} \\
\mathbf{B}_{a}=\left[\ldots, \mathbf{B}_{a I}^{i}, \ldots\right] & I \in \mathcal{N}_{a}^{i} & i=1, \ldots, n_{\text {crk }} \\
\mathbf{B}_{b}=\left[\ldots, \mathbf{B}_{b I}^{i, l}, \ldots\right] & I \in \mathcal{N}_{b}^{i, l} & l=1,2,3,4 \quad i=1, \ldots, n_{\text {tip }}
\end{array}
$$

The elements of $\mathbf{N}_{u I}, \mathbf{N}_{a I}^{i}$ and $\mathbf{N}_{b I}^{i, l}$ are: 


$$
\begin{aligned}
& \mathbf{N}_{u I}=\left[\begin{array}{cc}
N_{I} & 0 \\
0 & N_{I}
\end{array}\right] \\
& \mathbf{N}_{a I}^{i}=\left[\begin{array}{cc}
N_{I}\left(H^{i}-H_{I}^{i}\right) & 0 \\
0 & \left.N_{I}\left(H^{i}-H_{I}^{i}\right)\right)
\end{array}\right] \\
& \mathbf{N}_{b I}^{i, l}=\left[\begin{array}{cc}
N_{I}\left(B^{i, l}-B_{I}^{i, l}\right) & 0 \\
0 & N_{I}\left(B^{i, l}-B_{I}^{i, l}\right)
\end{array}\right]
\end{aligned}
$$

and the elements of $\mathbf{B}_{u I}, \mathbf{B}_{a I}^{i}$ and $\mathbf{B}_{a I}^{i, l}$ are:

$$
\begin{aligned}
\mathbf{B}_{u I}= & {\left[\begin{array}{cc}
N_{I, x} & 0 \\
0 & N_{I, y} \\
N_{I, y} & N_{I, x}
\end{array}\right] } \\
\mathbf{B}_{a I}^{i}= & {\left[\begin{array}{cc}
N_{I, x}\left(H^{i}-H_{I}^{i}\right) & 0 \\
0 & N_{I, y}\left(H^{i}-H_{I}^{i}\right) \\
N_{I, y}\left(H^{i}-H_{I}^{i}\right) & N_{I, x}\left(H^{i}-H_{I}^{i}\right)
\end{array}\right] } \\
\mathbf{B}_{b I}^{i, l}= & {\left[\begin{array}{cc}
\left(N_{I}\left(B^{i, l}-B_{I}^{i, l}\right)\right)_{, x} & 0 \\
0 & \left(N_{I}\left(B^{i, l}-B_{I}^{i, l}\right)\right)_{, y} \\
\left(N_{I}\left(B^{i, l}-B_{I}^{i, l}\right)\right)_{, y} & \left(N_{I}\left(B^{i, l}-B_{I}^{i, l}\right)\right)_{, x}
\end{array}\right] }
\end{aligned}
$$

where the comma-separated subscripts denote partial differentiation, i.e. $f_{, x} \equiv \frac{\partial f}{\partial x}$.

\section{A.3 Element-level derivatives}

Concerning an element that is cut by a crack and that is subjected to a partial rotation (refer to Figure 11 to the yellow element on the crack vertex), the variations of the strain and of the displacement operator matrices, and of the element's Jacobian need to be computed at each integration sub-cell of the element. The first step is to obtain a relationship between the change in the element's local coordinate point $\delta \mathbf{X}^{\mathrm{e}}$ and the change in the element's corresponding material coordinate $\delta \mathbf{x}^{\mathrm{e}}$ as a function of the changes in the element's vertices $\delta \mathbf{x}_{I}^{\mathrm{e}}$ and in the vertices of the element's sub-cells $\delta \mathbf{x}_{J}^{\mathrm{c}}$. The compatibility between the displacements of a material point, as interpolated by the shape functions of the element and the shape functions of a sub-cell, reads:

$$
N_{I}^{\mathrm{e}}\left(\mathbf{X}^{\mathrm{e}}+\delta \mathbf{X}^{\mathrm{e}}\right)\left(\mathbf{x}_{I}^{\mathrm{e}}+\delta \mathbf{x}_{I}^{\mathrm{e}}\right)-N_{I}^{\mathrm{e}}\left(\mathbf{X}^{\mathrm{e}}\right) \mathbf{x}_{I}^{\mathrm{e}}=N_{J}^{\mathrm{c}}\left(\mathbf{X}^{\mathrm{c}}\right) \delta \mathbf{x}_{J}^{\mathrm{c}}
$$


In other words, 100 states that the displacements of two coincidental material points, as interpolated by the element's shape functions and as interpolated by the shape functions of the element's sub-cell need to be the same for any displacement of the element's vertices. In turn, the compatibility equation 100 yields the following relationship:

$$
\begin{gathered}
N_{I}^{\mathrm{e}} \delta \mathbf{x}_{I}^{\mathrm{e}}+\delta \mathbf{X}^{\mathrm{e}} \frac{\mathrm{d} N_{I}^{\mathrm{e}}}{\mathrm{d} \mathbf{X}^{\mathrm{e}}} \mathbf{x}_{I}^{\mathrm{e}}=N_{J}^{\mathrm{c}} \delta \mathbf{x}_{J}^{\mathrm{c}} \\
\delta \mathbf{X}^{\mathrm{e}}=\left(N_{J}^{\mathrm{c}} \delta \mathbf{x}_{J}^{\mathrm{c}}-N_{I}^{\mathrm{e}} \delta \mathbf{x}_{I}^{\mathrm{e}}\right)\left(\frac{\mathrm{d} N_{I}^{\mathrm{e}}}{\mathrm{d} \mathbf{X}^{\mathrm{e}}} \mathbf{x}_{I}^{\mathrm{e}}\right)^{-1}
\end{gathered}
$$

The displacement of an interior point due to the displacement of the elements nodes is $\delta \mathbf{x}^{\mathrm{e}}=N_{I}^{\mathrm{e}} \delta \mathbf{x}_{I}^{\mathrm{e}}$ whereas the displacement of the same point, as interpolated by the shapes of the element's sub-cell, is $\delta \mathbf{x}^{\mathrm{c}}=N_{I}^{\mathrm{c}} \delta \mathbf{x}_{I}^{\mathrm{c}}$. Generally, these two displacements do not coincide, i.e. $\delta \mathbf{x}^{\mathrm{e}} \neq \delta \mathbf{x}^{\mathrm{c}}$, and so consistency is enforced by $\delta \mathbf{X}^{\mathrm{e}}$, as determined by (102). The variations $\delta \mathbf{X}^{\mathrm{e}}$ and likewise $\delta^{2} \mathbf{X}^{\mathrm{e}}$ will not be zero if the displacement mismatch variations $\delta \overline{\mathbf{x}}$ and $\delta^{2} \overline{\mathbf{x}}$ are not zero; the latter variations can be defined as follows:

$$
\begin{aligned}
\delta \overline{\mathbf{x}} & =N_{J}^{\mathrm{c}} \delta \mathbf{x}_{J}^{\mathrm{c}}-N_{I}^{\mathrm{e}} \delta \mathbf{x}_{I}^{\mathrm{e}} \\
\delta^{2} \overline{\mathbf{x}} & =\left(N_{J}^{\mathrm{c}} \delta^{2} \mathbf{x}_{J}^{\mathrm{c}}+\delta N_{J}^{\mathrm{c}} \delta \mathbf{x}_{J}^{\mathrm{c}}\right)-\left(N_{I}^{\mathrm{e}} \delta^{2} \mathbf{x}_{I}^{\mathrm{e}}+\delta N_{I}^{\mathrm{e}} \delta \mathbf{x}_{I}^{\mathrm{e}}\right)
\end{aligned}
$$

$\delta \overline{\mathbf{x}}$ and $\delta^{2} \overline{\mathbf{x}}$ can then be used to determine $\delta \mathbf{X}^{\mathrm{e}}$ and $\delta^{2} \mathbf{X}^{\mathrm{e}}$ :

$$
\begin{aligned}
\delta \mathbf{X}^{\mathrm{e}} & =\delta \overline{\mathbf{x}} \mathbf{J}^{-1} \\
\delta^{2} \mathbf{X}^{\mathrm{e}} & =\delta^{2} \overline{\mathbf{x}} \mathbf{J}^{-1}+\delta \overline{\mathbf{x}} \delta\left(\mathbf{J}^{-1}\right)
\end{aligned}
$$

The variations of the element shape functions $\delta N_{I}^{\mathrm{e}}$ and $\delta^{2} N_{I}^{\mathrm{e}}$ can be determined easily since $\delta \mathbf{X}^{\mathrm{e}}$ and $\delta^{2} \mathbf{X}^{\mathrm{e}}$ are known from (105) and (106). The enriched shape function jump variations $\delta \llbracket N_{I} \rrbracket$ and $\delta^{2} \llbracket N_{I} \rrbracket$ along a crack are only due to the variations of the shape functions $N_{I}^{\mathrm{e}}$ that multiply the enrichment function jumps; however, the enrichment function jumps are otherwise constant with respect to the rotation of a crack branch. The variations of the enriched shape functions are zero if the element undergoes a uniform translation or a rotation and not zero if an element undergoes a distortion. In the latter case, the variations are generally not zero because the translation of some of the elements nodes causes a change in the values of standard shape functions $N_{I}^{\mathrm{e}}$. Thus, for an element in partial rotation, e.g. the element that contains the crack-kink (refer to Figure 11), the variations of the enriched shape functions $\delta \llbracket N_{\psi I} \rrbracket$ and $\delta^{2} \llbracket N_{\psi I} \rrbracket$ are obtained as:

$$
\begin{aligned}
\delta \llbracket N_{\psi I} \rrbracket & =\quad \delta N_{I} \llbracket \psi \rrbracket, \\
\delta^{2} \llbracket N_{\psi I} \rrbracket & =\delta^{2} N_{I} \llbracket \psi \rrbracket,
\end{aligned}
$$


where $\psi$ refers to either the Heaviside or the branch enrichment. The variations of the terms in equations (65), (66), (69) and (70) are detailed below: (note that the vector derivatives are assumed to be: $\partial / \partial \mathbf{X}=[\partial / \partial \xi \partial / \partial \eta]^{\mathrm{T}}$, and $\left.\partial / \partial \mathbf{x}=[\partial / \partial x \partial / \partial y]^{\mathrm{T}}\right)$

$$
\begin{aligned}
\delta \mathbf{J} & =\frac{\partial N_{I}}{\partial \mathbf{X}} \delta \mathbf{x}_{I}+\delta\left(\frac{\partial N_{I}}{\partial \mathbf{X}}\right) \mathbf{x}_{I} \\
\delta^{2} \mathbf{J} & =\frac{\partial N_{I}}{\partial \mathbf{X}} \delta^{2} \mathbf{x}_{I}+2 \delta\left(\frac{\partial N_{I}}{\partial \mathbf{X}}\right) \delta \mathbf{x}_{I}+\delta^{2}\left(\frac{\partial N_{I}}{\partial \mathbf{X}}\right) \mathbf{x}_{I}
\end{aligned}
$$

The determinant of the Jacobian and its variations are given as:

$$
\begin{aligned}
\operatorname{det}(\mathbf{J})=\left(\frac{\partial N_{I}}{\partial \xi} \frac{\partial N_{J}}{\partial \eta}-\frac{\partial N_{I}}{\partial \eta} \frac{\partial N_{J}}{\partial \xi}\right) x_{I} y_{J} \\
\delta \operatorname{det}(\mathbf{J})=\left(\frac{\partial N_{I}}{\partial \xi} \frac{\partial N_{J}}{\partial \eta}-\frac{\partial N_{I}}{\partial \eta} \frac{\partial N_{J}}{\partial \xi}\right) \delta x_{I} y_{J}+\delta \xi \frac{\partial}{\partial \xi}\left(\frac{\partial N_{I}}{\partial \xi} \frac{\partial N_{J}}{\partial \eta}-\frac{\partial N_{I}}{\partial \eta} \frac{\partial N_{J}}{\partial \xi}\right) x_{I} y_{J} \\
+\left(\frac{\partial N_{I}}{\partial \xi} \frac{\partial N_{J}}{\partial \eta}-\frac{\partial N_{I}}{\partial \eta} \frac{\partial N_{J}}{\partial \xi}\right) x_{I} \delta y_{J}+\delta \eta \frac{\partial}{\partial \eta}\left(\frac{\partial N_{I}}{\partial \eta} \frac{\partial N_{J}}{\partial \xi}-\frac{\partial N_{I}}{\partial \eta} \frac{\partial N_{J}}{\partial \xi}\right) x_{I} y_{J} \\
\delta^{2} \operatorname{det}(\mathbf{J})=\left(\frac{\partial N_{I}}{\partial \xi} \frac{\partial N_{J}}{\partial \eta}-\frac{\partial N_{I}}{\partial \eta} \frac{\partial N_{J}}{\partial \xi}\right) \delta^{2} x_{I} y_{J}+\delta \xi^{2} \frac{\partial^{2}}{\partial \xi^{2}}\left(\frac{\partial N_{I}}{\partial \xi} \frac{\partial N_{J}}{\partial \eta}-\frac{\partial N_{I}}{\partial \eta} \frac{\partial N_{J}}{\partial \xi}\right) x_{I} y_{J} \\
+\left(\frac{\partial N_{I}}{\partial \xi} \frac{\partial N_{J}}{\partial \eta}-\frac{\partial N_{I}}{\partial \eta} \frac{\partial N_{J}}{\partial \xi}\right) 2 \delta x_{I} \delta y_{J}+2 \delta \xi \delta \eta \frac{\partial^{2}}{\partial \xi \partial \eta}\left(\frac{\partial N_{I}}{\partial \eta} \frac{\partial N_{J}}{\partial \xi}-\frac{\partial N_{I}}{\partial \eta} \frac{\partial N_{J}}{\partial \xi}\right) x_{I} y_{J} \\
+\left(\frac{\partial N_{I}}{\partial \xi} \frac{\partial N_{J}}{\partial \eta}-\frac{\partial N_{I}}{\partial \eta} \frac{\partial N_{J}}{\partial \xi}\right) x_{I} \delta^{2} y_{J}+\delta \eta^{2} \frac{\partial^{2}}{\partial \eta^{2}}\left(\frac{\partial N_{I}}{\partial \eta} \frac{\partial N_{J}}{\partial \xi}-\frac{\partial N_{I}}{\partial \eta} \frac{\partial N_{J}}{\partial \xi}\right) x_{I} y_{J}
\end{aligned}
$$

The first and second differentials of the inverse of the Jacobian are: (note that $\mathbf{J}^{-1} \mathbf{J}=\mathbf{I}$ )

$$
\begin{aligned}
\delta \mathbf{J}^{-1} & =-\mathbf{J}^{-1} \delta \mathbf{J} \mathbf{J}^{-1} \\
\delta^{2} \mathbf{J}^{-1} & =-\mathbf{J}^{-1} \delta^{2} \mathbf{J ~ J ~ J}^{-1}+2 \mathbf{J}^{-1} \delta \mathbf{J} \mathbf{J}^{-1} \delta \mathbf{J} \mathbf{J}^{-1}
\end{aligned}
$$

The Cartesian derivatives of the shape functions of the elements that are subjected to partial rotations and that are cut by a crack (i.e. use sub-cell integration), read:

$$
\begin{gathered}
\delta \frac{\partial N_{I}}{\partial \mathbf{x}}=\delta \mathbf{J}^{-1} \frac{\partial N_{I}}{\partial \mathbf{X}}+\mathbf{J}^{-1} \delta \frac{\partial N_{I}}{\partial \mathbf{X}} \\
\delta^{2} \frac{\partial N_{I}}{\partial \mathbf{x}}=\delta^{2} \mathbf{J}^{-1} \frac{\partial N_{I}}{\partial \mathbf{X}}+\mathbf{J}^{-1} \delta^{2} \frac{\partial N_{I}}{\partial \mathbf{X}}
\end{gathered}
$$


On the other hand, the shape function derivatives of the elements undergoing partial rotations but that are not cut by a crack (e.g. the white elements in Figure 11) read:

$$
\begin{aligned}
\delta \frac{\partial N_{I}}{\partial \mathbf{x}} & =\delta \mathbf{J}^{-1} \frac{\partial N_{I}}{\partial \mathbf{X}} \\
\delta^{2} \frac{\partial N_{I}}{\partial \mathbf{x}} & =\delta^{2} \mathbf{J}^{-1} \frac{\partial N_{I}}{\partial \mathbf{X}}
\end{aligned}
$$

Finally, the variations of the strain-like matrix read:

$$
\begin{aligned}
\delta \mathbf{B}_{I} & =\left[\begin{array}{ccc}
\delta \frac{\partial N_{I}}{\partial x} & 0 & \delta \frac{\partial N_{I}}{\partial y} \\
0 & \delta \frac{\partial N_{I}}{\partial y} & \delta \frac{\partial N_{I}}{\partial x}
\end{array}\right] \\
\delta^{2} \mathbf{B}_{I} & =\left[\begin{array}{ccc}
\delta^{2} \frac{\partial N_{I}}{\partial x} & 0 & \delta^{2} \frac{\partial N_{I}}{\partial y} \\
0 & \delta^{2} \frac{\partial N_{I}}{\partial y} & \delta^{2} \frac{\partial N_{I}}{\partial x}
\end{array}\right]
\end{aligned}
$$

whereas the displacement jump like matrix is defined as:

$$
\llbracket \mathbf{N} \rrbracket_{\psi I}=\left[\begin{array}{cc}
N_{I} \llbracket \psi \rrbracket & 0 \\
0 & N_{I} \llbracket \psi \rrbracket
\end{array}\right]
$$

where $\psi$ refers to any of the enrichment functions, i.e. branch (76) or Heaviside (77). Considering the rotation of a crack tip extension (see Figure 11) the position of a point $\mathbf{x}=(x, y)$ in the Cartesian coordinate system can be expressed in terms of the polar coordinates; then, a change in $\mathbf{x}$ is a function of the change in the polar angle $\theta$ :

$$
\begin{aligned}
\delta_{i} x & =-y \delta \theta_{i}, & \delta_{i} y & =x \delta \theta_{i} \\
\delta_{i}^{2} x & =-x \delta \theta_{i}, & \delta_{i}^{2} y & =-y \delta \theta_{i}
\end{aligned}
$$




\section{References}

[1] K. Agathos, E. Chatzi, and S. P. A. Bordas. "Stable 3D extended finite elements with higher order enrichment for accurate non planar fracture". In: Computer Methods in Applied Mechanics and Engineering 306.October 2015 (2016), pp. 1946. ISSN: 00457825.

[2] K. Agathos et al. "A well-conditioned and optimally convergent XFEM for 3D linear elastic fracture". In: International Journal for Numerical Methods in Engineering 105.9 (2016), pp. 643-677. ISSN: 00295981.

[3] M. P. Areias and T. Belytschko. "Analysis of three-dimensional crack initiation and propagation using the extended finite element method". In: International Journal for Numerical Methods in Engineering 63.5 (2005), pp. 760-788. ISSN: 0029-5981.

[4] T. Belytschko et al. "Arbitrary discontinuities in finite elements". In: International Journal for Numerical Methods in Engineering 50.4 (2001), pp. 993-1013. ISSN: 0029-5981.

[5] E. Budyn et al. "A method for multiple crack growth in brittle materials without remeshing". In: International Journal for Numerical Methods in Engineering 61.10 (2004), pp. 1741-1770. ISSN: 0029-5981.

[6] A. Chambolle, G. A. Francfort, and J. J. Marigo. "When and how do cracks propagate?" In: Journal of the Mechanics and Physics of Solids 57.9 (2009), pp. 16141622. ISSN: 00225096.

[7] R. J. Charles. "Static fatigue of glass. I". In: Journal of Applied Physics 29.11 (1958), pp. 1554-1560. ISSN: 00218979.

[8] R. J. Charles. "Static Fatigue of Glass. II". In: Journal of Applied Physics 29.11 (1958), p. 1554. ISSN: 00218979.

[9] B. R. Davis, P. A. Wawrzynek, and A. R. Ingraffea. "3-D simulation of arbitrary crack growth using an energy-based formulation - Part I: Planar growth". In: Engineering Fracture Mechanics 115 (2014), pp. 204-220. ISSN: 00137944.

[10] B. R. Davis, P. A. Wawrzynek, and A. R. Ingraffea. "Simulation of Arbitrary Mixed-Mode Crack Growth Using an Energy-Based Approach". In: ed. by J. Carroll and S. Daly. Cham: Springer International Publishing, 2015. Chap. Simulation, pp. 1-9. ISBN: 978-3-319-06977-7.

[11] F. Erdogan and G. C. Sih. "On the Crack Extension in Plates Under Plane Loading and Transverse Shear". In: Journal of Basic Engineering 85.4 (1963), p. 519. ISSN: 00219223.

[12] T. P. Fries. "Overview and comparison of different variants of the XFEM". In: PAMM 14.1 (2014), pp. 27-30. ISSN: 16177061.

[13] T. P. Fries and T. Belytschko. "The extended/generalized finite element method: An overview of the method and its applications". In: International Journal for Numerical Methods in Engineering (2010), n/a-n/a. ISSN: 00295981.

[14] R. V. Gol'dstein and R. L. Salganik. "Brittle fracture of solids with arbitrary cracks". In: International Journal of Fracture 10.4 (1974), pp. 507-523. ISSN: 03769429 . 
[15] A. A. Griffith. "The Phenomena of Rupture and Flow in Solids". In: Philosophical Transactions of the Royal Society of London A221 (1920), pp. 163-198.

[16] A. A. Griffith. "The Phenomena of Rupture and Flow in Solids". In: Philosophical Transactions of the Royal Society of London. Series A, Containing Papers of a Mathematical or Physical Character 221 (1921), pp. 163-198.

[17] P. Gupta and C. A. Duarte. "Coupled formulation and algorithms for the simulation of non-planar three-dimensional hydraulic fractures using the generalized finite element method". In: International Journal for Numerical and Analytical Methods in Geomechanics 40.10 (2016), pp. 1402-1437. ISSN: 03639061. arXiv: nag.2347 [10.1002].

[18] K. Hayashi and S. Nemat-Nasser. "Energy-Release Rate and Crack Kinking Under Combined Loading". In: Journal of Applied Mechanics 48.3 (1981), p. 520. ISSN: 00218936 .

[19] M. A. Hussain, S. L. Pu, and J. Underwood. "Strain Energy Release Rate for a Crack Under Combined Mode I and Mode II". In: Fracture analysis 560 (1974), pp. $2-28$.

[20] C. G. Hwang et al. "On the virtual crack extension method for calculation of the rates of energy release rate". In: Engineering Fracture ... 59.4 (1998), pp. 521-542.

[21] B. L. Karihaloo, L. M. Keer, and S. Nemat-Nasser. "Crack kinking under nonsymmetric loading". In: Engineering Fracture Mechanics 13.4 (1980), pp. 879-888. ISSN: 0013-7944.

[22] P. Laborde et al. "High-order extended finite element method for cracked domains". In: International Journal for Numerical Methods in Engineering 64.3 (2005), pp. 354-381. ISSN: 0029-5981.

[23] S. C. Lin and J. F. Abel. "Variational approach for a new direct-integration form of the virtual crack extension method". In: International Journal of Fracture 235.3 (1988), pp. 217-235.

[24] X. Y. Liu, Q. Z. Xiao, and B. L. Karihaloo. "XFEM for direct evaluation of mixed mode SIFs in homogeneous and bi-materials". In: International Journal for $\mathrm{Nu}$ merical Methods in Engineering 59.8 (2004), pp. 1103-1118. ISSN: 0029-5981.

[25] E. Orowan. "Fracture and strength of solids". In: Reports on Progress in Physics 12.1 (1949), p. 309. ISSN: 00344885.

[26] D. M. Parks. "A stiffness derivative finite element technique for determination of crack tip stress intensity factors". In: International Journal of Fracture 10.4 (1974), pp. 487-502. ISSN: 0376-9429.

[27] T. Rabczuk, S. Bordas, and G. Zi. "A three-dimensional meshfree method for continuous multiple-crack initiation, propagation and junction in statics and dynamics". In: Computational Mechanics 40.3 (2007), pp. 473-495. ISSN: 0178-7675.

[28] A. Salvadori. "Crack kinking in brittle materials". In: Journal of the Mechanics and Physics of Solids 58.11 (2010), pp. 1835-1846. ISSN: 00225096.

[29] A. Salvadori. "A plasticity framework for (linear elastic) fracture mechanics". In: Journal of the Mechanics and Physics of Solids 56.5 (2008), pp. 2092-2116. ISSN: 00225096 . 
[30] G. C. Sih. "Strain-energy-density factor applied to mixed mode crack problems". In: International Journal of fracture 10.3 (1974), pp. 305-321.

[31] I. N. Sneddon. "The Distribution of Stress in the Neighbourhood of a Crack in an Elastic Solid". In: Proceedings of the Royal Society A: Mathematical, Physical and Engineering Sciences 187.1009 (1946), pp. 229-260. ISSN: 1364-5021.

[32] S. M. Wiederhorn and L. H. Bolz. "Stress Corrosion and Static Fatigue of Glass". In: Journal of the American Ceramic Society 53.10 (1970), pp. 543-548. ISSN: 1551-2916.

[33] J. G. Williams. "On the calculation of energy release rates for cracked laminates". In: International Journal of Fracture 36.2 (1988), pp. 101-119. ISSN: 0376-9429.

[34] M. Williams. "On the Stress Distribution at the Base of a Stationary Crack". In: ASME Journal of applied mechanics 24.109 (1957), p. 114.

[35] Q. Z. Xiao and B. L. Karihaloo. "Improving the accuracy of XFEM crack tip fields using higher order quadrature and statically admissible stress recovery". In: International Journal for Numerical Methods in Engineering 66.9 (2006), pp. 13781410. ISSN: 0029-5981. 